\title{
Elasticity, fluctuations, and vortex pinning in ferromagnetic superconductors: A columnar elastic glass
}

\author{
A. M. Ettouhami, ${ }^{1}$ Karl Saunders, ${ }^{2}$ L. Radzihovsky, ${ }^{3}$ and John Toner ${ }^{4}$ \\ ${ }^{1}$ Department of Physics, University of Florida, P.O. Box 118440, Gainesville, Florida 32611-8440, USA \\ ${ }^{2}$ Department of Physics, California Polytechnic State University, San Luis Obispo, California 93407, USA \\ ${ }^{3}$ Department of Physics, University of Colorado, 390UCB, Boulder, Colorado 80309-0390, USA \\ ${ }^{4}$ Department of Physics, Materials Science Institute and Institute of Theoretical Science, University of Oregon, \\ Eugene, Oregon 97403, USA
}

(Received 21 December 2004; published 17 June 2005)

\begin{abstract}
We study the elasticity, fluctuations, and pinning of a putative spontaneous vortex solid in ferromagnetic superconductors. Using a rigorous thermodynamic argument, we show that in the idealized case of vanishing crystalline pinning anisotropy the long-wavelength tilt modulus of such a vortex solid vanishes identically, as guaranteed by the underlying rotational invariance. The vanishing of the tilt modulus means that, to lowest order, the associated tension elasticity is replaced by the softer, curvature elasticity. The effect of this is to make the spontaneous vortex solid qualitatively more susceptible to the disordering effects of thermal fluctuations and random pinning. We study these effects, taking into account the nonlinear elasticity, that, in three dimensions, is important at sufficiently long length scales, and showing that a "columnar elastic glass" phase of vortices results. This phase is controlled by a previously unstudied zero-temperature fixed point, and it is characterized by elastic moduli that have universal strong wave-vector dependence out to arbitrarily long length scales, leading to non-Hookean elasticity. We argue that, although translationally disordered for weak disorder, the columnar elastic glass is stable against the proliferation of dislocations and is, therefore, a topologically ordered elastic glass. As a result, the phenomenology of the spontaneous vortex state of isotropic magnetic superconductors differs qualitatively from a conventional, external-field-induced mixed state. For example, for weak external fields $H$, the magnetic induction scales universally like $B(H) \sim B(0)+c H^{\alpha}$, with $\alpha \approx 0.72$.
\end{abstract}

DOI: $10.1103 / P h y s R e v B .71 .224506$

PACS number(s): 74.25.Qt, 74.25.Ha, 74.40.+k

\section{INTRODUCTION}

It has been more than 20 years since superconducting materials containing a periodic arrangement of magnetic rareearth ions were discovered. ${ }^{1}$ These included the rare-earth compounds $R \mathrm{Rh}_{4} \mathrm{~B}_{4}, \mathrm{R}_{x} \mathrm{Mo}_{6} \mathrm{~S}_{8}$, and $\mathrm{R}_{x} \mathrm{Mo}_{6} \mathrm{Se}_{8}$, with $x=1.0$ or 1.2 and $R$ a rare-earth material (such as Lu, Y, Tm, Er, Ho, and Dy). As temperature was varied, these materials displayed either a superconducting or a magnetically ordered phase, but no phase where both superconductivity and magnetism coexisted simultaneously. More recent efforts, driven by the need to understand high- $T_{c}$ superconductivity and other strongly correlated materials, have led to experimental discoveries of systems exhibiting coexistence of superconductivity and magnetic order, such as the rare-earth nickel borocarbides ${ }^{2} R \mathrm{Ni}_{2} \mathrm{~B}_{2} \mathrm{C}$ with relatively high superconducting transition temperatures $\left(T_{c} \simeq 16.5 \mathrm{~K}\right.$ for the $\mathrm{Lu}$ and $15 \mathrm{~K}$ for the $Y$ compound). These materials exhibit a rich phase diagram that includes superconductivity, antiferromagnetism, ferromagnetism, and spiral magnetic order. ${ }^{3,4}$ Although most of these materials are antiferromagnets, there is now ample experimental evidence ${ }^{5}$ that below both the Curie $(\simeq 2.3 \mathrm{~K})$ and the superconducting transition temperatures $(\simeq 10.5 \mathrm{~K})$, superconductivity and ferromagnetism competitively coexist in $\mathrm{ErNi}_{2} \mathrm{~B}_{2} \mathrm{C}$ compounds. Other possible examples of ferromagnetic superconductors (FS) are the recently discovered high-temperature superconductor $\mathrm{Sr}_{2} \mathrm{YRu}_{1-x} \mathrm{Cu}_{x} \mathrm{O}_{6}$ and the putative $p$-wave triplet strontium ruthenate superconductor, $\mathrm{Sr}_{2} \mathrm{RuO}_{4}$, which spontaneously breaks time-reversal symmetry, as well as the recently discovered compounds $\mathrm{RuSr}_{2} \mathrm{GdCu}_{2} \mathrm{O}_{8},{ }^{6} \mathrm{UGe}_{2},{ }^{7} \mathrm{ZrZn}_{2},{ }^{8}$ and URhGe. ${ }^{9}$

Although there are many interesting unanswered microscopic questions regarding the nature of such ferromagnetic superconductors, much of their low-energy phenomenology is dictated by general symmetry principles. These constrain the form of the Ginzburg-Landau theory involving the local magnetization $\mathbf{M}$ and the superconducting order parameter $\Psi$, which we expect to describe the low-energy equilibrium thermodynamics. As we discuss in Sec. II, the resulting theory predicts a rich phenomenology, ${ }^{10-16}$ which among other phases admits a very interesting spontaneous vortex (SV) state driven by the spontaneous magnetization, rather than by an external magnetic field $\mathbf{H}$. The argument made in the context of borocarbides ${ }^{15,16}$ (which we believe applies more generally) implies that ferromagnetic superconductors are expected to exhibit such a spontaneous $(H=0)$ vortex state. Recent small-angle neutron-scattering experiments ${ }^{17}$ on $\mathrm{ErNi}_{2}{ }^{11} \mathrm{~B}_{2} \mathrm{C}$ provide some evidence for the existence of a $\mathrm{SV}$ state. The combined effects of soft elasticity, random pinning, and thermal fluctuations lead to a unique phenomenology for the SV solid; this unique phenomenology is the subject of this paper.

We will show, in particular, that for $H=0$ and vanishing crystal anisotropy pinning fields, ${ }^{18}$ the elastic properties of the resulting SV solid differ dramatically and qualitatively 
from those of a conventional Abrikosov lattice. The key underlying difference is the vanishing of the tilt modulus, which is guaranteed by the underlying rotational invariance. ${ }^{18}$ This invariance is, of course, broken by the magnetization $\mathbf{M}$, but because this symmetry breaking is spontaneous, the tilt modulus remains zero. This is reflected by the invariance of the energy under a simultaneous global rotation of the magnetization and vortex lattice induced by it. Formally, the vanishing of the tilt modulus corresponds to the vanishing of the "mass" of the local field, which is a Goldstone mode associated with the rotational symmetry that is spontaneously broken by the vortex lattice and magnetization. This contrasts strongly with a conventional vortex solid, where the rotational symmetry is explicitly broken by the applied field $\mathbf{H}$ and crystalline anisotropy. Of course, in a real sample there will always be some degree of crystalline anisotropy. We will show that our results for the columnar elastic glass phase of the SV solid are valid out to a long length scale that depends on the strength of crystalline anisotropy pinning fields. This length scale diverges with vanishing anisotropy so that our results best describe materials with weak crystalline anisotropy.

All of the conclusions that we draw about other distinctive properties of the SV solid for $\mathbf{H}=\mathbf{0}$ are a direct consequence of this important observation. In particular, we find that this "softness" (i.e., vanishing tilt modulus) of the SV lattice dramatically enhances the effects of quenched disorder and thermal fluctuations. As in conventional vortex lattices, ${ }^{19}$ any amount of disorder $\Delta$, however weak, is sufficient to destroy translational order in SV lattices. Here we find that the Larkin lengths $R_{c}^{\perp}$ and $R_{c}^{z}$, beyond which translational order is destroyed by random forces and torques, are highly anisotropic both in their magnitude and in the way they scale with pinning strength $\Delta$. We predict

$$
\begin{aligned}
& R_{c}^{\perp} \propto 1 / \Delta^{2 / 3}, \\
& R_{c}^{z} \propto 1 / \Delta^{1 / 3},
\end{aligned}
$$

in $d=3$ dimensions, a prediction that can, in principle, be checked in neutron-scattering and transport measurements. However, as we will show, for sufficiently weak pinning (such that dislocation loops remain bound and the elastic description remains valid), the disorder also qualitatively alters the SV solid's long-scale elastic properties, leading to "anomalous elasticity": the universal scaling of elastic moduli with wave vector $\mathbf{q}$ out to arbitrarily long length scales (small $q$ ), with some elastic moduli vanishing and others diverging as the wave vector $\mathbf{q} \rightarrow 0$. Other related long length scale elastic properties include a universal negative Poisson ratio and a non-Hookean elasticity. This anomalous elasticity is completely distinct from the well-known wavevector dependence of the tilt and bulk moduli at short length scales (less than the London penetration length $\lambda$, or, equivalently, at wave vectors $q$ such that $q \lambda \gg 1$ ) in conventional vortex lattices. ${ }^{20}$ This long-wavelength anomalous behavior is characteristic of a kind of topologically ordered columnar elastic glass phase of vortices, which can be shown ${ }^{21}$ to be stable, for weak disorder, against the proliferation of dislocations. This spontaneous vortex solid is another example of

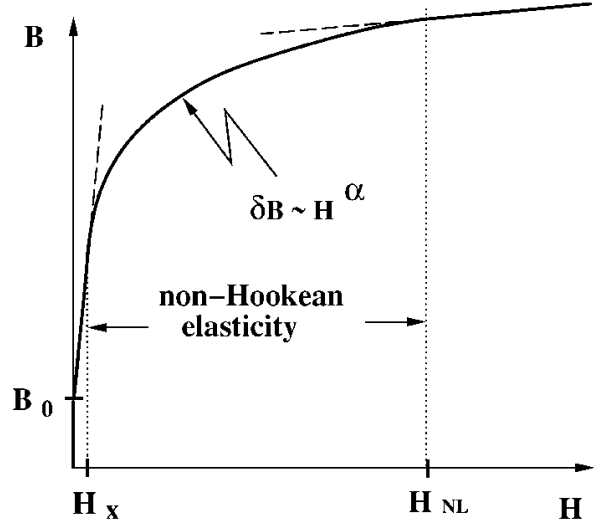

FIG. 1. The nonlinear and universal power-law $B(H)$ scaling, which at weakest fields $H<H_{c r}$ and strongest fields $H>H_{N L}$ is cut off by the crystal symmetry breaking anisotropy and $\xi^{N L}$, respectively.

a class of soft elastic systems, ${ }^{22}$ where long-scale elasticity of the strongly interacting Goldstone modes (associated with the spontaneously ordered phase) is characterized by a nontrivial low-temperature fixed point leading to universal anomalous elasticity.

The best way to experimentally probe the consequences of spontaneously broken symmetry is to break it directly with a weak external field. Here we predict that, as a consequence of the anomalous elasticity, the increase in the magnetic induction $\delta B(H) \equiv B(H)-B(0)$ over the spontaneous induction $B(0)$ as a function of weak applied field $H$ obeys a universal "non-Hookean" scaling law

$$
\delta B(H) \propto H^{\alpha},
$$

with the universal exponent $\alpha=0.72 \pm 0.04$ (see Fig. 1), a prediction that should be experimentally testable. This nonHookean scaling is in contrast to conventional vortex lattices in which $\delta B(H)$ scales approximately linearly with $H-H_{c 1}$ for $H>H_{c 1} .^{28}$

The rest of this paper is organized as follows. In Sec. II, we review the Ginzburg-Landau theory of magnetic superconductors, considering the idealized case of vanishing crystalline pinning anisotropy, ${ }^{18}$ and use it to derive the effective interaction between vortex lines in such materials. Using this result, we show rigorously, using a thermodynamic argument, that as a consequence of the underlying rotational invariance the tilt modulus at long wavelengths $c_{44}\left(q_{z}=0\right)$ vanishes in the spontaneous vortex state, thereby showing that the low-energy, long-wavelength tilt modes of the spontaneous vortex lattice are controlled by curvature elasticity of the form $\kappa\left(\partial_{z}^{2} \mathbf{u}\right)^{2}$ (rather than by a conventional tension type $\left(\partial_{z} \mathbf{u}\right)^{2}$, with $\mathbf{u}(\mathbf{r})$ the transverse displacement field of the vortex lattice), and derive the curvature modulus $\kappa$. In Sec. III, we examine the consequences of the softness of the tilt modes for the physics of the fluctuations and pinning of the spontaneous vortex lattice. We show that although both are strongly enhanced quantitatively, in clean samples we expect the spontaneous vortex lattice phenomenology to remain unmodified. This is because the thermal mean-squared fluctua- 
tions in the SV solid, in three dimensions, are dominated by contributions from short length scales (at which, the spontaneous lattice elasticity should not differ qualitatively from that of a conventional lattice.) In particular, we expect only quantitative changes in the melting curve of a spontaneous vortex lattice in a clean sample. In contrast, we find that the effects of pinning by random impurities differ qualitatively from that of a conventional vortex lattice. Specifically, we show that for $d<d_{u c}^{p}=9 / 2$, (in contrast to $d_{u c}^{p}=4$ in an ordinary vortex lattice) random pinning disorders the spontaneous vortex lattice on length scales larger than the highly anisotropic Larkin domains given by Eqs. (1.1a) and (1.1b) [see also Eqs. (4.14a) and (4.14b)]. In Sec. IV we begin by ignoring the effects of nonlinear elasticity and tilt disorder and show that on length scales longer than $R_{c}^{z, \perp}$, the powerlaw roughness of the vortex lattice crosses over to the logarithmic growth of mean-squared phonon distortions, as in conventional vortex lattices. Next, we include tilt disorder (which is always present) and find that for $d<d_{u c}^{t}=7 / 2$ it leads to a far stronger power-law roughness of the vortex lattice, thereby dominating over the logarithmic roughness caused by the vortex positional pinning. In Sec. V, we show that as a consequence of such strong power-law distortions, the nonlinear elasticity becomes qualitatively important, as indicated by the breakdown of perturbation theory in the presence of an, in principle, arbitrarily weak pinning. These findings are consistent with similar results for other soft solids in the presence of disorder, most notably from studies of liquid crystals confined in a random matrix. ${ }^{22,29}$ In Sec. VI we use the momentum-shell renormalization-group method to determine the consequences of these effects for the longscale properties of the randomly pinned spontaneous vortex lattice. We show that for scales larger than an anisotropic nonlinear crossover length scale $\xi_{z, \perp}^{N L}$, the elasticity becomes anomalous and scales universally with the wave vector. It is controlled by a nontrivial zero-temperature fixed point that is perturbative in $\epsilon=7 / 2-d$. In Sec. VII we analyze the topological stability of the SV solid and argue that the resulting phase in the presence of disorder is a columnar elastic glass $^{30,21}$ (CEG) that is stable against the proliferation of dislocations. The term "elastic glass" describes a phase that, while translationally disordered, retains topological order. Other examples of elastic glasses have been predicted for smectic $^{29,31,32}$ and columnar (discotic) $)^{21,33}$ liquid crystals. Associated with the loss of translational order is a broadening of the Bragg scattering peaks, so that they now exhibit power-law decay rather than delta-function divergences. The absence of even quasisharp Bragg peaks in these systems is the reason we refer to them as elastic glasses rather than Bragg glasses [of which the ordinary (field-induced) disordered vortex lattice is an example $\left.{ }^{34}\right]$. An elastic glass is distinguished from the liquid phase by being free of unbound dislocation loops, which proliferate in the liquid. The glass transition is identified as an "unbinding" of the dislocation loops. This transition is then, qualitatively, very similar to the melting of the flux lattice in the absence of disorder, which can also be thought of as an unbinding of dislocation loops. The only difference is that in the glass problem, the flux lattice is translationally disordered both above and below the transition. The absence of defects below the transition, how- ever, means that the low-temperature solid phase still has a finite shear modulus, leading to glassy behavior. The experimental consequences of this behavior are discussed in Sec. VIII, and Sec. IX contains a discussion of possible future theoretical directions.

\section{GINZBURG-LANDAU PHENOMENOLOGY AND HARMONIC ELASTIC THEORY OF A SPONTANEOUS VORTEX LATTICE}

Irrespective of the microscopic mechanism that is ultimately responsible for the stability of the spontaneous ferromagnetic superconducting state, general symmetry principles dictate that the long length scale phenomenology is described by an effective Landau-Ginzburg free-energy functional

$$
\begin{aligned}
F= & \int d \mathbf{r}\left\{\frac{1}{2} a(T)|\psi|^{2}+\frac{b}{4}|\psi|^{4}+\frac{\hbar^{2}}{2 m}\left|\left(\nabla-i \frac{2 \pi}{\phi_{0}} \mathbf{a}\right) \psi\right|^{2}\right. \\
& +\frac{\mathbf{b}^{2}}{8 \pi}+\frac{1}{2} \alpha(T)|\mathbf{M}|^{2}+\frac{1}{4} \beta|\mathbf{M}|^{4}+\frac{1}{2} \gamma|\nabla \mathbf{M}|^{2}-\mathbf{b} \cdot \mathbf{M} \\
& \left.-\frac{1}{4 \pi} \mathbf{b} \cdot \mathbf{H}\right\},
\end{aligned}
$$

where $\psi(\mathbf{r})$ is the local superconducting order parameter, $\mathbf{M}(\mathbf{r})$ is the magnetization, $\phi_{0}=h c / 2 e$ is the quantum of flux, $\mathbf{a}$ is the vector potential, $\mathbf{b}=\boldsymbol{\nabla} \times \mathbf{a}$ is the total flux density inside the superconductor, $\mathbf{H}$ is the external magnetic field, and we have (for now) ignored crystalline anisotropy. ${ }^{18}$ The constants $a, b, \alpha, \beta$, and $\gamma$ are experimentally accessible phenomenological parameters, with $a(T)$ and $\widetilde{\alpha}=\alpha(T)-4 \pi$ changing sign at the superconducting and ferromagnetic transition temperatures, respectively. ${ }^{35}$ The magnetic field $\mathbf{h}$ associated with the superconducting currents satisfies Ampère's law $\nabla \times \mathbf{h}=4 \pi \mathbf{j} / c$ ( $c$ is the speed of light in vacuum) and is related to $\mathbf{b}$ by

$$
\mathbf{b}(\mathbf{r})=\mathbf{h}(\mathbf{r})+4 \pi \mathbf{M}(\mathbf{r})+\mathbf{H}
$$

The term $|\boldsymbol{\nabla} \mathbf{M}|^{2}$ in Eq. (2.1) stands for $\Sigma_{i}\left(\boldsymbol{\nabla} \mathbf{M}_{i}\right)^{2}$. From Eq. (2.2) we observe that the flux density $\mathbf{b}$ is determined by magnetization of the local moments $\mathbf{M}$, the external magnetic field $\mathbf{H}$, and the screening magnetic field $\mathbf{h}$ generated by the diamagnetic orbital currents $\mathbf{j}$.

The minimization of $F$ with respect to $\psi$, $\mathbf{a}$, and $\mathbf{M}$ leads to the following three coupled equations:

$$
\begin{gathered}
\frac{\hbar^{2}}{2 m}\left(\boldsymbol{\nabla}-i \frac{2 \pi}{\phi_{0}} \mathbf{a}\right)^{2} \psi=-\frac{1}{2} a \psi-\frac{1}{2} b|\psi|^{2} \psi \\
\frac{1}{4 \pi} \boldsymbol{\nabla} \times(\boldsymbol{\nabla} \times \mathbf{a})= \\
=\frac{i e \hbar}{2 m c}\left[\psi \nabla \psi^{*}-\psi^{*} \boldsymbol{\nabla} \psi\right]-\frac{2 e^{2}}{m c^{2}}|\psi|^{2} \mathbf{a} \\
+\nabla \times \mathbf{M}+\frac{1}{4 \pi} \nabla \times \mathbf{H} \\
\mathbf{b}(\mathbf{r})=\alpha \mathbf{M}+\beta|\mathbf{M}|^{2} \mathbf{M}-\gamma \nabla^{2} \mathbf{M}
\end{gathered}
$$

It has been shown many years ago by Kuper et al. ${ }^{12}$ and by Greenside et al. ${ }^{14}$ that, depending on the actual values of 
model parameters, the above mean-field equations admit a variety of equilibrium phases, with transitions between them controlled by the temperature, which enters model parameters, most notably $\alpha(T)$ and $a(T)$. In addition to the paramagnetic normal phase, $M=\psi=0$ appearing for $\widetilde{\alpha}(T)>0$, $a(T)>0$, a pure superconducting state, $M=0, \psi \neq 0$, a ferromagnetic normal state $(M \neq 0, \psi=0)$ and a stable spiral phase (where superconductivity coexists with spiraling magnetization) can appear. Furthermore, the Ginzburg-Landau theory also predicts a thermodynamically stable spontaneous vortex state for a range of physically realistic parameters that give a large Abrikosov ratio required for a robust mixed state [ $\lambda / \xi \sim \mathcal{O}(10), \xi$ being the superconducting coherence length and $\lambda$ the London penetration depth] and a large exchange $\gamma$ necessary to suppress the competing spiral phase. In this mixed state, vortices are induced by the local magnetic moments that are spontaneously ordered into a uniform ferromagnetic state. This is in contrast to conventional vortex lattices, which are induced by an external magnetic field.

It is not our intention in this article to study the rich phase diagram that follows from Eqs. (2.3a), (2.3b), and (2.3c). For this, we refer the interested reader to previous work, ${ }^{12,14}$ where this has been done in great detail. Instead our focus here is on the spontaneous vortex state. We use the Ginzburg-Landau theory to derive the vortex line interaction and elasticity of the resulting spontaneous vortex solid phase and study it in the presence of thermal fluctuations and random pinning.

\section{A. Interaction potential between vortices in the SV state}

The description of the vortex state can, as usual, be derived from the Ginzburg-Landau theory by reexpressing the fields $\psi$ and a in terms of the vortex positions, thereby reexpressing energy in terms of the vortex conformational degrees of freedom. To this end, taking the curl of Eq. (2.2), using Ampère's law $\nabla \times \mathbf{h}=(4 \pi / c) \mathbf{j}$, and $\mathbf{b}=\nabla \times \mathbf{a}$, we obtain

$$
\frac{1}{4 \pi} \boldsymbol{\nabla} \times(\boldsymbol{\nabla} \times a)=\frac{1}{c} \mathbf{j}(\mathbf{r})+\nabla \times \mathbf{M}(\mathbf{r})
$$

for zero external magnetic field.

Comparing this expression to Eq. (2.3b) gives the standard expression for the superconducting current ${ }^{28}$

$$
\mathbf{j}(\mathbf{r})=\frac{i e \hbar}{2 m}\left[\psi \nabla \psi^{*}-\psi^{*} \boldsymbol{\nabla} \psi\right]-\frac{2 e^{2}}{m c}|\psi|^{2} \mathbf{a},
$$

which, in the London approximation $\psi=\psi_{0} e^{i \theta(\mathbf{r})}$ (with $\psi_{0}$ a constant except inside the small vortex core of size $\xi$, which is valid in the large $\lambda / \xi \gg 1$ limit), reduces to ${ }^{28}$

$$
\mathbf{j}(\mathbf{r})=-\frac{c}{4 \pi \lambda^{2}}\left[\mathbf{a}(\mathbf{r})-\frac{\phi_{0}}{2 \pi} \boldsymbol{\nabla} \theta\right] .
$$

This relates superconducting currents to the vector potential and the phase of the superconducting order parameter, with the London penetration depth given by $\lambda=\left(m c^{2} / 4 \pi e^{2} \psi_{0}^{2}\right)^{1 / 2}$. Taking the curl of this current and using Ampère's law, together with Eq. (2.2) and

$$
\boldsymbol{\nabla} \times \boldsymbol{\nabla} \theta=2 \pi \sum_{\nu} \delta_{2}\left[\mathbf{r}-\mathbf{r}_{\nu}(z)\right] \frac{d \mathbf{R}_{\nu}}{d z}
$$

where the sum runs over vortices, with

$$
\mathbf{R}_{\nu}(z)=\left[\mathbf{r}_{\nu}(z), z\right]
$$

parametrizing the trajectory of the $\nu$ th vortex line as it traverses the superconducting sample (we choose the average magnetization and vortex direction to be along the $\hat{\mathbf{z}}$ axis), we find

$$
\mathbf{b}(\mathbf{r})-\lambda^{2} \nabla^{2} \mathbf{b}(\mathbf{r})=\phi_{0} \sum_{\nu} \delta_{2}\left[\mathbf{r}-\mathbf{r}_{\nu}(z)\right] \frac{d \mathbf{R}_{\nu}}{d z}-4 \pi \lambda^{2} \nabla^{2} \mathbf{M}(\mathbf{r}) .
$$

This London equation differs from its counterpart in ordinary superconductors by the presence of the local magnetization term on its right-hand side. It needs to be solved simultaneously with the constitutive relation [Eq. (2.3c)] relating flux-density $\mathbf{b}$ and magnetization $\mathbf{M}$.

Using Eq. (2.3c) and the $\mathbf{H}=0$ version of Eq. (2.2), we can write the following equation for the screening field $\mathbf{h}(\mathbf{r})$ :

$$
\mathbf{h}(\mathbf{r})=\widetilde{\alpha} \mathbf{M}+\beta|\mathbf{M}|^{2} \mathbf{M}-\gamma \nabla^{2} \mathbf{M}
$$

with $\tilde{\alpha}=\alpha-4 \pi$. For small $\mathbf{h}(\mathbf{r})$ and $\tilde{\alpha}<0$ this equation has the spatially uniform solution $\mathbf{M}(\mathbf{r})=\mathbf{h} \sqrt{|\tilde{\alpha}| / \beta} \equiv \mathbf{M}_{0}$. Linearizing about this solution by writing $\mathbf{M}(\mathbf{r})=\mathbf{M}_{0}+\delta M(\mathbf{r})$ and expanding to linear order in $\delta M(\mathbf{r})$ gives, for $\mathbf{q} \neq \mathbf{0}$,

$$
\mathbf{M}(\mathbf{q})=\delta \mathbf{M}(\mathbf{q})=\chi(\mathbf{q}) \mathbf{h}(\mathbf{q})
$$

where the second equality follows from the fact that $\mathbf{M}_{0}$, being spatially uniform, contributes nothing to $\mathbf{M}(\mathbf{q})$ for $\mathbf{q}$ $\neq \mathbf{0}$, and

$$
\chi(q) \equiv \frac{1}{2|\tilde{\alpha}(T)|+\gamma q^{2}}
$$

Combining this equation with Eq. (2.9) gives, in Fourier space

$$
\left(1+\lambda^{2} q^{2}\right) \mathbf{b}(\mathbf{q})=\phi_{0} \sum_{\nu} \int d z e^{-i \mathbf{q} \cdot \mathbf{R}_{\nu}(z)} \frac{d \mathbf{R}_{\nu}}{d z}+4 \pi \lambda^{2} q^{2} \chi(\mathbf{q}) \mathbf{h}(\mathbf{q})
$$

Now, using Eq. (2.11) together with the $\mathbf{H}=0$ version of $\mathrm{Eq}$. (2.2) gives

$$
\mathbf{b}(\mathbf{q})=[1+4 \pi \chi(\mathbf{q})] \mathbf{h}(\mathbf{q}),
$$

which allows us to solve for the magnetic field $\mathbf{h}(\mathbf{q})$ and flux density $\mathbf{b}(\mathbf{q})$

$$
\mathbf{h}(\mathbf{q})=\phi_{0} \sum_{\nu} \int d z \frac{e^{-i \mathbf{q} \cdot \mathbf{R}_{\nu}(z)}}{1+\lambda^{2} q^{2}+4 \pi \chi(\mathbf{q})} \frac{d \mathbf{R}_{\nu}}{d z}
$$




$$
\mathbf{b}(\mathbf{q})=\phi_{0} \sum_{\nu} \int d z \frac{[1+4 \pi \chi(\mathbf{q})] e^{-i \mathbf{q} \cdot \mathbf{R}_{\nu}(z)}}{1+\lambda^{2} q^{2}+4 \pi \chi(\mathbf{q})} \frac{d \mathbf{R}_{v}}{d z} .
$$

It is easy to verify that, for one flux line, $\int d \mathbf{x} b_{z}(\mathbf{x}, z)=\phi_{0}$, giving the magnetic flux quantization for a single vortex. On the other hand, the magnetic field $\mathbf{h}(\mathbf{r})$, because of the screening currents around the vortex cores, satisfies $\int d \mathbf{x} h_{z}(\mathbf{x}, z) \simeq \phi_{0} /[1+4 \pi \chi(0)] \neq \phi_{0}$ and is therefore not quantized.

To proceed further we use the London approximation for the kinetic-energy density

$$
\begin{aligned}
\frac{\hbar^{2}}{2 m}\left|\left(\nabla-\frac{2 i \pi}{\phi_{0}} \mathbf{a}\right) \psi\right|^{2} & =\frac{2 \pi \lambda^{2}}{c^{2}} j^{2}, \\
& =\frac{\lambda^{2}}{8 \pi}(\nabla \times \mathbf{h})^{2}
\end{aligned}
$$

to reduce the $\mathbf{H}=0$ Ginzburg-Landau free-energy functional (2.1) to the London expression, omitting a constant associated with the condensation energy, obtaining:

$$
\begin{aligned}
F_{L}= & \int d \mathbf{r}\left[\frac{\hbar^{2}}{2 m}\left|\left(\boldsymbol{\nabla}-\frac{2 i \pi}{\phi_{0}} \mathbf{a}\right) \psi\right|^{2}+\frac{\mathbf{b}^{2}}{8 \pi}+\frac{1}{2} \alpha(T)|\mathbf{M}|^{2}\right. \\
& \left.+\frac{1}{4} \beta|\mathbf{M}|^{4}+\frac{1}{2} \gamma|\boldsymbol{\nabla} \mathbf{M}|^{2}-\mathbf{b} \cdot \mathbf{M}\right], \\
= & \int d \mathbf{r}\left[\frac{\lambda^{2}}{2 \pi}(\nabla \times \mathbf{h})^{2}+\frac{\mathbf{b}^{2}}{8 \pi}+\frac{1}{2} \alpha(T)|\mathbf{M}|^{2}+\frac{1}{4} \beta|\mathbf{M}|^{4}\right. \\
& \left.+\frac{1}{2} \gamma|\boldsymbol{\nabla} \mathbf{M}|^{2}-\mathbf{b} \cdot \mathbf{M}\right] .
\end{aligned}
$$

Using the fact that $\mathbf{b}=\mathbf{h}+4 \pi \mathbf{M}$, the London free energy reduces to a sum $F_{L}=F_{s}+F_{m}$, where (we henceforth use the shorthand notation $\left.\int_{\mathbf{q}}=\int\left[d^{3} \mathbf{q} /(2 \pi)^{3}\right]\right)$

$$
\begin{aligned}
F_{s} & =\frac{1}{8 \pi} \int d^{3} \mathbf{r}\left[h^{2}(\mathbf{r})+\lambda^{2}(\nabla \times \mathbf{h})^{2}\right], \\
& =\int_{\mathbf{q}} \frac{1+\lambda^{2} q^{2}}{8 \pi}|\mathbf{h}(\mathbf{q})|^{2}
\end{aligned}
$$

is the free energy associated with superconducting currents, and

$$
\begin{aligned}
F_{m} & =\int d^{3} \mathbf{r}\left(\frac{1}{2} \widetilde{\alpha}|\mathbf{M}|^{2}+\frac{1}{4} \beta|\mathbf{M}|^{4}+\frac{1}{2} \gamma|\nabla \mathbf{M}|^{2}\right), \\
& =\int_{\mathbf{q}} \frac{1}{2} \chi(\mathbf{q})|\mathbf{h}(\mathbf{q})|^{2}
\end{aligned}
$$

is the ferromagnetic part. We thus obtain

$$
F_{L}=\frac{1}{2} \int_{\mathbf{q}}\left[\frac{1+\lambda^{2} q^{2}}{4 \pi}+\chi(\mathbf{q})\right]|\mathbf{h}(\mathbf{q})|^{2}
$$

Now, using the expression (2.15a) for $\mathbf{h}(\mathbf{q})$ we finally obtain the London free energy of an arbitrary conformation of interacting flux lines

$$
F_{L}=\frac{1}{2} \sum_{\mu \nu} \int d z \int d z^{\prime} \frac{d \mathbf{R}_{\mu}}{d z} \cdot V\left[\mathbf{R}_{\mu}(z)-\mathbf{R}_{\nu}\left(z^{\prime}\right)\right] \frac{d \mathbf{R}_{\nu}}{d z^{\prime}}
$$

with the interaction potential

$$
V\left[\mathbf{R}_{\mu}(z)-\mathbf{R}_{\nu}\left(z^{\prime}\right)\right]=\frac{\phi_{0}^{2}}{4 \pi} \int_{\mathbf{q}} \frac{e^{-i \mathbf{q} \cdot\left[\mathbf{R}_{\mu}(z)-\mathbf{R}_{\nu}\left(z^{\prime}\right)\right]}}{1+\lambda^{2} q^{2}+4 \pi \chi(\mathbf{q})} .
$$

In the absence of the ferromagnetic component $[\chi(\mathbf{q})=0]$, $V(\mathbf{q})$ reduces to the usual London interaction between vortices in a conventional isotropic superconductor.

Using our earlier result (2.12) for $\chi(\mathbf{q})$ implies that the interaction potential $V$ is given in Fourier space by

$$
V(\mathbf{q})=\frac{\phi_{0}^{2}}{4 \pi} \frac{2|\tilde{\alpha}|+\gamma q^{2}}{\left(1+\lambda^{2} q^{2}\right)\left(2|\tilde{\alpha}|+\gamma q^{2}\right)+4 \pi} .
$$

Expressions (2.12) and (2.23) are the expressions we shall use below to derive the elastic moduli of the SV lattice.

\section{B. Tilt and curvature moduli, and harmonic elastic theory of a spontaneous vortex lattice}

Having derived the interaction potential between flux lines, we are now in a position to calculate the tilt modulus of the spontaneous vortex lattice and to show that in the absence of an external field, it indeed vanishes identically as dictated by rotational symmetry. ${ }^{18}$ To this end, we shall write the vortex trajectories as

$$
\mathbf{r}_{\nu}(z)=\mathbf{X}_{\nu}+\mathbf{u}\left(\mathbf{X}_{\nu}, z\right)
$$

with $\mathbf{X}_{\nu}$ the equilibrium lattice position of the $\nu$ th flux line and $\mathbf{u}\left(\mathbf{X}_{\nu}, z\right)$ the two component displacement at height $z$ relative to $\mathbf{X}_{\nu}$. Expanding the London free energy $F_{L}$ of the spontaneous vortex system [Eq. (2.21)] up to quadratic order in the displacements $\mathbf{u}\left(\mathbf{X}_{\nu}, z\right)$ leads to $F_{L}=F_{0}+F_{e l}$, where $F_{0}$ is the energy of an undistorted vortex lattice

$$
\begin{aligned}
F_{0} & =\frac{1}{2} \sum_{\mu \nu} \int d z \int d z^{\prime} V\left(\mathbf{X}_{\mu}-\mathbf{X}_{\nu}, z-z^{\prime}\right), \\
& =\frac{1}{2} \Omega n^{2} \sum_{\mathbf{Q}} V(\mathbf{Q}),
\end{aligned}
$$

and $F_{e l}$ is the elastic energy of the lattice, which in Fourier space is given by 


$$
F_{e l}=\frac{1}{2} \int_{\mathbf{q}} u_{\alpha}(\mathbf{q}) \Phi_{\alpha \beta}(\mathbf{q}) u_{\beta}(-\mathbf{q}),
$$

where $\Omega$ is the volume of the system, $n=B / \phi_{0}$ is the average density of flux lines ( $B$ being the magnetic induction), the Q's are the reciprocal lattice vectors, and we have used Einstein's implicit summation convention over the repeated indices $\alpha$ and $\beta$, which run over the two directions ( $x$ and $y$ ) perpendicular to $z$, the mean direction of the flux lines. The elastic matrix $\Phi_{\alpha \beta}(\mathbf{q})$ of the FLL derived from using the expansion (2.24) in the elastic Hamiltonian (2.21) has the usual form ${ }^{20}$

$$
\begin{aligned}
\Phi_{\alpha \beta}(\mathbf{q})= & n^{2} \sum_{\mathbf{Q}}\left\{\left[\left(Q_{\alpha}-q_{\alpha}\right)\left(Q_{\beta}-q_{\beta}\right)+q_{z}^{2} \delta_{\alpha \beta}\right] V(\mathbf{Q}-\mathbf{q})\right. \\
& \left.-Q_{\alpha} Q_{\beta} V(\mathbf{Q})\right\}
\end{aligned}
$$

with $V(\mathbf{q})$ the interaction potential of Eq. (2.23).

As discussed in the Introduction, in a SV state with no external field to explicitly break rotational symmetry, ${ }^{18}$ the underlying rotational invariance guarantees a vanishing of the vortex-line tilt modulus $c_{44}$. We now prove this explicitly for a spontaneous vortex lattice, via a rigorous thermodynamic argument.

Given that the elastic matrix of a hexagonal vortex lattice (which is the lattice type we expect for an isotropic interaction potential) is of the general form ${ }^{20}$ (in the following, and throughout the rest of the paper, $\mathbf{q}_{\perp}$ stands for the projection of $\mathbf{q}$ onto the plane that is orthogonal to $\hat{\mathbf{z}}$, and Greek indices in $q_{\alpha}, q_{\beta}, \ldots$, run over components of $\mathbf{q}_{\perp}$ only)

$$
\Phi_{\alpha \beta}(\mathbf{q})=\left(c_{11}-c_{66}\right) q_{\alpha} q_{\beta}+\delta_{\alpha \beta}\left(c_{44} q_{z}^{2}+c_{66} q_{\perp}^{2}\right),
$$

we see that the tilt modulus $c_{44}(\mathbf{q})$ can be extracted from $\Phi_{\alpha \beta}\left(q_{z} \hat{\mathbf{z}}\right)$, which, according to (2.28), should be simply

$$
\Phi_{\alpha \beta}\left(q_{z} \hat{\mathbf{z}}\right)=c_{44}\left(q_{z} \hat{\mathbf{z}}\right) q_{z}^{2} \delta_{\alpha \beta} .
$$

Setting $\mathbf{q}=q_{z} \hat{\mathbf{z}}$ in our general expression (2.27) for $\Phi_{\alpha \beta}$ gives

$$
\begin{aligned}
\Phi_{\alpha \beta}(\mathbf{q})= & n^{2} \sum_{\mathbf{Q}} Q_{\alpha} Q_{\beta}\left[V\left(\mathbf{Q}-q_{z} \hat{\mathbf{z}}\right)-V(\mathbf{Q})\right] \\
& +q_{z}^{2} \delta_{\alpha \beta} V\left(\mathbf{Q}-q_{z} \hat{\mathbf{z}}\right) .
\end{aligned}
$$

Using the fact that $V(\mathbf{q})$ depends only on the squared magnitude $q^{2}$ of $\mathbf{q}$, we can rewrite this as

$$
\begin{aligned}
\Phi_{\alpha \beta}\left(q_{z} \hat{\mathbf{z}}\right)= & n^{2} \sum_{\mathbf{Q}} Q_{\alpha} Q_{\beta}\left[\tilde{V}\left(Q^{2}+q_{z}^{2}\right)-\tilde{V}\left(Q^{2}\right)\right] \\
& +\tilde{V}\left(Q^{2}+q_{z}^{2}\right) q_{z}^{2} \delta_{\alpha \beta},
\end{aligned}
$$

where $\tilde{V}$ is the interaction potential such that $V(\mathbf{q})=\tilde{V}\left(q^{2}\right)$. Now, using the fact that for a hexagonal lattice

$$
\sum_{\mathbf{Q}} Q_{\alpha} Q_{\beta} f\left(Q^{2}\right)=\frac{1}{2} \sum_{\mathbf{Q}} Q^{2} f\left(Q^{2}\right) \delta_{\alpha \beta},
$$

we can rewrite $(2.31)$ as
$\Phi_{\alpha \beta}\left(q_{z} \hat{\mathbf{z}}\right)=\delta_{\alpha \beta} n^{2} \sum_{\mathbf{Q}} \frac{Q^{2}}{2}\left[\tilde{V}\left(Q^{2}+q_{z}^{2}\right)-\tilde{V}\left(Q^{2}\right)\right]+\tilde{V}\left(Q^{2}+q_{z}^{2}\right) q_{z}^{2}$,

which is exactly of the form required by (2.29), enabling us to identify

$$
c_{44}\left(q_{z}\right)=n^{2} \sum_{\mathbf{Q}} \frac{Q^{2}}{2}\left[\frac{\tilde{V}\left(Q^{2}+q_{z}^{2}\right)-\tilde{V}\left(Q^{2}\right)}{q_{z}^{2}}+\tilde{V}\left(Q^{2}+q_{z}^{2}\right)\right] .
$$

This expression of $c_{44}\left(q_{z}\right)$ has the following limit as $q_{z} \rightarrow 0$ :

$$
c_{44}(0)=n^{2} \sum_{\mathbf{Q}}\left[\frac{Q^{2}}{2} \tilde{V}^{\prime}\left(Q^{2}\right)+\tilde{V}\left(Q^{2}\right)\right] \text {, }
$$

which can be rewritten as ${ }^{20}$

$$
c_{44}(0)=B \frac{\partial}{\partial B}\left[\frac{1}{2} n^{2} \sum_{\mathbf{Q}} V(\mathbf{Q})\right] .
$$

To see this last step more explicitly we first note that keeping the lattice structure hexagonal, increasing the magnetic field B must decrease the lattice constant $a$ (since the flux carried by each vortex line is a fixed quantum of flux $\phi_{0}$ ). Indeed, since for hexagonal lattice $B=\phi_{0} /\left(a^{2} \sqrt{3}\right)$, we see that $Q^{2}$ $\propto 1 / a^{2}(B) \propto B$, for all reciprocal lattice vectors $\mathbf{Q}$. Equivalently,

$$
Q^{2}(B)=Q^{2}\left(B_{0}\right) B / B_{0},
$$

for all reciprocal lattice vectors $\mathbf{Q}$. It then follows that

$$
\begin{aligned}
\left.\frac{\partial \tilde{V}\left(Q^{2}\right)}{\partial B}\right|_{L} & =\left.\tilde{V}^{\prime}\left(Q^{2}\right) \frac{\partial Q^{2}}{\partial B}\right|_{L}, \\
& =\tilde{V}^{\prime}\left(Q^{2}\right) \frac{Q^{2}\left(B_{0}\right)}{B_{0}} \\
& =\tilde{V}^{\prime}\left(Q^{2}\right) \frac{Q^{2}(B)}{B}
\end{aligned}
$$

where $\left.(\partial / \partial B)\right|_{L}$ denotes derivatives with respect to $B$ keeping the lattice structure fixed, and we have used (2.37) in the last two equalities. Now using this result (2.38) and $n$ $=B / \phi_{0}$, we have

$$
\begin{aligned}
B \frac{\partial}{\partial B}\left[\frac{1}{2} n^{2} \sum_{\mathbf{Q}} V(\mathbf{Q})\right] & =\sum_{\mathbf{Q}}\left[\frac{1}{2} n^{2} \tilde{V}^{\prime}\left(Q^{2}\right) Q^{2}+\tilde{V}\left(Q^{2}\right) B n \frac{\partial n}{\partial B}\right] \\
& =c_{44}(0),
\end{aligned}
$$

as claimed earlier.

As can be seen from Eq. (2.25), the quantity in square brackets Eq. (2.39) is nothing but the free-energy density $\left(F_{0} / \Omega\right)$ of a lattice of straight vortex lines, which leads to

$$
c_{44}\left(q_{z}=0\right)=B \frac{\partial}{\partial B}\left(F_{0} / \Omega\right) .
$$

In the presence of an external magnetic field $\mathbf{H}$, thermodynamics dictates that 


$$
\frac{\partial\left(F_{0} / \Omega\right)}{\partial B}=\frac{H}{4 \pi},
$$

and hence we find

$$
c_{44}\left(q_{z}=0\right)=\frac{B H}{4 \pi}
$$

which shows, that for $H=0$ the long-wavelength tilt modulus vanishes identically

$$
c_{44}\left(q_{z}=0\right)=0,
$$

as required by rotational invariance.

Having established the vanishing of the tilt modulus at $q_{z}=0$, let us now find the limiting form of $c_{44}\left(q_{z}\right)$ for small $q_{z}$. Using a Taylor expansion of the right-hand side of Eq. (2.34) near $q_{z}=0$ leads to the following expression:

$$
c_{44}\left(q_{z}\right)=n^{2} q_{z}^{2} \sum_{\mathbf{Q}}\left[\frac{Q^{2}}{4} \tilde{V}^{\prime \prime}\left(Q^{2}\right)+\tilde{V}^{\prime}\left(Q^{2}\right)\right] .
$$

Neglecting the periodicity of the flux lattice and retaining only the $\mathbf{Q}=0$ term, which is usually dominant at high density, in the sum gives

$$
c_{44}\left(q_{z}\right) \simeq \kappa q_{z}^{2},
$$

with

$$
\kappa \simeq \frac{B^{2}}{4 \pi}\left(\frac{\pi \gamma-\tilde{\alpha}^{2} \lambda^{2}}{[2 \pi+|\tilde{\alpha}|]^{2}}\right) .
$$

Hence, the long-wavelength bending energy of the spontaneous vortex lattice is characterized by a curvature (as opposed to tension) elastic energy of the form $\kappa\left(\partial_{z}^{2} \mathbf{u}\right)^{2}$. Note, however, that expressions (2.45a) and (2.45b) for the curvature modulus $\kappa$ are valid only at long wavelengths, i.e., for $q_{z}$ $\rightarrow 0$. At short wavelengths, we can restrict the reciprocal lattice sum in Eq. (2.34) to the $\mathbf{Q}=0$ term (the so-called continuum approximation), upon which the tilt modulus $c_{44}(q)$ takes the following expression:

$$
c_{44}(\mathbf{q}) \simeq \frac{B^{2}}{4 \pi} \frac{1}{1+\lambda^{2} q^{2}+4 \pi \chi(\mathbf{q})},
$$

where we used the fact that $n=B / \phi_{0}$. In the absence of a magnetic component, $\chi(\mathbf{q})=0$ and Eq. (2.46) reduces to the usual result for the tilt modulus in conventional type-II superconductors.

We can now combine this result for the tilt modulus with the standard analysis ${ }^{20}$ usually applied to obtain the compression and shear moduli and thus write down the harmonic elastic energy for the spontaneous vortex lattice. From the general expression of the elastic matrix [Eq. (2.28)], the compression and shear moduli are given by $c_{11}(q)$ $=\Phi_{x x}(q \hat{\mathbf{x}}) / q^{2}$ and $c_{66}(q)=\Phi_{x x}(q \hat{\mathbf{y}}) / q^{2}$, respectively. Hence, using Eq. (2.27) for $\Phi(\mathbf{q})$, we obtain

$$
c_{11}(q)=\frac{n^{2}}{q^{2}} \sum_{\mathbf{Q}}\left[\left(Q_{x}-q\right)^{2} V(\mathbf{Q}-q \hat{\mathbf{x}})-Q_{x}^{2} V(\mathbf{Q})\right],
$$

$$
c_{66}(q)=\frac{n^{2}}{q^{2}} \sum_{\mathbf{Q} \neq 0} Q_{x}^{2}[V(\mathbf{Q}-q \hat{\mathbf{y}})-V(\mathbf{Q})] .
$$

The compression modulus is readily evaluated in the continuum $(\mathbf{Q}=0)$ limit, with the result

$$
c_{11}(q)=\frac{B^{2}}{4 \pi} \frac{1}{1+\lambda^{2} q^{2}+4 \pi \chi(q)},
$$

where we have again used the fact that $n=B / \phi_{0}$. On the other hand, in expression (2.47b) for the shear modulus, expanding $V(\mathbf{Q}-q \hat{\mathbf{y}})$ to second order in $q$ we obtain the following expression:

$$
c_{66}=\frac{n^{2}}{2} \sum_{\mathbf{Q} \neq 0} Q_{x}^{2} \frac{\partial^{2} V(\mathbf{Q})}{\partial Q_{y}^{2}},
$$

a result that should be valid $\left(c_{66}\right.$ being in general weakly dispersive) over the entire Brillouin zone of the SV lattice. These results are identical to the standard expressions for the ordinary (field-induced) vortex lattices in conventional superconductors, apart from the presence of the susceptibility $\chi(q)$, which reduces to a constant at small wave vector and therefore should not qualitatively alter the long-wavelength behavior of the vortex lattice.

Before closing this section, we note that in the expression of the elastic Hamiltonian of the SV lattice [Eq. (2.26)], $\Phi_{\alpha \beta}(\mathbf{q})$ for a triangular lattice can be written in the form

$$
\Phi_{\alpha \beta}(\mathbf{q})=\Phi_{L}(\mathbf{q}) P_{\alpha \beta}^{L}(\mathbf{q})+\Phi_{T}(\mathbf{q}) P_{\alpha \beta}^{T}(\mathbf{q}),
$$

where $P_{\alpha \beta}^{L}(\mathbf{q})=q_{\alpha} q_{\beta} / q_{\perp}^{2}$ and $P_{\alpha \beta}^{T}(\mathbf{q})=\delta_{\alpha \beta}-P_{\alpha \beta}^{L}(\mathbf{q})$ are the longitudinal and transverse projection operators onto the direction of $\hat{\mathbf{q}}_{\perp}=\mathbf{q}_{\perp} / q_{\perp}$ and the perpendicular direction in the $\left(q_{x}, q_{y}\right)$ plane, respectively, and where the longitudinal and transverse parts of the elastic matrix are given by

$$
\begin{gathered}
\Phi_{L}(\mathbf{q})=c_{11}(\mathbf{q}) q_{\perp}^{2}+c_{44}(\mathbf{q}) q_{z}^{2}, \\
\Phi_{T}(\mathbf{q})=c_{66} q_{\perp}^{2}+c_{44}(\mathbf{q}) q_{z}^{2} .
\end{gathered}
$$

Having established the harmonic elastic theory for the spontaneous vortex lattice, we next use it to study the effects of thermal fluctuations and quenched disorder. As discussed in the Introduction, we anticipate these to be significantly enhanced relative to that of a conventional vortex lattice, due to the softness (vanishing of $c_{44}$ ) of the spontaneous vortex solid.

\section{THERMAL FLUCTUATIONS AND MELTING OF A “SOFT" SPONTANEOUS VORTEX LATTICE}

As usual the effect of thermal fluctuations on vortex lattice translational order is encoded in the vortex line meansquared fluctuation $\left\langle u^{2}\right\rangle$ relative to the ideal lattice positions. This quantity determines the level of translational order, namely, the intensity of the Bragg peaks (in, e.g., neutron scattering) through the Debye-Waller factor

$$
W=e^{-Q^{2}\left\langle u^{2}\right\rangle},
$$

with $Q$ the length of the shortest reciprocal-lattice vector. 
Similarly the location of the melting transition $B_{m}(T)$ in the B-T phase diagram can be roughly located by using $\left\langle u^{2}\right\rangle$ through the Lindemann criterion

$$
\left\langle u^{2}\right\rangle=c_{L}^{2} a^{2},
$$

corresponding to thermal root-mean-square fluctuation of vortex lines becoming comparable to the average vortex spacing $a=\sqrt{\phi_{0} / B}$, with the Lindemann constant $c_{L}$ conventionally taken to be on the order of 0.1 .

Within the harmonic approximation (which, as we will find a posteriori, remains valid at low T) $\left\langle u^{2}\right\rangle$ can be easily computed using Eq. (2.26) above. Equipartition (i.e., simple Gaussian integration) gives the standard expression ${ }^{36}$ (we use units such that Boltzmann's constant $k_{B}=1$ )

$$
\left\langle u^{2}\right\rangle \simeq \int_{\mathbf{q}} \frac{T}{c_{66} q_{\perp}^{2}+c_{44}(\mathbf{q}) q_{z}^{2}},
$$

where we have assumed that $c_{11} \gg c_{66}$ (as is usually the case for materials with a high Ginzburg ratio $\lambda / \xi)$. It is clear from Eq. (3.3) that (as in conventional vortex lattices ${ }^{36}$ ) thermal rms vortex fluctuations are dominated by the largest wavevectors near the Brillouin zone boundary, i.e., near $q_{\perp}$ $\simeq \Lambda=\pi / a, q_{z} \simeq 1 / \xi$. At these short length scales we expect the spontaneous vortex lattice to be characterized by a finite tilt modulus, i.e., for $c_{44}\left(q_{z}\right)$ to approach the expression given in Eq. (2.46), which should not be too different from that of a conventional (external magnetic-field-induced) vortex lattice. We therefore conclude that thermal vortex fluctuations $\left\langle u^{2}\right\rangle$ and all other physical observables, such as the melting curve and the strength of Bragg peaks, to be qualitatively (and roughly even quantitatively) unchanged by the longwavelength "softness" $\left[c_{44}\left(q_{z} \rightarrow 0\right) \approx \kappa q_{z}^{2}\right]$ of the spontaneous vortex lattice. We can compare the SV lattice fluctuations with the conventional vortex (CV) lattice fluctuations $\left(\left\langle u^{2}\right\rangle_{C V}\right)$ by treating $\chi(q)$ perturbatively (valid deep in the ferromagnetic phase where $|\widetilde{\alpha}| \gg 1)$

$$
\left\langle u^{2}\right\rangle \simeq\left\langle u^{2}\right\rangle_{C V}+\int_{\mathbf{q}} \frac{T c_{44}^{C V}(\mathbf{q}) q_{z}^{2}}{\left(c_{66} q_{\perp}^{2}+c_{44}^{C V}(\mathbf{q}) q_{z}^{2}\right)^{2}} \frac{4 \pi \chi(\mathbf{q})}{1+\lambda^{2} q^{2}},
$$

where $c_{44}^{C V}$ is the tilt modulus for a convention vortex lattice. This shows that to lowest order in $\chi(\mathbf{q})$ the SV lattice fluctuations are larger, as we would expect because of the additional softness.

\section{RANDOM PINNING WITHIN THE HARMONIC ELASTIC THEORY}

As in a conventional vortex lattice, random pinning couples to the vortex density $\rho(\mathbf{r})$ (positional disorder) and to the local vortex tilt field $\partial_{z} \mathbf{u}$ (tilt disorder, which we discuss in more detail in Sec. IV C), which are proportional to the $z$ and $\perp$ components, respectively, of the local magnetic induction vector field $\mathbf{B}$. The effects of disorder on the spontaneous vortex lattice can therefore be incorporated through the Hamiltonian $H=H_{e l}+H_{d}$, where the elastic energy is given by Eq. (2.26) and the pinning energy is given by ${ }^{37,30}$

$$
H_{d}=\int d \mathbf{r}\left[V_{d}(\mathbf{r}) \rho(\mathbf{r})+\mathbf{h}(\mathbf{r}) \cdot \partial_{z} \mathbf{u}(\mathbf{r})\right],
$$

where $V_{d}(\mathbf{r})$ and $\mathbf{h}(\mathbf{r})$ are the random pinning potentials for positional and tilt disorders, respectively.

As we will see in a moment, unlike the effects of thermal fluctuations studied above, quenched disorder leads to vortex lattice distortions that are dominated by long length scale fluctuations. As a result, we anticipate that the softness associated with the vanishing of the long-wavelength tilt modulus in a SV lattice will have qualitatively important effects. Hence, to study pinning effects, which will be the focus of the rest of the paper, we specialize on the long wavelength form of the elastic Hamiltonian. This is characterized by the elastic matrix $\Phi_{\alpha \beta}(\mathbf{q})=\Phi_{L}(\mathbf{q}) P_{\alpha \beta}^{L}(\mathbf{q})+\Phi_{T}(\mathbf{q}) P_{\alpha \beta}^{T}(\mathbf{q})$ with the following long-wavelength behavior of the longitudinal and transverse components:

$$
\begin{aligned}
& \Phi_{L}(\mathbf{q})=c_{11} q_{\perp}^{2}+\kappa q_{z}^{4}, \\
& \Phi_{T}(\mathbf{q})=c_{66} q_{\perp}^{2}+\kappa q_{z}^{4} .
\end{aligned}
$$

This elastic matrix is unique to the spontaneous vortex lattice, with the curvature modulus $\kappa$ given by Eq. (2.45b).

\section{A. Perturbative treatment of random pinning: Larkin approximation}

The simplest (yet quite revealing) approximation in a study of random pinning of a periodic medium (e.g., a vortex lattice) is the Larkin approximation. This approximation amounts to Taylor expanding the positional pinning energy (which is a nonlinear function of $\mathbf{u}$ ) to linear order in the phonon displacement $u$. This can be done by expressing the vortex density $\rho(\mathbf{r})$ as an explicit function of $\mathbf{u}$

$$
\rho(\mathbf{r})=\operatorname{Re} \sum_{\mathbf{Q}} \tilde{\rho}_{\mathbf{Q}} e^{i \mathbf{Q} \cdot[\mathbf{r}+\mathbf{u}(\mathbf{r})]},
$$

which leads to a positional pinning energy

$$
H_{d \rho}=\int d \mathbf{r} \operatorname{Re} \sum_{\mathbf{Q}} U_{\mathbf{Q}}(\mathbf{r}) e^{i \mathbf{Q} \cdot \mathbf{u}(\mathbf{r})},
$$

where the Q's are the reciprocal lattice vectors of the vortex lattice and

$$
U_{\mathbf{Q}}(\mathbf{r})=\widetilde{\rho}_{\mathbf{Q}} V_{d}(\mathbf{r}) e^{i \mathbf{Q} \cdot \mathbf{r}} .
$$

We expect correlations of $V_{d}(\mathbf{r})$ to be short-ranged. Even if they are not, those of $U_{\mathbf{Q}}(\mathbf{r})$ are, due to the $e^{i \mathbf{Q} \cdot \mathbf{r}}$ factor, as discussed in Ref. 29. This means that one can accurately capture the long length scale physics of the problem by taking them to be zero range and Gaussian, characterized by a second cumulant

$$
\overline{U_{\mathbf{Q}}(\mathbf{r}) U_{\mathbf{Q}^{\prime}}^{*}\left(\mathbf{r}^{\prime}\right)}=\Delta_{\mathbf{Q}} \delta^{d}\left(\mathbf{r}-\mathbf{r}^{\prime}\right) \delta_{\mathbf{Q}, \mathbf{Q}^{\prime}},
$$

where the set of phenomenological parameters $\Delta_{\mathbf{Q}}$ 's depend on the microscopic natures of the impurities, the vortex lattice, and their interactions.

In using the Larkin approximation we expand the periodic nonlinear piece for small $u$, valid only at short length scales 
such that the typical induced vortex displacement $\mathbf{u}_{\mathrm{rms}}$ $=\left\langle\mathbf{u}^{2}\right\rangle$ is small compared to the correlation length of the random potential. With this approximation we can write the pinning energy as

$$
H_{d} \approx \int d \mathbf{r}\left[\mathbf{f}(\mathbf{r}) \cdot \mathbf{u}(\mathbf{r})+\mathbf{h}(\mathbf{r}) \cdot \partial_{z} \mathbf{u}(\mathbf{r})\right]
$$

with $\mathbf{f}(\mathbf{r})$ an effective random force with two-point correlations

$$
\overline{f_{\alpha}(\mathbf{r}) f_{\beta}\left(\mathbf{r}^{\prime}\right)}=\Delta_{f} \delta_{\alpha \beta} \delta\left(\mathbf{r}-\mathbf{r}^{\prime}\right),
$$

where $\Delta_{f}=\Sigma_{\mathbf{Q}} Q^{2} \Delta_{\mathbf{Q}}$. This leads to a total Hamiltonian in this short-scale Larkin regime that is given by

$$
H=H_{e l}+\int d \mathbf{r f}(\mathbf{r}) \cdot \mathbf{u}(\mathbf{r}) .
$$

Above we have dropped the tilt disorder $\mathbf{h}(\mathbf{r})$, since it is clearly subdominant to the uncorrelated random force $\mathbf{f}(\mathbf{r})$ arising from the Larkin approximation to the positional pinning disorder. Standard Gaussian integration then leads to the disorder-averaged phonon correlation function

$$
C^{f}(\mathbf{r})=C_{L}^{f}(\mathbf{r})+C_{T}^{f}(\mathbf{r})=\overline{\left\langle[\mathbf{u}(\mathbf{r})-\mathbf{u}(\mathbf{0})]^{2}\right\rangle},
$$

where the longitudinal and transverse parts $C_{s}^{\Delta}(\mathbf{r})$ (with $s$ $=L, T$ labeling the longitudinal and transverse polarizations, respectively) of $C^{f}(\mathbf{r})$ are given by

$$
\begin{aligned}
C_{s}^{f}(\mathbf{r}) & =\int_{\mathbf{q}} \frac{2 T[1-\cos (\mathbf{q} \cdot \mathbf{r})]}{B_{s} q_{\perp}^{2}+\kappa q_{z}^{4}}+\int_{\mathbf{q}} \frac{2 \Delta_{f}[1-\cos (\mathbf{q} \cdot \mathbf{r})]}{\left[B_{s} q_{\perp}^{2}+\kappa q_{z}^{4}\right]^{2}}, \\
& \simeq 2 \Delta_{f} \int_{\mathbf{q}} \frac{1-\cos (\mathbf{q} \cdot \mathbf{r})}{\left[B_{s} q_{\perp}^{2}+\kappa q_{z}^{4}\right]^{2}},
\end{aligned}
$$

with $B_{L}=c_{11}$ and $B_{T}=c_{66}$ the bulk and shear moduli of the vortex lattice, respectively, and where, in going from the first to the second equation, we have neglected the thermal contribution to $C_{s}^{f}(\mathbf{r})$, which is subdominant to the disorder part at small wave vectors.

Simple dimensional analysis shows that, for $d \leqslant 9 / 2$, the above integrals are dominated by long length scales, justifying our use of long-wavelength elastic theory derived in Sec. II. In $d$ dimensions we find:

$$
\begin{gathered}
C_{s}^{f}\left(\mathbf{r}=\mathbf{R}_{\perp}\right) \approx \frac{\Delta_{f}}{\kappa^{1 / 4} B_{s}^{7 / 4}} R_{\perp}^{(9 / 2)-d}, \\
C_{s}^{f}\left(\mathbf{r}=R_{z} \hat{\mathbf{z}}\right) \approx \frac{\Delta_{f}}{\kappa^{(5-d) / 2} B_{s}^{(d-1) / 2}} R_{z}^{9-2 d},
\end{gathered}
$$

where we have dropped overall numerical factors of order unity, and $z$ and $\perp$ denote directions along and perpendicular to the flux lines, respectively. In the physically relevant case of $d=3$, the above two expressions become

$$
C_{s}^{f}\left(R_{\perp}\right) \approx \frac{\Delta_{f} R_{\perp}^{3 / 2}}{\kappa^{1 / 4} B_{s}^{7 / 4}},
$$

$$
C_{s}^{f}\left(R_{z}\right) \approx \frac{\Delta_{f} R_{z}^{3}}{\kappa B_{s}} .
$$

Equating these correlation functions to the square of the lattice spacing $a^{2}$ leads to highly anisotropic Larkin lengths in the $x y$ and $z$ directions, given, respectively, by

$$
\begin{gathered}
R_{c}^{\perp} \approx\left(\frac{\kappa^{1 / 4} B_{s}^{7 / 4} a^{2}}{\Delta_{f}}\right)^{2 / 3}, \\
R_{c}^{z} \approx\left(\frac{\kappa B_{s} a^{2}}{\Delta_{f}}\right)^{1 / 3} .
\end{gathered}
$$

These lengths characterize the dimensions of ordered Larkin domains beyond which pinning dominates over elastic energy, disrupting the translational order of the spontaneous vortex lattice. The finiteness of these $3 d$ Larkin domains demonstrates the absence of long-range translational order even for arbitrarily weak disorder. By this last criterion, the Larkin lengths also define the range of length scales over which the above random-force perturbation theory, as defined by the Hamiltonian (4.9), is valid. At longer length scales the rms value of $u$ exceeds the lattice spacing, which invalidates the approximation we used in obtaining Eq. (4.7). Also, because the random force dominates over the random tilt disorder, it is the positional pinning (rather than tilt disorder) that determines the size of Larkin domains.

However, as is well known for conventional vortex lattices and other periodic media, ${ }^{38}$ on longer length scales the Larkin approximation highly overestimates the effect of disorder. On scales beyond $R_{c}^{\perp}$ and $R_{c}^{z}$, a more sophisticated approach that takes into account the nonlinearity of the positional disorder is necessary. Next we shall use the replica Gaussian variational method, ${ }^{39,34}$ to treat the long-scale effects of the (seemingly dominant) positional disorder alone. For now, ignoring tilt disorder and nonlinear elasticity, we shall find, that, in spite of the soft elasticity of the tilt modes in a spontaneous vortex lattice, positional disorder leads to a logarithmic growth of the displacement correlation function $C^{f}(\mathbf{r})$ in the physical case of $d=3$ dimensions, as it does in conventional vortex lattices in ordinary superconductors. ${ }^{38,34}$

\section{B. Positional pinning on long length scales}

As discussed above, the perturbative (random-force) treatment of positional disorder breaks down on length scales longer than the Larkin lengths $R_{c}^{\perp}$ and $R_{c}^{z}$ of Eqs. (4.14a) and (4.14b). In this section we shall use a replica variational analysis ${ }^{40,41}$ similar to the one carried out in Refs. 39 and 34 to find the contribution to the long-distance behavior of the two-point correlation function for relative vortex displacement from the seemingly dominant positional disorder alone. Our starting point here is the Hamiltonian

$$
H=\int_{\mathbf{q}} \frac{1}{2}\left[K q_{\perp}^{2}+\kappa q_{z}^{4}\right]|\mathbf{u}(\mathbf{q})|^{2}+\int d \mathbf{r} \operatorname{Re} \sum_{\mathbf{Q}} U_{\mathbf{Q}}(\mathbf{r}) e^{i \mathbf{Q} \cdot \mathbf{u}(\mathbf{r})},
$$

where for simplicity we have used isotropic elasticity with a single in-plane elastic constant $K$ and the positional disorder 
correlations are given by Eq. (4.6). Employing the standard replica "trick" 42 to average over the quenched disorder, ${ }^{38}$ we obtain the following replicated effective Hamiltonian

$$
\begin{aligned}
H_{e f f} \simeq & \sum_{a=1}^{m} \int_{\mathbf{q}} \frac{1}{2}\left[K q_{\perp}^{2}+\kappa q_{z}^{4}\right] \mathbf{u}_{a}(\mathbf{q}) \cdot \mathbf{u}_{a}(-\mathbf{q}) \\
& -\sum_{a, b=1}^{m} \sum_{\mathbf{Q} \neq 0} \int d \mathbf{r} \frac{\Delta_{\mathbf{Q}}}{2 T} \cos \left\{\mathbf{Q} \cdot\left[\mathbf{u}_{a}(\mathbf{r})-\mathbf{u}_{b}(\mathbf{r})\right]\right\},
\end{aligned}
$$

with $a, b$ labeling the $m \rightarrow 0$ replicas, $\mathbf{Q}$ denoting reciprocallattice vectors, and $\Delta_{\mathbf{Q}}$ defined by Eq. (4.6). In the above we have also dropped the coupling of disorder to the long-scale fluctuations in the vortex density $-\rho_{0} \boldsymbol{\nabla} \cdot \mathbf{u}$, that in $3 d$ is subdominant to the short-scale pinning that we have kept.

We now want to study the above nonlinear Hamiltonian via a Gaussian variational approximation ${ }^{39,34}$ with the following harmonic trial Hamiltonian:

$$
H_{v}=\frac{1}{2} \sum_{a b} \int_{\mathbf{q}}\left[G^{-1}(\mathbf{q})\right]_{a b} \mathbf{u}_{a}(\mathbf{q}) \cdot \mathbf{u}_{b}(-\mathbf{q}),
$$

which is parametrized by a variationally determined inverse "propagator"

$$
\left[G^{-1}(\mathbf{q})\right]_{a b}=\left(K q_{\perp}^{2}+\kappa q_{z}^{4}\right) \delta_{a b}-\sigma_{a b},
$$

characterized by an $m \times m$ self-energy matrix $\sigma_{a b}$ of variational parameters, which encodes the average vortex positional correlations.

Minimization of the variational free energy

$$
F_{v}=\left\langle H_{\text {eff }}-H_{v}\right\rangle_{v}-T \ln Z_{v},
$$

where $Z_{v}=\operatorname{Tr}\left[\exp \left(-H_{v} / T\right)\right]$ and $\langle\ldots\rangle_{v}$ denotes averaging with statistical weight $\exp \left(-H_{v} / T\right) / Z_{v}$, leads to saddle-point equations, which are very similar to those derived in Ref. 34. As discussed in more detail there, the long-scale properties of the pinned state are characterized by a replica-symmetrybroken matrix $A_{a b}$ with "hierarchical" structure, that in the $m \rightarrow 0$ limit can be viewed as a single function $\sigma(v)$ of a real variable $v$ in the interval $[0,1]$, that is the key quantity of the replica method. The hierarchical matrix $\sigma(v)$ is a solution of the saddle-point equation (here $Q_{0}$ is the magnitude of the smallest reciprocal lattice vectors)

$$
\sigma(v) \int_{\mathbf{q}} \frac{T Q_{0}^{2}}{\left[\kappa q_{z}^{4}+K q_{\perp}^{2}+[\sigma](v)\right]^{2}}=1,
$$

where we denote by $[\sigma](v)$ the quantity $[\sigma](v)=v \sigma(v)$ $-\int_{0}^{v} d u \sigma(u)$. The solution of the saddle-point Eq. (4.20) proceeds in much the same way as for a conventional, fieldinduced vortex lattice. ${ }^{34}$ We refer the reader to Appendix A where we give more details on this solution and present here only the final results for the disorder-averaged correlation function $C^{p}(\mathbf{r})=\overline{\left.\langle\mathbf{u}(\mathbf{r})-\mathbf{u}(\mathbf{0})]^{2}\right\rangle}$ [we use superscript $p$ in $C^{p}(\mathbf{r})$ to distinguish the long-scale translational correlator in presence of the periodic density pinning alone, i.e., in the absence of tilt disorder and elastic nonlinearities, from the quantity $C^{f}(\mathbf{r})$ of the previous paragraph which corresponds to the Larkin approximation of random pinning forces and which is only valid inside the Larkin domains defined by the length scales $R_{c}^{\perp}$ and $R_{c}^{z}$ of Eqs. (4.14a) and (4.14b)].

For a spontaneous vortex lattice in three spatial dimensions, we find (see Appendix A)

$$
\begin{aligned}
& C^{p}\left(r_{\perp}\right)=\frac{T}{\sqrt{2} u_{0} K \kappa^{1 / 4}} \ln \left(\Lambda r_{\perp}\right), \\
& C^{p}(z)=\frac{T}{\pi \sqrt{2} u_{0} K \kappa^{1 / 4}} \ln (\Lambda z),
\end{aligned}
$$

where (here $d_{\perp}=d-1$ and $c_{d_{\perp}}$ is a numerical constant defined in Appendix A)

$$
u_{0}=\frac{3 \sqrt{2} c_{d_{\perp}} T Q_{0}^{2}}{4\left(7-2 d_{\perp}\right) \kappa^{1 / 4} K^{3 / 2}} .
$$

We thus see that the softness of the tilt modes of the spontaneous vortex lattice does not affect the large distance behavior of the correlation function $C^{p}(\mathbf{r})$, which, like in ordinary (field-induced) disordered vortex lattices, grows only logarithmically at long length scales. This is expected on general grounds, since (weak) disorder-induced logarithmic growth is a consequence of the periodic form of positional pinning in Eq. (4.16), which is guaranteed by the identity symmetry of vortex lines. However, this result is valid only if we ignore the tilt disorder and nonlinear elasticity, which, as we will show below, become important in $d=3$ $<7 / 2$ dimensions. In what follows, we shall consider the effect of random tilt disorder on the spontaneous vortex lattice and show that it leads to power-law growth of the vortex line distortions in $d=3$ dimensions and, therefore, at long scales strongly dominates over the logarithmic growth induced by positional disorder found above.

\section{Random tilt disorder}

In addition to the usual effects of pinning leading to a random coupling to the vortex density [Eq. (4.15)] (corresponding to the pinning of the $z$ component of the magnetic field), it is essential, in studying a spontaneous vortex solid, to include a random tilt disorder, as appearing in $H_{d}$ [Eq. (4.7)]. As schematically illustrated in Fig. 2, physically, such a random term corresponds to local random torques being exerted on the vortex lattice by random, short-range correlated clusters of pinning sites. The interaction with these randomly oriented clusters of point pinners is similar to the interaction of vortex lines with a randomly oriented local transverse magnetic field $\mathbf{h}(\mathbf{r})$ (not to be confused with the screening field around vortices of Sec. II), which couples to the transverse component of the magnetic-induction vector field $\mathbf{B}_{\perp}$, and therefore leads to random coupling

$$
H_{t}=\int d \mathbf{r} \mathbf{h}(\mathbf{r}) \cdot \partial_{z} \mathbf{u}(\mathbf{r})
$$

For simplicity we take $\mathbf{h}(\mathbf{r})$ to be a Gaussian random field with zero mean and correlations 


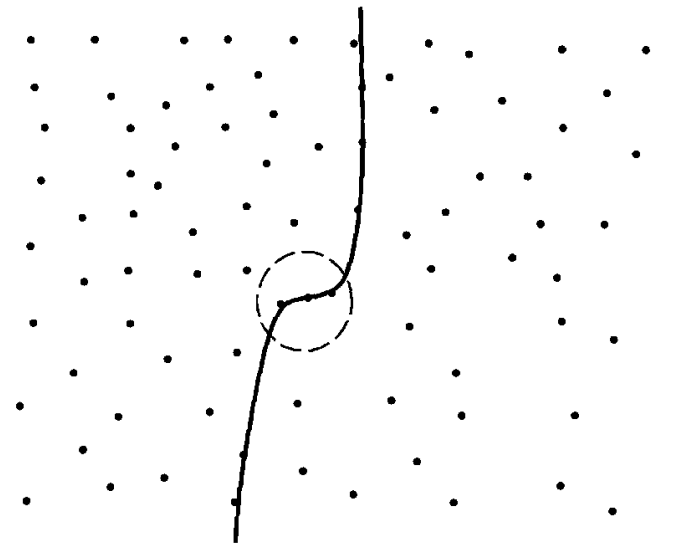

FIG. 2. Schematic representation of the mechanism of random tilt pinning. Randomly oriented anisotropic clusters of point-pins exert random torques on vortex lines.

$$
h_{\alpha}(\mathbf{r}) h_{\beta}\left(\mathbf{r}^{\prime}\right)=\Delta_{t} \delta_{\alpha \beta} \delta\left(\mathbf{r}-\mathbf{r}^{\prime}\right) .
$$

Independent of the microscopic mechanism, it is simple to see that tilt disorder is always generated from the positional disorder upon coarse graining even if it is left out in the original model. Such disorder is generally left out in a treatment of conventional vortex lattice, as in three dimensions it (as well as the aforementioned coupling of disorder to the long-scale fluctuations in the vortex density $\left.-V_{d}(\mathbf{r}) \rho_{0} \boldsymbol{\nabla} \cdot \mathbf{u}\right)$ is subdominant to the positional pinning that we have studied in Sec. IV B. However, as we will show below, because of the softness of the spontaneous vortex lattice (more specifically, the vanishing of its long-wavelength tilt modulus $c_{44}$ ), this tilt disorder leads to power-law distortions of the spontaneous vortex lattice in three dimensions. It is therefore the dominant form of disorder that must be taken into account on long scales.

To see this we treat tilt disorder alone. Because tilt disorder couples only linearly to the phonon $\mathbf{u}$ field (in the absence of positional disorder and nonlinear elasticity) it is straightforward to study its effects on the vortex positional correlations. Using the Hamiltonian $\mathrm{H}=\mathrm{H}_{e l}+\mathrm{H}_{t}$, a standard calculation gives for the longitudinal and transverse contributions of the positional correlation function $C^{t}(\mathbf{r})$ $=\left\langle[\mathbf{u}(\mathbf{r})-\mathbf{u}(\mathbf{0})]^{2}\right\rangle=C_{L}^{t}(\mathbf{r})+C_{T}^{t}(\mathbf{r})$. Here we use the superscript $t$ to distinguish this correlator associated with tilt disorder from the correlation functions $C^{f}$ and $C^{p}$ of Secs. IV A and IV B that were associated with positional disorder. Keeping in mind that the subscript $s$ denotes longitudinal $(L)$ and transverse $(T)$ contributions we find that

$$
\begin{aligned}
& C_{s}^{t}(\mathbf{r})=2 T \int_{\mathbf{q}} \begin{array}{c}
q_{z}^{2}[1-\cos (\mathbf{q} \cdot \mathbf{r})] \\
{\left[\kappa q_{z}^{4}+B_{s} q_{\perp}^{2}\right]}
\end{array}+2 \Delta_{t} \int_{\mathbf{q}} \begin{array}{c}
q_{z}^{2}[1-\cos (\mathbf{q} \cdot \mathbf{r})] \\
{\left[\kappa q_{z}^{4}+B_{s} q_{\perp}^{2}\right]^{2}}
\end{array} \\
& \simeq 2 \Delta_{t} \int_{\mathbf{q}} q_{z}^{2}[1-\cos (\mathbf{q} \cdot \mathbf{r})] \\
& {\left[\kappa q_{z}^{4}+B_{s} q_{\perp}^{2}\right]^{2} }
\end{aligned}
$$

where, in going from the first to the second equation, we have dropped the subdominant thermal part and only kept the $T=0$ random pinning contribution. Asymptotic analysis of the above integrals gives (we again drop overall numerical factors of order unity)

$$
\begin{gathered}
C_{s}^{t}\left(r_{\perp}\right) \approx \begin{array}{c}
\Delta_{t} r_{\perp}^{(7 / 2)-d} \\
\kappa^{3 / 4} B_{s}^{5 / 4},
\end{array} \\
\approx \begin{array}{c}
\Delta_{t} r_{\perp}^{1 / 2} \\
\kappa^{3 / 4} B_{s}^{5 / 4},
\end{array} \\
C_{s}^{t}(z) \approx \begin{array}{c}
\Delta_{t} \\
\kappa^{(5-d) / 2} B_{s}^{(d-1) / 2} z^{7-2 d}
\end{array} \\
\approx \begin{array}{l}
\Delta_{t} z \\
\kappa B_{s},
\end{array}
\end{gathered}
$$

where in Eqs. (4.26b) and (4.26d) we have specialized to the physically relevant case of three dimensions. Defining tiltdisorder spatial coherence lengths $\left(\xi_{t}^{\perp}, \xi_{t}^{z}\right)$ as the lengths for which $C_{s}^{t}\left(\xi_{t}^{\perp}, \xi_{t}^{z}\right)=a^{2}$, we find

$$
\begin{aligned}
\xi_{t}^{\perp} & \simeq \begin{array}{c}
B_{s}^{5 / 2} \kappa^{3 / 2} \\
\Delta_{t}^{2}
\end{array} a^{4} \\
\xi_{t}^{z} & \simeq\left(\begin{array}{c}
\kappa B_{s} \\
\Delta_{t}
\end{array}\right) a^{2} .
\end{aligned}
$$

We stress that (in the absence of positional disorder, elastic nonlinearities, and dislocations) the power-law growth of the correlation function $C_{s}^{t}(\mathbf{r})$ [Eqs. (4.26b), (4.26c), and (4.26d)] extends out to arbitrarily long scales. This is in contrast to the Larkin random-force approximation of Sec. IV A. Hence at long length scales, for $d=3<7 / 2, C^{t}(\mathbf{r})$ quite clearly dominates over the much slower, logarithmically growing distortions $C^{p}(\mathbf{r})$ [Eqs. (4.21a) and (4.21b)] created by translational disorder alone. This is a very important point and is one that cannot be seen just by comparing the coherence lengths $\left(\xi_{t}\right)$ in the presence of tilt disorder alone [given by Eqs. (4.27a) and (4.27b)] with those $\left(\xi_{p}\right)$ in the presence of positional disorder alone [given by Eqs. (4.14a) and (4.14b)]. For equally weak disorder strengths (i.e., small $\Delta_{t}$ and $\Delta_{f}$ ), one can see that $\xi_{t} \gg \xi_{p}$ and Larkin length is determined by the positional disorder, i.e., $\xi_{p}=R_{c}$. However, at length scales beyond this Larkin length (where the Larkin approximation breaks down) the vortex lattice distortions are controlled by the random tilt (rather than positional) disorder. ${ }^{43}$ Therefore, henceforth we shall ignore translational disorder altogether and focus on tilt disorder only. As we will show below, despite this considerable simplification, the asymptotic properties of the spontaneous vortex lattice remain nontrivial and rich because of the interplay of tilt disorder and anharmonic elasticity that we have ignored thus far.

\section{PERTURBATIVE TREATMENT OF ELASTIC NONLINEARITIES AND TILT DISORDER}

The elasticity of a pinned line crystal can be most easily formulated in terms of the (Lagrangian) left Cauchy-Green strain tensor $v_{\alpha \beta},{ }^{44}$ defined by 


\section{$-4 / 5$}

$-0.033$

$\mathrm{x}$

FIG. 3. Renormalization group flow of the ratio of the Lamé coefficients $x=\lambda / \mu$. The fixed point at $x_{2}=-0.033$ controls the long-scale properties (anomalous elasticity and associated phenomenology) of the pinned spontaneous vortex solid. The critical point at $x_{1}=-4 / 5$ controls the mechanical instability of the spontaneous vortex solid.

$$
v_{\alpha \beta}={ }_{2}^{1}\left(\partial_{i} R_{\alpha} \partial_{i} R_{\beta}-\delta_{\alpha \beta}\right) .
$$

For a line-vortex solid characterized by a conformation $\mathbf{R}\left(\mathbf{X}_{\mu}, z\right)$, defined in Eqs. (2.8) and (2.24), the nonlinear Lagrangian strain tensor reduces to

$$
v_{\alpha \beta}={ }_{2}^{1}\left(\partial_{\alpha} u_{\beta}+\partial_{\beta} u_{\alpha}+\partial_{i} u_{\alpha} \partial_{i} u_{\beta}\right),
$$

with $\alpha, \beta$ ranging over the $d_{\perp}$-dimensional subspace $(x, y)$ transverse to the average spontaneous vortex line direction, which we take to be $\hat{\mathbf{z}}$, and $i$ ranging over the full space $(x, y, z)$. In conventional elastic solids, for weak disorder and low temperature the gradients of the phonon field $\mathbf{u}(\mathbf{r})$ are usually small and it suffices to approximate the full nonlinear strain tensor by its harmonic part $v_{\alpha \beta}^{0}={ }_{2}^{1}\left(\partial_{\alpha} u_{\beta}+\partial_{\beta} u_{\alpha}\right)$. However, there exists a different class of "soft" elastic systems ${ }^{22}$ in which nonlinear elasticity plays an essential qualitative role. A unifying feature of solids in this class is their underlying, spontaneously broken rotational invariance, which strictly enforces a particular softness of the corresponding Goldstone-mode Hamiltonian. As a consequence, the usually small nonlinear elastic terms are, in fact, comparable to harmonic ones and therefore must be taken into account. Similar to their effects near continuous phase transitions, but extending throughout an ordered phase, fluctuations and disorder drive elastic nonlinearities to qualitatively modify such soft states. The resulting strongly interacting ordered states at long length scales exhibit rich phenomenology, such as a universally nonlocal elasticity, a strictly nonlinear response to an arbitrarily weak perturbation, and a universal ratio of wave-vector-dependent singular elastic moduli, all controlled by nontrivial infrared stable fixed points, illustrated for the problem at hand in Fig. 3. As we have recently argued in a brief communication, ${ }^{30}$ a spontaneous vortex solid, with its symmetry-enforced vanishing tilt modulus $c_{44}$ is a member of the class of such soft solids. Hence, as we will show below, to understand its pinned state the nonlinear part of the strain tensor $v_{\alpha \beta}$, Eq. (5.2) must be taken into account. In this section we demonstrate the importance of nonlinear elastic terms via a simple perturbation theory in these nonlinearities and tilt disorder.

The full nonlinear elastic Hamiltonian of a spontaneous vortex lattice pinned by the tilt disorder is given by (here the Lamé elastic constant $\lambda$ should not be confused with the London penetration depth)

$$
H=\int d \mathbf{r}\left[{ }_{2}^{1} \kappa\left(\partial_{z}^{2} \mathbf{u}\right)^{2}+{ }_{2}^{\lambda} v_{\alpha \alpha}^{2}+\mu v_{\alpha \beta}^{2}+\mathbf{h}(\mathbf{r}) \cdot \partial_{z} \mathbf{u}(\mathbf{r})\right],
$$

where $\kappa$ is the curvature modulus, and $\mu=c_{66}$ and $\lambda=c_{11}$ $-c_{66}$ are Lamé coefficients, respectively, characterizing the in-plane shear and in-plane bulk moduli of the spontaneous vortex solid. The key feature that distinguishes the spontaneous vortex solid from a conventional field-induced one, and requires us to keep nonlinear terms in $\partial_{z} u_{\alpha}$, is the absence of the $\mu_{z \perp} v_{z \alpha}^{2}$ out-of-plane shear term. This is because of the exact vanishing (enforced by rotational symmetry) of the tiltshear modulus $\mu_{z \perp}=c_{44}$. In contrast, because $\mu$ and $\lambda$ are finite, the corresponding elastic nonlinearities in $\partial_{\alpha} u_{\beta}$ need not be retained, as they are clearly subdominant at long length scales. Consequently, for a spontaneous vortex solid the nonlinear elastic strain that appears in $H$ [Eq. (5.3)] is given by

$$
v_{\alpha \beta} \simeq{ }_{2}^{1}\left(\partial_{\alpha} u_{\beta}+\partial_{\beta} u_{\alpha}+\partial_{z} u_{\alpha} \partial_{z} u_{\beta}\right) .
$$

The Hamiltonian $H$ above can be written as

$$
H=H_{0}+H_{1}+H_{d},
$$

where

$$
\begin{aligned}
H_{0}= & \int d \mathbf{r}\left[{ }_{2}^{1} \kappa\left(\partial_{z}^{2} \mathbf{u}\right)^{2}+{ }_{2}^{1} \mu\left(\partial_{\alpha} u_{\beta}\right)^{2}+{ }_{2}^{1} \mu\left(\partial_{\alpha} u_{\beta}\right)\left(\partial_{\beta} u_{\alpha}\right)\right. \\
& \left.+{ }_{2}^{1} \lambda\left(\partial_{\alpha} u_{\alpha}\right)^{2}\right]
\end{aligned}
$$

is the standard harmonic elastic part, while

$$
\begin{aligned}
H_{1}= & \int d \mathbf{r}\left[\mu \partial_{\alpha} u_{\beta} \partial_{z} u_{\alpha} \partial_{z} u_{\beta}+{ }_{2}^{\lambda}\left(\partial_{\alpha} u_{\alpha}\right)\left(\partial_{z} u_{\beta}\right)^{2}\right. \\
& \left.+\frac{1}{8}(\lambda+2 \mu)\left(\partial_{z} u_{\alpha}\right)^{2}\left(\partial_{z} u_{\beta}\right)^{2}\right]
\end{aligned}
$$

is the nonlinear elastic contribution, and

$$
H_{d}=\int d \mathbf{r} \mathbf{h}(\mathbf{r}) \cdot \partial_{z} \mathbf{u}(\mathbf{r})
$$

is the previously introduced random tilt disorder. The quenched tilt disorder can be most efficiently treated via the standard replica trick, ${ }^{42,46}$

$$
Z^{n}=\lim _{n \rightarrow 0} Z^{n}-1
$$

which allows us to formally average over disorder and therefore work with a more convenient translationally invariant field theory. Introducing $n$ replica fields labeled by $a$ and averaging the resulting "partition function," $Z^{n}$ over the tilt disorder $\mathbf{h}(\mathbf{r})$, we find

$$
Z^{n}=\int\left[d \mathbf{u}_{1}(\mathbf{r})\right] \cdots\left[d \mathbf{u}_{n}(\mathbf{r})\right] e^{-\beta\left(H_{0 n}+H_{1 n}\right)},
$$

where $\beta=1 / T$, and

$$
H_{1 n}=\sum_{a=1}^{n} H_{1}\left[\mathbf{u}_{a}\right]
$$

and we denote by $H_{0 n}$ the following (quadratic) Hamiltonian: 


$$
H_{0 n}=\sum_{a, b} \int_{\mathbf{q}} \frac{1}{2} \Gamma_{\alpha \beta}^{a b}(\mathbf{q}) u_{\alpha}^{a}(\mathbf{q}) u_{\beta}^{b}(-\mathbf{q}),
$$

with the kernel

$$
\Gamma_{\alpha \beta}^{a b}(\mathbf{q})=\Phi_{\alpha \beta}(\mathbf{q}) \delta_{a b}-\frac{\Delta_{t}}{T} q_{z}^{2} \delta_{\alpha \beta},
$$

where $\quad \Phi_{\alpha \beta}(\mathbf{q})=\Phi_{L}(\mathbf{q}) P_{\alpha \beta}^{L}(\mathbf{q})+\Phi_{T}(\mathbf{q}) P_{\alpha \beta}^{T}(\mathbf{q}) \quad$ and $\quad \Phi_{L}(\mathbf{q})$ and $\Phi_{T}(\mathbf{q})$ are given by Eqs. (4.2a) and (4.2b). From Eq. (5.13) the noninteracting elastic propagator $G_{\alpha \beta}(\mathbf{q})$ $=\Omega^{-1}\left\langle u_{\alpha}(\mathbf{q}) u_{\beta}(-\mathbf{q})\right\rangle_{0}(\Omega$ being the system volume) can be easily obtained (see Appendix B) and is given in the limit $n \rightarrow 0$ by

$$
G_{\alpha \beta}^{a b}(\mathbf{q})=G_{L}^{a b}(\mathbf{q}) P_{\alpha \beta}^{L}(\mathbf{q})+G_{T}^{a b}(\mathbf{q}) P_{\alpha \beta}^{T}(\mathbf{q}),
$$

where

$$
\begin{aligned}
& G_{L}^{a b}(\mathbf{q})=T \Gamma_{L}^{-1}(\mathbf{q}) \delta_{a b}+\Delta_{t} q_{z}^{2} \Gamma_{L}^{-2}(\mathbf{q}), \\
& G_{T}^{a b}(\mathbf{q})=T \Gamma_{T}^{-1}(\mathbf{q}) \delta_{a b}+\Delta_{t} q_{z}^{2} \Gamma_{T}^{-2}(\mathbf{q}),
\end{aligned}
$$

with the kernels

$$
\begin{gathered}
\Gamma_{L}(\mathbf{q})=(\lambda+2 \mu) q_{\perp}^{2}+\kappa q_{z}^{4}, \\
\Gamma_{T}(\mathbf{q})=\mu q_{\perp}^{2}+\kappa q_{z}^{4} .
\end{gathered}
$$

To assess the importance of elastic nonlinearities we coarse grain $\overline{Z^{n}}$. To this end we decompose the displacement field $\mathbf{u}(\mathbf{r})$ into low and high wave vector parts

$$
\mathbf{u}(\mathbf{r})=\mathbf{u}^{<}(\mathbf{r})+\mathbf{u}^{>}(\mathbf{r}) .
$$

We then perturbatively (in the nonlinear elastic terms) integrate out the quickly varying, short-scale components $\mathbf{u}^{>}(\mathbf{r})$, and thereby obtain a representation of $\overline{Z^{n}}$

$$
\begin{aligned}
\overline{Z^{n}} & =\int\left[d \mathbf{u}_{a}^{<}(\mathbf{r})\right] e^{-\beta H_{0, n}^{<}} Z_{0}^{>}\left\langle e^{-\beta H_{1 n}\left[u^{<}+u^{>}\right]}\right\rangle_{0>}, \\
& =\int\left[d \mathbf{u}_{a}^{<}(\mathbf{r})\right] e^{-\beta H_{\text {eff }},}
\end{aligned}
$$

in terms of a coarse-grained Hamiltonian $H_{\text {eff }}$

$$
H_{\mathrm{eff}}[\mathbf{u}]=H_{0, n}^{<}[\mathbf{u}]+\left\langle H_{1, n}\right\rangle_{0>}-\frac{1}{2 T}\left\langle H_{1, n}^{2}\right\rangle_{0>}^{c}+\cdots,
$$

where $Z_{0}^{>}=\operatorname{Tr}\left(-\beta H_{0, n}^{>}\right),\langle\ldots\rangle_{0>}$ indicates an average with statistical weight $\exp \left(-\beta H_{0, n}^{>}\right) / Z_{0}^{>},\left\langle H_{1, n}^{2}\right\rangle_{0>}^{c}$ denotes the connected cumulant $\equiv\left\langle H_{1, n}^{2}\right\rangle_{0\rangle}-\left\langle H_{1, n}\right\rangle_{0>}^{2}$, and where short-scale modes extend out to wavelengths of size $L_{z} \times L_{\perp}$.

Restricting our attention here to the leading-order ${ }^{29}$ nonlinear part of $H_{1 n}$,

$$
H_{\mathrm{int}}=\sum_{a=1}^{n} \int d \mathbf{r}\left[\mu \partial_{\alpha} u_{\beta}^{a} \partial_{z} u_{\alpha}^{a} \partial_{z} u_{\beta}^{a}+\frac{\lambda}{2} \partial_{\alpha} u_{\alpha}^{a}\left(\partial_{z} u_{\beta}^{a}\right)^{2}\right],
$$

a standard calculation (with details relegated to Appendix C) shows ${ }^{47}$ that the resulting effective Hamiltonian, $H_{\text {eff }}\left[\mathbf{u}^{<}\right]$, can be put into the same form as the original Hamiltonian, $H_{0 n}[\mathbf{u}]$, but with effective elastic moduli perturbatively corrected by the coarse-graining procedure. For example, as we show in great detail in Appendix C, the lowest-order correction $\delta \mu$ to the bare elastic shear modulus $\mu$ is given by

$$
\begin{aligned}
\delta \mu \approx & -\frac{\mu^{2}}{T d_{\perp}\left(d_{\perp}+2\right)} \int^{>} \frac{d q_{z}}{2 \pi} \int \frac{d^{d-1} \mathbf{q}_{\perp}}{(2 \pi)^{d-1}} q_{z}^{4}\left[2\left[G_{L}^{a b}(\mathbf{q})\right]^{2}\right. \\
& \left.+\left(d_{\perp}^{2}-2\right)\left[G_{T}^{a b}(\mathbf{q})\right]^{2}+2 d_{\perp} G_{L}^{a b}(\mathbf{q}) G_{T}^{a b}(\mathbf{q})\right],
\end{aligned}
$$

where $G_{L}^{a b}$ and $G_{T}^{a b}$ are the noninteracting propagators of Eqs. (5.15a) and (5.15b) and where the $>$ sign on the $q_{z}$ integral indicates that we impose the IR cutoff $1 / L_{z}$, restricting the integration range to the region $\left|q_{z}\right|>1 / L_{z}$. Evaluating the above expression to leading order in the IR cutoff $L_{z}$ (and ignoring the subdominant thermal parts) gives the perturbative relative corrections to Lamé elastic moduli

$$
\frac{\delta \mu}{\mu} \sim \frac{\delta \lambda}{\lambda} \sim \Delta_{t} \mu^{(3-d) / 2} \kappa^{(d-7) / 2} L_{z}^{7-2 d},
$$

that for $d<7 / 2$ diverge with $L_{z}, L_{\perp}$. As we will see in subsequent sections (with details given in Appendix C) similar analysis shows that the perturbative corrections to the curvature modulus $\kappa$ and the tilt-disorder strength $\Delta_{t}$ also diverge with system size like $L_{z}^{7-2 d}$. Thus, at long length scales, perturbation theory breaks down for spatial dimensions smaller than the upper critical dimension

$$
d_{u c}=\frac{7}{2} \text {. }
$$

Note that this is the same as the critical dimension below which positional fluctuations diverge. This is not a coincidence. Rather, it is a consequence of the fact that, in powercounting terms, the leading cubic and quartic anharmonicities that we have kept have the same number of derivatives as the leading harmonic terms. In doing this power counting, note that each $\perp$ derivative counts as two $z$ derivatives, as can be seen from the strongly anisotropic form of the harmonic terms. Given this, the only difference in scaling between the anharmonic and the harmonic terms must come from the extra powers of $\mathbf{u}$ in the anharmonic terms. Hence, the anharmonic terms only become important when $\mathbf{u}$ scales up with increasing distance; i.e., when $\mathbf{u}$ fluctuations grow with length scale, as they do below $d=d_{u c}=\frac{7}{2}$.

We define a nonlinear (crossover) length scale at which perturbation theory breaks down by the value of $L_{z}$ at which the relative corrections to the elastic moduli and disorder variance, $\delta \lambda / \lambda, \delta \mu / \mu, \delta \kappa / \kappa$, and $\delta \Delta_{t} / \Delta_{t}$, become of order 1 . Equation (5.22) leads to the following estimate for this nonlinear crossover length $\xi_{z}^{N L}$ :

$$
\xi_{z}^{N L} \simeq\left(\frac{\kappa^{(7-d) / 2} \mu^{(d-3) / 2}}{\Delta_{t}}\right)^{1 /(7-2 d)},
$$




$$
\simeq\left(\frac{\kappa^{2}}{\Delta_{t}}\right), \text { for } d=3 .
$$

A corresponding perpendicular nonlinear crossover length $\xi_{\perp}^{N L}$ can also be defined by imposing a $1 / L_{\perp}$ infrared cutoff on the $\mathbf{q}_{\perp}$ integrals in perturbation theory and is given by

$$
\xi_{\perp}^{N L} \simeq\left(\frac{\mu}{\kappa}\right)^{1 / 2}\left(\xi_{z}^{N L}\right)^{2} .
$$

Hence, we find from the above perturbative coarse-graining procedure that at sufficiently long length scales [greater than $\left.\min \left(\xi_{\perp}^{N L}, \xi_{z}^{N L}\right)\right]$, the anharmonic elasticity becomes qualitatively important, even for arbitrarily weak pinning, thus invalidating the description of the pinned spontaneous vortex solid by a conventional harmonic elasticity theory. In order to describe the pinned spontaneous vortex solid on scales longer than $\left(\xi_{\perp}^{N L}, \xi_{z}^{N L}\right)$, we need to elevate the above perturbation theory to a renormalization group analysis. We turn to this task in Sec. VI.

\section{NONLINEAR ELASTICITY: RG ANALYSIS}

Having established the importance of the nonlinear elasticity (as indicated by the divergent perturbation theory of Sec. V), we now employ the standard Wilson momentumshell renormalization-group method ${ }^{48}$ to study the pinned spontaneous vortex solid on length scales longer than $\xi_{N L}$. The results we derive here were reported earlier in Ref. 30.

\section{A. Recursion relations and zero-temperature fixed points}

To this end, we use phonon mode decomposition [Eq. (5.17)] with high wave-vector modes $\mathbf{u}^{>}$restricted to an infinitesimal cylindrical shell

$$
\begin{gathered}
\Lambda_{z} e^{-\ell}<\left|q_{z}\right|<\Lambda_{z}, \\
0<\left|q_{\perp}\right|<\infty,
\end{gathered}
$$

where $\ell$ is taken to be an infinitesimal positive number. The procedure is to integrate these short length scale modes $\mathbf{u}^{>}(\mathbf{r})$ out of the replicated partition function $\overline{Z^{n}}$ and to interpret the result in terms of a Hamiltonian of the same form but with effective $\ell$-dependent parameters. To simplify the algebra that follows we introduce anisotropic rescalings of lengths and fields:

$$
\begin{gathered}
\mathbf{r}_{\perp}=e^{\omega \ell} \mathbf{r}_{\perp}^{\prime}, \\
z=e^{\ell} z^{\prime}, \\
\mathbf{u}_{\alpha}^{<}(\mathbf{r})=e^{\phi \ell} \mathbf{u}_{\alpha}^{\prime}\left(\mathbf{r}^{\prime}\right) .
\end{gathered}
$$

This leads to zeroth order (in elastic nonlinearities) recursion relations

$$
\begin{aligned}
& \kappa(\ell)=\kappa e^{[(d-1) \omega-3+2 \phi] \ell}, \\
& \lambda(\ell)=\lambda e^{[(d-3) \omega+1+2 \phi] \ell},
\end{aligned}
$$

$$
\begin{aligned}
\mu(\ell) & =\mu e^{[(d-3) \omega+1+2 \phi] \ell}, \\
\frac{\Delta_{t}}{T}(\ell) & =\frac{\Delta_{t}}{T} e^{[(d-1) \omega-1+2 \phi] \ell} .
\end{aligned}
$$

It is convenient (but not necessary) to take advantage of the rescaling freedom $(6.2 \mathrm{a}),(6.2 \mathrm{~b})$, and $(6.2 \mathrm{c})$, and to choose the arbitrary exponent $\phi$ to be

$$
\phi=2-\omega,
$$

so as to keep fixed ${ }^{49}$ the form of the nonlinear strain tensor $v_{\alpha \beta}=\left(\partial_{\alpha} u_{\beta}+\partial_{\beta} u_{\alpha}+\partial_{z} u_{\alpha} \partial_{z} u_{\beta}\right) / 2$.

Integrating out the high wave-vector modes $\mathbf{u}^{>}$to second order ${ }^{47}$ in elastic nonlinearities leads to the following corrections to effective Hamiltonian parameters (with calculation details provided in Appendix C 2):

$$
\begin{gathered}
\delta D=g F_{D}(x) D d \ell, \\
\delta \mu=g F_{\mu}(x) \mu d \ell, \\
\delta \kappa=g F_{\kappa}(x) \kappa d \ell, \\
\delta\left(\frac{\Delta_{t}}{T}\right)=g F_{\Delta_{t}}(x)\left(\frac{\Delta_{t}}{T}\right) d \ell,
\end{gathered}
$$

where $D=\lambda+\mu$, and where we have defined dimensionless couplings $x$ and $g$ such that:

$$
x=\frac{\lambda}{\mu},
$$

$$
g=\frac{\sqrt{2} C_{d-1}}{64} \Delta_{t} \kappa^{(d-7) / 2} \mu^{(3-d) / 2} \Lambda^{2 d-7},
$$

with the latter $(g)$ controlling the perturbative expansion, and where the scaling functions $F_{i}(x)$ are given by

$$
\begin{gathered}
F_{D}(x)=-\frac{1}{30(x+1)^{2}}\left[135 x^{3}+351 x^{2}+316 x+164\right. \\
\left.+\frac{90 x^{3}+234 x^{2}+144 x-64}{(2+x)^{5 / 4}}\right], \\
F_{\mu}(x)=-\frac{2}{15(x+1)}\left[97+17 x-\frac{152+72 x}{(x+2)^{5 / 4}}\right], \quad(6.7 b) \\
F_{\kappa}(x)=\frac{1}{5(x+1)}\left[1686+366 x-\frac{3896+3156 x+580 x^{2}}{(x+2)^{5 / 4}}\right], \\
F_{\Delta_{t}}(x)=\frac{1}{10(x+1)}\left[543+159 x-\frac{614+230 x}{(x+2)^{1 / 4}}\right] .
\end{gathered}
$$

Combining these with the zeroth order rescalings, [Eqs. $(6.3 a),(6.3 b),(6.3 c),(6.3 d)$, and (6.4)] leads to the differential flow equations 


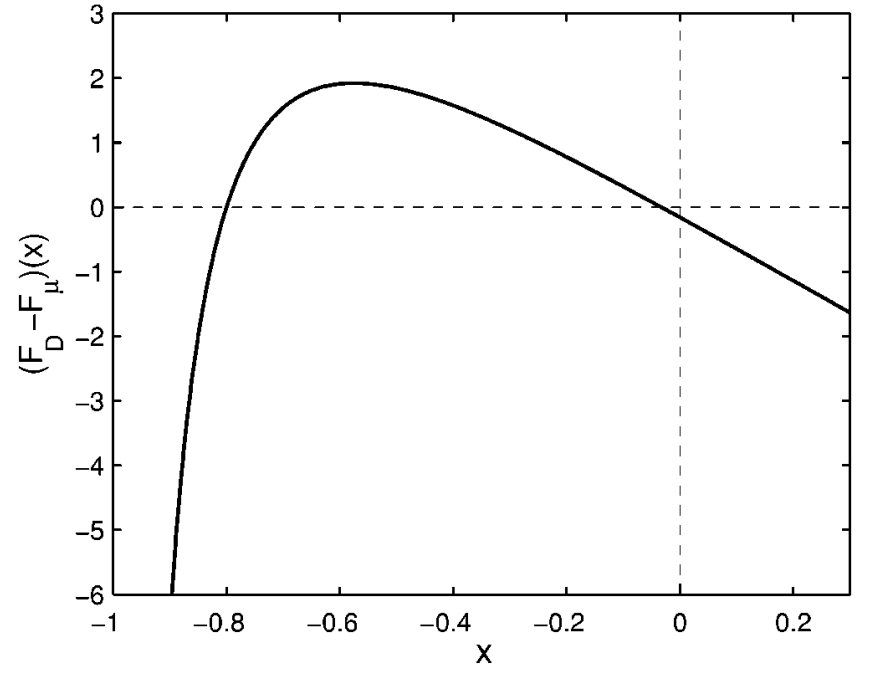

FIG. 4. A plot of the function $\left(F_{D}(x)-F_{\mu}(x)\right)$, with its zeros, $\left(F_{D}(x)-F_{\mu}(x)\right)=0$ at $x_{1}=-4 / 5$ and $x_{2} \simeq-0.03272$ determining the fixed-point solutions for the Lamé coefficients ratio, $x_{*}=\lambda^{*} / \mu^{*}$.

$$
\begin{aligned}
& d D=\left[(d-5) \omega+5+g F_{\lambda}(x)\right] D(\ell), \\
& d \ell \\
& d \mu \\
& d \ell=\left[(d-5) \omega+5+g F_{\mu}(x)\right] \mu(\ell), \\
& d \kappa \\
& d \ell=\left[(d-3) \omega+1+g F_{\kappa}(x)\right] \kappa(\ell), \\
& d \Delta_{t}=\left[(d-3) \omega+3+g F_{\Delta_{t}}(x)\right] \Delta_{t}(\ell) . \\
& d \ell
\end{aligned}
$$

It is easy to verify that the above differential flow equations [Eqs. (6.8a), (6.8b), (6.8c), and (6.8d)] lead to the following two closed flow equations for the two dimensionless couplings $g(\ell)$ and $x(\ell)$ :

$$
\frac{d g}{d \ell}=\left[(7-2 d)+g\left(F_{\Delta_{t}}(x)+\frac{3-d}{2} F_{\mu}(x)+\frac{d-7}{2} F_{\kappa}(x)\right)\right] g .
$$

$$
d \ell=g\left[F_{D}(x)-F_{\mu}(x)\right](x(\ell)+1) .
$$

The fixed points of Eq. (6.9b) [i.e., such that $(d x / d \ell)$ $=0]$ can be easily computed by noting that for finite $g$ they are given by the zeros of the function $\left(F_{\lambda}(x)-F_{\mu}(x)\right)$ plotted in Fig. 4. These are given by

$$
\begin{gathered}
x_{1}=-4 / 5, \\
x_{2} \simeq-0.0327 .
\end{gathered}
$$

As can be seen from Fig. $4,\left(F_{D}(x)-F_{\mu}(x)\right)$ is positive for $x_{1}<x<x_{2}$, and negative otherwise. Therefore $x_{1}=-4 / 5$ is an unstable fixed point, while $x_{2} \simeq-0.0327$ is the stable fixed point of interest to us (see Fig. 3).
At the stable $x_{2} \simeq-0.03272$ fixed point, that we shall denote hereafter by $x_{*}$, the recursion relation $(6.9 \mathrm{a})$ for the dimensionless coupling $g(\ell)$ reduces to

$$
\frac{d g}{d \ell}=[2 \epsilon-12.8359 g] g
$$

with $\epsilon=7 / 2-d$ controlling the validity of the expansion. This implies a stable fixed point [to $\mathcal{O}\left(\epsilon^{2}\right)$ ]

$$
\left(x_{*}, g_{*}\right) \simeq(-0.03272,0.1558 \epsilon),
$$

which controls long-wavelength, weak-disorder, and lowtemperature properties of the pinned spontaneous vortex solid.

The existence of this nontrivial fixed point has dramatic consequences for the long-scale elastic properties of the pinned SV lattice. As discussed above, it leads to the anomalous elasticity (common to soft solids), which we explore below.

The critical point $x_{1}=-4 / 5$ and its instability correspond to the stability limit, in $7 / 2$ dimensions, of the elastic constants, $\lambda$ and $\mu$, beyond which the vortex lattice is unstable against bulk distortions, i.e., $\mathbf{u}(\mathbf{r}) \propto \mathbf{r}_{\perp}$. Stability of the vortex lattice against bulk distortions requires that the bulk modulus, $B=\lambda+\left(2 \mu / d^{*}\right)$ (not to be confused with magnetic flux; $d^{*}=d-1$ is the dimensionality of the vortex lattice) be positive. At the stability limit, i.e., when $B=0$,

$$
x \equiv \stackrel{\lambda}{\mu}=\begin{gathered}
-2 \\
d-1
\end{gathered}
$$

which for $d=7 / 2$ gives $x=-4 / 5$, the value of the unstable fixed point. That the recursion relations reflect this stability limit is a nontrivial check that the $\lambda$ and $\mu$ graphical corrections were correctly evaluated. For $x<-4 / 5$ the flows run away negatively, corresponding to a phase transition from the stable vortex lattice phase to one that, presumably, has different lattice constants. The associated phenomenology would be an interesting subject for future research.

\section{B. Long length-scale correlation functions: Matching}

Many of the basic properties (length-scale-dependent elastic moduli and the associated anomalous elasticity) of the spontaneous vortex glass are encoded in the long-scale limit of the two-point phonon correlation function $G_{\alpha \beta}^{a b}(\mathbf{q})$ $=\left(\Gamma_{\alpha \beta}^{a b}(\mathbf{q})\right)^{-1}$, defined and derived in Appendix B for the harmonic elastic case (valid on length scales shorter than $\xi_{N L}$ ).

We can utilize the renormalization group transformation and the harmonic propagator to compute the correlation function at longer length scales despite it being nonperturbative in the elastic nonlinearities. To this end, recalling the definition of $G_{\alpha \beta}^{a b}(\mathbf{q})$,

$$
G_{\alpha \beta}^{a b}(\mathbf{q})=\begin{gathered}
\left\langle u_{\alpha}^{a}(\mathbf{q}) u_{\beta}^{b}\left(\mathbf{q}^{\prime}\right)\right\rangle \\
(2 \pi)^{d} \delta\left(\mathbf{q}+\mathbf{q}^{\prime}\right)
\end{gathered}
$$

together with the rescalings [Eqs. (6.2a), (6.2b), (6.2c), $(6.3 \mathrm{a}),(6.3 \mathrm{~b}),(6.3 \mathrm{c})$, and $(6.3 \mathrm{~d})]$ it is easy to see that 


$$
\begin{aligned}
G_{\alpha \beta}^{a b} & (\mathbf{q}) \\
& =e^{[(d-1) \omega+1+2 \phi] \ell} \frac{\left\langle u_{\alpha}^{a}\left(\mathbf{q}_{\perp} e^{\omega \ell}, q_{z} e^{\ell}\right) u_{\beta}^{b}\left(\mathbf{q}_{\perp}^{\prime} e^{\omega \ell}, q_{z}^{\prime} e^{\ell}\right)\right\rangle}{(2 \pi)^{d} \delta\left(\mathbf{q}_{\perp} e^{\omega \ell}+\mathbf{q}_{\perp}^{\prime} e^{\omega \ell}\right) \delta\left(q_{z} e^{\ell}+q_{z}^{\prime} e^{\ell}\right)}, \\
& =e^{[(d-3) \omega+5] \ell} G_{\alpha \beta}^{a b}\left(\mathbf{q}_{\perp} e^{\omega \ell}, q_{z} e^{\ell}\right),
\end{aligned}
$$

where in going from the first to the second line we made the convenient choice $\phi=2-\omega$. Finally, integrating out the shortscale phonon modes $u^{>}$gives a scaling relation satisfied by the correlation function

$$
\begin{aligned}
& G_{\alpha \beta}^{a b}\left(\mathbf{q}_{\perp}, q_{z}, \lambda, \mu, \kappa, g\right) \\
& \quad=e^{[(d-3) \omega+5] \ell} G_{\alpha \beta}^{a b}\left(\mathbf{q}_{\perp} e^{\omega \ell}, q_{z} e^{\ell}, \lambda(\ell), \mu(\ell), \kappa(\ell), g(\ell)\right) .
\end{aligned}
$$

This scaling (matching) relation demonstrates the real power of the renormalization-group transformation. ${ }^{50}$ Choosing $e^{\ell}$ large, we can relate the long-wavelength (small $\mathbf{q}$ ) correlation function (which is impossible to compute perturbatively because of the appearance of infrared divergences in the direct perturbation theory) to the correlation function at large q, (e.g., at the Brillouin zone boundary), that can be easily computed using simple perturbation theory.

\section{Long-wavelength behavior of $\kappa$ and $\Delta_{t}$}

To utilize the above scaling relation we first focus on the $q_{\perp}=0$ case and take $\ell^{*}$ such that

$$
q_{z} e^{e^{*}}=\Lambda_{z} \text {. }
$$

We then consider sufficiently long length scales, $\Lambda_{z} / q_{z} \gg 1$, so that $\ell_{*}$ is large enough for the coupling constants $\left[g\left(\ell_{*}\right), x\left(\ell_{*}\right)\right]$ to approach their fixed-point values $\left(g_{*}, x_{*}\right)$. Then using Eq. (6.16) together with the zeroth order approximation for its right-hand side, we find

$$
\begin{aligned}
G_{\alpha \beta}^{a b}\left(q_{\perp}\right. & \left.=0, q_{z}, \lambda, \mu, \kappa, g\right) \\
& =e^{[(d-3) \omega+5] \ell^{*}} G_{\alpha \beta}^{a b}\left(0, \Lambda_{z}, \lambda\left(\ell^{*}\right), \mu\left(\ell^{*}\right), \kappa\left(\ell^{*}\right), g_{*}\right) \\
& =e^{[(d-3) \omega+5] \ell^{*}}\left[\frac{T}{\kappa\left(\ell^{*}\right) \Lambda_{z}^{4}} \delta_{a b}+\frac{\Delta_{t}\left(\ell^{*}\right) \Lambda_{z}^{2}}{\left[\kappa\left(\ell^{*}\right) \Lambda_{z}^{4}\right]^{2}}\right] \delta_{\alpha \beta} .
\end{aligned}
$$

Using Eq. (6.17) to eliminate $\ell_{*}$ in favor of $q_{z}$, the first term in square brackets gives

$$
\begin{aligned}
& e^{[(d-3) \omega+5] e^{*}} \frac{T}{\kappa\left(\ell^{*}\right) \Lambda_{z}^{4}} \delta_{a b} \delta_{\alpha \beta} \\
& =\frac{T e^{[(d-3) \omega+5] e^{*}}}{\kappa q_{z}^{4}\left(\Lambda_{z} / q_{z}\right)^{4} e^{\left[(d-3) \omega+1+g_{*} F_{\kappa}\left(x_{*}\right)\right] e^{*}}} \delta_{a b} \delta_{\alpha \beta}, \\
& =\frac{T\left(\Lambda_{z} / q_{z}\right)^{((d-3) \omega+5)}}{\kappa q_{z}^{4}\left(\Lambda_{z} / q_{z}\right)^{4+\left[(d-3) \omega+1+g_{*} F_{\kappa}\left(x_{*}\right)\right]}} \delta_{a b} \delta_{\alpha \beta}, \\
& =\frac{T}{\kappa q_{z}^{4}\left(\Lambda_{z} / q_{z}\right)^{g_{*} F_{\kappa}\left(x_{*}\right)}} \delta_{a b} \delta_{\alpha \beta},
\end{aligned}
$$

$$
=\frac{T}{\kappa\left(q_{z}\right) q_{z}^{4}} \delta_{a b} \delta_{\alpha \beta} .
$$

We note that as required, the arbitrary rescaling exponent $\omega$ dropped out from the physical correlation function. In the above expression, we can identify $\kappa\left(q_{z}\right)$ as the effective wave-vector-dependent curvature modulus:

$$
\kappa\left(q_{z}\right)=\kappa\left(\frac{q_{z}}{\Lambda_{z}}\right)^{-\eta_{\kappa}},
$$

with the exponent

$$
\eta_{\kappa}=g_{*} F_{\kappa}\left(x_{*}\right),
$$

clearly a universal number determined by the fixed point. A similar reasoning for the second term in (6.18) leads to the wave-vector-dependent disorder variance

$$
\Delta_{t}\left(q_{z}\right)=\Delta_{t}\left(\frac{q_{z}}{\Lambda_{z}}\right)^{-\eta_{\Delta_{t}}}
$$

which corresponds to effective long-range correlated tilt disorder, with a universal exponent given by

$$
\eta_{\Delta_{t}}=g_{*} F_{\Delta_{t}}\left(x_{*}\right) .
$$

From Eqs. (6.21) and (6.23) and the definitions of the functions $F_{\kappa}(x)$ and $F_{\Delta_{t}}(x)$ [Eqs. (6.7c) and (6.7d)], we obtain the following numerical values of the exponents $\eta_{\kappa}$ and $\eta_{\Delta_{t}}$ :

$$
\begin{aligned}
\eta_{\kappa} & =1.478 \epsilon \\
& \simeq 0.739 \text { in } d=3 \text { dimensions }, \\
\eta_{\Delta_{t}} & =0.414 \epsilon \\
& \simeq 0.207 \text { in } d=3 \text { dimensions. }
\end{aligned}
$$

\section{Long-wavelength behavior of $\lambda$ and $\mu$}

Similarly, in order to determine the long-wavelength behavior of the effective Lamé moduli $\lambda$ and $\mu$, we again utilize the scaling relation [Eq. (6.16)]. Here, quite clearly, we need to keep $q_{\perp}$ finite. However, for convenience, we choose $q_{\perp} \xi_{\perp}^{N L} \ll\left(q_{z} \xi_{z}^{N L}\right)^{\zeta}$, so that (as in the previous subsection) it is $q_{z}$ that controls the length-scale dependence of the elastic moduli, and therefore the rescaling parameter $e^{\ell_{*}}$ is still determined by Eq. (6.17),

$$
e^{\ell^{*}}=\left(\frac{\Lambda_{z}}{q_{z}}\right) .
$$

The longitudinal part of $G_{\alpha \beta}^{a b}(\mathbf{q})$ then satisfies

$$
\begin{aligned}
G_{L}^{a b}\left(\mathbf{q}_{\perp}, q_{z}\right) & =e^{[(d-3) \omega+5] \ell^{*}} G_{L}^{a b}\left(q_{\perp} e^{\omega \ell_{*}}, \Lambda_{z}\right) \\
& =\frac{T e^{((d-3) \omega+5) \ell^{*}}}{\left[\lambda\left(\ell^{*}\right)+2 \mu\left(\ell^{*}\right)\right] q_{\perp}^{2} e^{2 \ell_{*}}+\kappa\left(\ell^{*}\right) \Lambda_{z}^{4}} .
\end{aligned}
$$


Utilizing the elastic constant flow equations [Eq. (6.8)], the denominator of the expression on the right-hand side of Eq. (6.26) can be rewritten in the form

$$
\begin{gathered}
e^{[(d-5) \omega+5] e^{*}}\left[\lambda e^{g_{*} F_{\lambda}\left(x_{*}\right) \ell^{*}}+2 \mu e^{g_{*} F_{\mu}\left(x_{*}\right) \ell^{*}}\right] q_{\perp}^{2} e^{2 \ell_{*}} \\
+\kappa q_{z}^{4}\left(\frac{\Lambda_{z}}{q_{z}}\right)^{4} e^{\left[(d-3) \omega+1+g_{*} F_{\kappa}\left(x_{*}\right)\right] e^{*}} .
\end{gathered}
$$

Using Eq. (6.25) we obtain

$$
G_{L}^{a b}\left(\mathbf{q}_{\perp}, q_{z}\right)=\frac{T}{\left[\lambda e^{g_{*} F_{\lambda}\left(x_{*}\right) \ell^{*}}+2 \mu e^{g_{*} F_{\mu}\left(x_{*}\right) \ell^{*}}\right] q_{\perp}^{2}+\kappa q_{z}^{4} e^{g_{*} F_{\kappa}\left(x_{*}\right) e^{*}},}
$$

which shows that at long length scales the longitudinal part of the correlation function $G_{L}^{a b}\left(\mathbf{q}_{\perp}, q_{z}\right)$ takes on its harmonic elasticity form:

$$
G_{L}^{a b}\left(\mathbf{q}_{\perp}, q_{z} ; \lambda, \mu, \kappa, g\right)=\frac{T}{\left[\lambda\left(q_{z}\right)+2 \mu\left(q_{z}\right)\right] q_{\perp}^{2}+\kappa\left(q_{z}\right) q_{z}^{4}},
$$

but with crucial wave-vector-dependent elastic moduli, $\kappa\left(q_{z}\right)$ [Eq. (6.20)] and

$$
\begin{aligned}
& \lambda\left(q_{z}\right)=\lambda\left(\frac{q_{z}}{\Lambda_{z}}\right)^{\eta_{\lambda}}, \\
& \mu\left(q_{z}\right)=\mu\left(\frac{q_{z}}{\Lambda_{z}}\right)^{\eta_{\mu}},
\end{aligned}
$$

where we have defined the anomalous exponents

$$
\eta_{\mu}=\eta_{\lambda}=-g_{*} F_{\mu}\left(x_{*}\right),
$$

which take the universal values:

$$
\begin{aligned}
\eta_{\mu} & =\eta_{\lambda} \simeq 0.6919 \epsilon, \\
& =0.346 \text { for } d=3,
\end{aligned}
$$

controlled by the stable fixed point. The above results show that both Lamé coefficients $\mu$ and $\lambda$ vanish at long wavelengths with the same exponent $\eta_{\mu}$. However, their ratio $x$ $=\lambda / \mu$ flows to a stable fixed point $x_{*}=-0.03272$, implying a universal negative Poisson ratio

$$
\begin{aligned}
\sigma_{P} & =\frac{\lambda}{\lambda+2 \mu}=\frac{x_{*}}{x_{*}+2}, \\
& =-0.0166+O(\epsilon),
\end{aligned}
$$

characterizing the pinned spontaneous vortex solid.

The above calculations can clearly be extended to other directions of $\mathbf{q}$ by choosing a more general $\ell^{*}$ that is a function of both $q_{z}$ and $\mathbf{q}_{\perp}$. To this end we now use a more general choice,

$$
\kappa\left(\ell^{*}\right)\left(q_{z} e^{\ell^{*}}\right)^{4}+\mu\left(\ell^{*}\right)\left(q_{\perp} e^{\omega \ell^{*}}\right)^{2}=\kappa\left(\ell^{*}\right) \Lambda_{z}^{4},
$$

determined by the scaling of the denominator of a transverse part of the phonon correlation function, that for $q_{\perp}=0$ re- duces to the earlier condition [Eq. (6.17)]. On the other hand, for $q_{z}=0$ we obtain the condition [compare to Eq. (6.17)]

$$
q_{\perp} e^{\omega \zeta \ell^{*}}=\Lambda_{z}^{2} \sqrt{\kappa / \mu},
$$

with the universal anisotropy exponent

$$
\zeta=2-\frac{\eta_{\mu}+\eta_{\kappa}}{2} .
$$

Arguments analogous to the ones developed in the previous paragraphs show that the above condition [Eq. (6.34)] leads to the following long-scale behavior of model parameters:

$$
\begin{gathered}
\lambda\left(q_{\perp}\right)=\lambda\left(\frac{q_{\perp}}{\Lambda_{z}^{2}} \sqrt{\frac{\mu}{\kappa}}\right)^{\eta_{\mu} / \zeta}, \\
\mu\left(q_{\perp}\right)=\mu\left(\frac{q_{\perp}}{\Lambda_{z}^{2}} \sqrt{\frac{\mu}{\kappa}}\right)^{\eta_{\mu} / \zeta}, \\
\kappa\left(q_{\perp}\right)=\kappa\left(\frac{q_{\perp}}{\Lambda_{z}^{2}} \sqrt{\frac{\mu}{\kappa}}\right)^{-\eta_{\kappa} / \zeta}, \\
\Delta_{t}\left(q_{\perp}\right)=\Delta_{t}\left(\frac{q_{\perp}}{\Lambda_{z}^{2}} \sqrt{\frac{\mu}{\kappa}}\right)^{-\eta_{\Delta_{t}} / \zeta} .
\end{gathered}
$$

Combining Eqs. (6.20)-(6.22), (6.29a), (6.29b), (6.36a), (6.36b), (6.36c), and (6.36d), we finally see that the general scaling behavior of the model parameters can be written in the form

$$
\begin{gathered}
\lambda(\mathbf{q})=x_{*} \mu\left(q_{z} \xi_{z}^{N L}\right)^{\eta_{\mu}} f_{\mu}\left(q_{\perp} \xi_{\perp}^{N L} /\left(q_{z} \xi_{z}^{N L}\right)^{\zeta}\right), \\
\mu(\mathbf{q})=\mu\left(q_{z} \xi_{z}^{N L}\right)^{\eta_{\mu}} f_{\mu}\left(q_{\perp} \xi_{\perp}^{N L} /\left(q_{z} \xi_{z}^{N L}\right)^{\zeta}\right), \\
\kappa(\mathbf{q})=\kappa\left(q_{z} \xi_{z}^{N L}\right)^{-\eta_{\kappa}} f_{\kappa}\left(q_{\perp} \xi_{\perp}^{N L} /\left(q_{z} \xi_{z}^{N L}\right)^{\zeta}\right), \\
\Delta_{t}(\mathbf{q})=\Delta_{t}\left(q_{z} \xi_{z}^{N L}\right)^{-\eta_{\Delta_{t}}} f_{\Delta_{t}}\left(q_{\perp} \xi_{\perp}^{N L} /\left(q_{z} \xi_{z}^{N L}\right)^{\zeta}\right),
\end{gathered}
$$

where we have taken $\Lambda_{z}=1 / \xi_{z}^{N L}$ (which is a reasonable choice since $\xi_{z}^{N L}$ is the shortest length scale one can go to before perturbation theory can no longer be applied) and used the fact that $\xi_{\perp}^{N L}=\left(\xi_{z}^{N L}\right)^{2}(\mu / \kappa)^{1 / 2}$. The crossover functions $f_{\mu}(x), f_{\kappa}(x)$, and $f_{\Delta_{t}}(x)$ have the following asymptotic behavior:

$$
f_{\mu, \kappa, \Delta_{t}}(x)= \begin{cases}\text { const }, & \text { for } x \ll 1, \\ x^{\eta_{\mu} / \zeta}, x^{-\eta_{\kappa} / \zeta}, x^{-\eta_{\Delta} / \zeta}, & \text { for } x \gg 1 .\end{cases}
$$

\section{Exact relation between exponents}

Since the recursion relation for $g$, Eq. (6.9a) involves functions $F_{\mu, \kappa, \Delta}$ that, respectively, determined the exponents $\eta_{\mu}, \kappa, \Delta_{t}$, it quite clearly leads to an exact relation between these critical exponents. To see this, we note that at the fixed point $\left(x_{*}, g_{*}\right),\left.(d g / d \ell)\right|_{g_{*}}=0$. Combining this with the definitions of the critical exponents [Eqs. (6.30), (6.24a), and $(6.24 c)]$ we obtain an exact exponent relation 


$$
(7-2 d)+\eta_{\Delta_{t}}={ }_{2}^{3-d} \eta_{\mu}+{ }_{2}^{7-d} \eta_{\kappa} .
$$

From a more fundamental perspective, this exact scaling relation (Ward identity) is also necessitated by the underlying rotational invariance (that is spontaneously broken by the vortex lattice). To see this, we recall that rotational invariance requires that the form of the nonlinear strain tensor $v_{\alpha \beta}$ [Eq. (5.2)] be preserved by the perturbation theory in these nonlinearities. Performing the anisotropic rescaling in terms of the physical scaling exponents and imposing this condition (contained in $\phi=2-\omega$ ), we obtain the exact scaling relation, Eq. (6.38).

To order $\epsilon$, Eq. (6.38) is given by

$$
2 \epsilon+\eta_{\Delta_{t}}+{ }_{4}^{1} \eta_{\mu}={ }_{4}^{7} \eta_{\kappa}
$$

and is clearly satisfied by the exponents of Eqs. (6.24b), (6.24d), and (6.31b). In $d=3$ it reduces to

$$
\eta_{\Delta_{t}}=2 \eta_{\kappa}-1,
$$

a relation that, in principle, should be experimentally testable.

\section{TOPOLOGICAL STABILITY OF THE RANDOMLY PINNED SPONTANEOUS VORTEX LATTICE}

We now turn our attention to the important question of whether the phase of the SV lattice, predicted in Sec. VI and that is controlled by the low-temperature fixed point (6.12), is stable against the proliferation of dislocations. It has been shown that long-range translational order in the SV phase is destroyed by arbitrarily weak quenched disorder. This does not necessarily mean, however, that the topology of the phase is destroyed. It is the topology, or "connectivity" of the phase, that is responsible for the phase's elasticity. If the topology is destroyed, then the elasticity is lost. Thus, if the SV lattice remains topologically ordered, it will display SV solidlike elasticity. It will, for example, still have a nonzero shear modulus and shear distortions in the $x y$ plane will still cost energy. We refer to a phase lacking long-range order, but that still has topological order, as an elastic glass..$^{34,51,52}$ Of course, the topological order is eventually lost if the temperature or disorder strength becomes sufficiently large. For sufficiently large temperature $T$ or disorder strength $\Delta$, the topological order of the putative elastic glass will be destroyed via a thermodynamically sharp transition into a translationally and topologically disordered phase.

There are already examples of phase transitions between two phases that both lack long-range order, the most famous being the Kosterlitz-Thouless transition in the $2 d \mathrm{XY}$ model ${ }^{53}$ In that problem, the transition is associated not with the disappearance of long-range order, but rather with the unbinding of neutral pairs of topological defects with increasing temperature. The divergences associated with tilt disorder that destroy the long-range translational order in the columnar elastic glass are, of course, much stronger (power law) than in the $2 d \mathrm{XY}$ model where they are logarithmic.

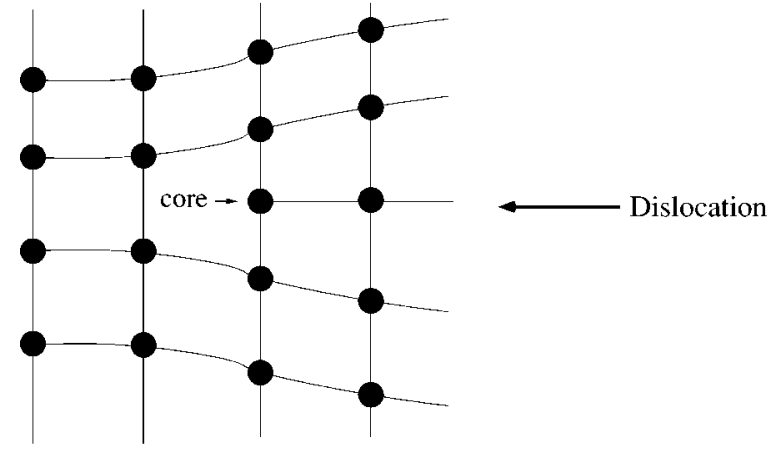

FIG. 5. A square spontaneous vortex with an extra row of flux lines introduced from the right, along the $x$ axis. The core of the dislocation comes out of the page.

There are, however, examples of phases in much more strongly disordered systems in which topological defects, nonetheless, remain bound. ${ }^{54}$ The possibility of such a transition from a translationally disordered smectic-A liquid crystal has also been considered by Radzihovsky and Toner. $^{29}$

In general, the destruction of topological order in a phase occurs via the proliferation of defects. In the $2 d \mathrm{XY}$ model these defects are spin vortices. In the SV lattice the main mechanism for the destruction of topological order is through the proliferation of dislocations. An edge dislocation, which is an insertion of an extra row of flux lines is shown, for a square lattice, in Fig. 5. The Burgers vector (the extra lattice vector) associated with this dislocation is b $=a \hat{y}$. In the remainder of this section, we restrict ourselves to spatial dimensions $d=3$.

This edge dislocation will reduce the elasticity against shears in the $y z$ plane. One could also have an edge dislocation corresponding to multiple extra inserted rows, and in this case the Burgers vector will be a multiple of the lattice vector. If the rows of vortex lines are inserted in the $y z$ plane, then the Burgers vector will be an integer number of lattice vectors in the $\hat{\mathbf{x}}$ direction. One could also have both rows inserted in the $y z$ and the $x z$ planes, and the corresponding Burgers vector would be a sum of lattice vectors. Of course, one can also consider dislocations in a hexagonal lattice, the only difference being that the lattice vectors do not simply point along $\hat{\mathbf{x}}$ and $\hat{\mathbf{y}}$.

When the displacement field $\mathbf{u}(\mathbf{r})$ is integrated around a closed curve enclosing the core of the edge dislocation shown in Fig. 5, the result is not zero as it would be without a dislocation, but rather $\mathbf{b}=\mathbf{a}_{2}=a \hat{\mathbf{y}}$, i.e.,

$$
\oint d \mathbf{u}=\mathbf{a}_{2} \text {. }
$$

In general, a dislocation is a line or curve with the property that an integral of $\mathbf{u}$ around any curve enclosing the dislocation core is some Burgers vector $\mathbf{b}=M \mathbf{a}_{1}+N \mathbf{a}_{2}$, i.e.,

$$
\oint d \mathbf{u}=\mathbf{b}=M \mathbf{a}_{1}+N \mathbf{a}_{2}
$$

where $M$ and $N$ are integers. In the cases of square and hexagonal lattices, in which the lattice vectors are of equal length, Eq. (7.2) can be rewritten as 


$$
\begin{aligned}
& \oint d u_{1}=M a, \\
& \oint d u_{2}=N a,
\end{aligned}
$$

where $u_{i}$ is the $i$ th component of $\mathbf{u}$, defined in the coordinate system with (not necessarily orthogonal) unit vectors $\hat{\mathbf{a}}_{1}$ $=\mathbf{a}_{1} / a, \hat{\mathbf{a}}_{2}=\mathbf{a}_{2} / a$, i.e., $\mathbf{u}=u_{1} \hat{\mathbf{a}}_{1}+u_{2} \hat{\mathbf{a}}_{2}$. For a square lattice $i$ is either $x$ or $y$.

Equations (7.3) apply for all types of dislocations. The edge dislocations, discussed above, have cores that run parallel to the vortex lines but in general dislocation lines can run in any direction. An extreme case is a screw dislocation, which runs perpendicular to the vortices.

\section{A. The defect elastic Hamiltonian}

Equations (7.3a) and (7.3b) imply that in the presence of a dislocation the $\mathbf{u}$ field is no longer single valued. Given that a single-valued $\mathbf{u}$ field is obviously a prerequisite of a meaningful elastic theory, it is necessary to introduce a "cut" in the following fashion. In order to remain single valued as the core of the dislocation is circulated, there must be points at which the value of $\mathbf{u}$ jumps discontinuously by an amount equal to the Burgers vector $\mathbf{b}$. The cut is the locus of points at which this jump occurs, and the value of $\mathbf{u}$ on either side of the cut differs by $\mathbf{b}$. In the absence of dislocations, the elasticity of the system can be described using a field

$$
\mathbf{v}_{i} \equiv \nabla u_{i},
$$

which is continuous and smooth almost everywhere. However, the presence of a cut means that $\mathbf{v}_{i}$ will be singular at the cut. The cut, however, does not represent anything physical and, therefore, nor does a singular $\mathbf{v}_{i}$. There is nothing singularly elastic happening in the real system as the (arbitrarily chosen) cut is crossed. Therefore, a modified $\mathbf{v}_{i}$, from which the unphysical, singular piece is removed, is used to construct an elastic theory in the presence of a dislocation. The modified $\mathbf{v}_{i}$ is equal to $\nabla u_{i}$ everywhere except at the cut.

Using Stokes law, Eqs. (7.3a) and (7.3b) can be rewritten in differential form

$$
\boldsymbol{\nabla} \times \mathbf{v}_{i}=\mathbf{m}_{i} .
$$

Since $\mathbf{v}_{i}$ is no longer the gradient of a single-valued field, it can have a nonzero curl. This is where the presence of the dislocation is manifest. In the absence of a dislocation the curl would be zero. The field $\mathbf{m}$ is defined as

$$
\mathbf{m}_{i}(\mathbf{r})=\sum_{j} \int a_{i} N_{j} \mathbf{t}_{j}\left[\mathbf{r}_{j}\left(s_{j}\right)\right] \delta^{3}\left[\mathbf{r}-\mathbf{r}_{i}\left(s_{j}\right)\right] d s_{j},
$$

where $s_{j}$ parametrizes the $j$ th dislocation loop, $\mathbf{r}_{j}\left(s_{j}\right)$ is the position of that loop, $\mathbf{t}_{j}\left[\mathbf{r}_{j}\left(s_{j}\right)\right]$ is its local unit tangent, and $N_{j}$ the "charge" or number of excess lattice vectors along $\hat{\mathbf{a}}_{i}$ associated with the dislocation. Note that $N_{j}$ is independent of $s_{j}$, since the charge of a given line is constant along the line defect. It is important to note that $\mathbf{v}_{i}$ is not the $i$ th component of the three-dimensional vector $\mathbf{v}$. Rather, it is one of the two vectors $\mathbf{v}_{1}$ and $\mathbf{v}_{2}$, constructed using Eq. (7.4). The same applies to $\mathbf{m}_{i}$.

Equation (7.5) implies that

$$
\boldsymbol{\nabla} \cdot \mathbf{m}_{i}(\mathbf{r})=0,
$$

which simply means that dislocation lines cannot end in the bulk of the sample; they must either form closed loops or extend entirely through the system. It is useful to work with Fourier transformed fields

$$
\begin{gathered}
\mathbf{v}_{i}(\mathbf{q})=i \mathbf{q} u_{i}(\mathbf{q}), \\
\mathbf{m}_{i}(\mathbf{q})=i \mathbf{q} \times \mathbf{v}_{i}(\mathbf{q}),
\end{gathered}
$$

and work with the longitudinal and transverse components $\mathbf{v}_{L}(\mathbf{q})$ and $\mathbf{v}_{T}(\mathbf{q})$, defined by

$$
\begin{aligned}
& \mathbf{v}_{L}(\mathbf{q})=i \mathbf{q} u_{L}(\mathbf{q}), \\
& \mathbf{v}_{T}(\mathbf{q})=i \mathbf{q} u_{T}(\mathbf{q}),
\end{aligned}
$$

where $u_{L}(\mathbf{q})$ and $u_{T}(\mathbf{q})$ are the longitudinal and transverse components of $\mathbf{u}(\mathbf{q})$ given, in $d=3$, by

$$
\mathbf{u}(\mathbf{q})=u_{L}(\mathbf{q}) \hat{\mathbf{q}}_{\perp}+u_{T}(\mathbf{q})\left(\hat{\mathbf{z}} \times \hat{\mathbf{q}}_{\perp}\right) .
$$

The field $\mathbf{v}_{i}$ can be easily constructed from $\mathbf{v}_{L}(\mathbf{q})$ and $\mathbf{v}_{T}(\mathbf{q})$, according to

$$
\mathbf{v}_{i}(\mathbf{q})=v_{L}(\mathbf{q})\left(\hat{\mathbf{q}}_{\perp}\right)_{i}+v_{T}(\mathbf{q})\left(\hat{\mathbf{z}} \times \hat{\mathbf{q}}_{\perp}\right)_{i},
$$

where $\left(\hat{\mathbf{q}}_{\perp}\right)_{i}$ is the $i$ th component of $\hat{\mathbf{q}}_{\perp}$, and $\left(\hat{\mathbf{z}} \times \hat{\mathbf{q}}_{\perp}\right)_{i}$ is the $i$ th component of $\hat{\mathbf{z}} \times \hat{\mathbf{q}}_{\perp}$. Similarly,

$$
\mathbf{m}_{i}(\mathbf{q})=m_{L}(\mathbf{q})\left(\hat{\mathbf{q}}_{\perp}\right)_{i}+m_{T}(\mathbf{q})\left(\hat{\mathbf{z}} \times \hat{\mathbf{q}}_{\perp}\right)_{i} .
$$

In Fourier space the condition that dislocation lines must form closed loops or extend entirely through the sample, Eq. (7.7) gives

$$
\begin{aligned}
& \mathbf{q} \cdot \mathbf{m}_{L}(\mathbf{q})=0, \\
& \mathbf{q} \cdot \mathbf{m}_{T}(\mathbf{q})=0,
\end{aligned}
$$

and the boundary condition, Eq. (7.5) corresponding to a topological defect is

$$
\begin{aligned}
& i \mathbf{q} \times \mathbf{v}_{L}(\mathbf{q})=\mathbf{m}_{L}(\mathbf{q}), \\
& i \mathbf{q} \times \mathbf{v}_{T}(\mathbf{q})=\mathbf{m}_{T}(\mathbf{q}) .
\end{aligned}
$$

The real-space Hamiltonian to harmonic order in $d=3$ dimensions is given by Eq. (5.3), which we rewrite here for definiteness

$$
H[\mathbf{u}(\mathbf{r})]=\int d \mathbf{r} \frac{1}{2}\left[\left(\lambda v_{\alpha \alpha}^{2}+2 \mu v_{\alpha \beta}^{2}+\kappa\left|\partial_{z}^{2} \mathbf{u}\right|^{2}\right)+\mathbf{h}(\mathbf{r}) \cdot \partial_{z} \mathbf{u}\right],
$$

where the reader is reminded that $\mathbf{h}(\mathbf{r})$ is the random-tilt disorder, with short-range, isotropic correlations

$$
\overline{h_{\alpha}(\mathbf{r}) h_{\beta}\left(\mathbf{r}^{\prime}\right)}=\Delta_{t} \delta_{\alpha \beta} \delta\left(\mathbf{r}-\mathbf{r}^{\prime}\right) .
$$

The above real-space Hamiltonian can then be rewritten in Fourier space, where $\mathbf{u}(\mathbf{q})$ and $\mathbf{h}(\mathbf{q})$ can both be decomposed 
into longitudinal and transverse pieces according to Eq. (7.10). The longitudinal and transverse modes decouple, and one obtains

$$
H[\mathbf{u}(\mathbf{q}), \mathbf{h}(\mathbf{q})]=H_{L}\left[u_{L}(\mathbf{q}), h_{L}(\mathbf{q})\right]+H_{T}\left[u_{T}(\mathbf{q}), h_{T}(\mathbf{q})\right],
$$

with

$$
\begin{aligned}
H_{L / T}\left[u_{L / T}(\mathbf{q}), h_{L / T}(\mathbf{q})\right]= & \frac{1}{2} \sum_{\mathbf{q}}\left[B_{L / T}\left(q_{\perp}^{2}+\lambda_{L / T}^{2} q_{z}^{4}\right)\left|u_{L / T}(\mathbf{q})\right|^{2}\right. \\
& \left.-i q_{z} u(\mathbf{q}) h_{L / T}(-\mathbf{q})\right],
\end{aligned}
$$

where $B_{L}=\lambda+2 \mu, B_{T}=\mu$, and $\lambda_{L / T}^{2}=\kappa / B_{L / T}$. This Hamiltonian does not have any anomalous elasticity in it. It is instructive to perform the dislocation theory at the harmonic level first and then to generalize to a full anharmonic theory afterward.

Using the decoupled Hamiltonians for $u_{L}(\mathbf{q})$ and $u_{T}(\mathbf{q})$ it is possible, using the constraints Eqs. (7.13a), (7.13b), (7.14a), and (7.14b) to obtain decoupled elastic Hamiltonians for the dislocation fields $\mathbf{m}_{L}(\mathbf{q})$ and $\mathbf{m}_{T}(\mathbf{q})$. The first step is to minimize $H_{L / T}\left[u_{L / T}(\mathbf{q}), h_{L / T}(\mathbf{q})\right]$ with respect to $u_{L / T}(\mathbf{q})$. The Euler-Lagrange equation for $u_{L / T}(\mathbf{q})$ is given by

$$
\left(q_{\perp}^{2}+\lambda_{L / T}^{2} q_{z}^{4}\right) u_{L / T}(\mathbf{q})-\frac{i q_{z} h_{L / T}(\mathbf{q})}{B_{L / T}}=0,
$$

and can be rewritten in terms of $\mathbf{v}_{L / T}(\mathbf{q})$ as

$$
\mathbf{q}_{\perp} \cdot \mathbf{v}_{L / T}^{\perp}(\mathbf{q})+\lambda_{L / T}^{2} q_{z}^{3} v_{L / T}^{z}(\mathbf{q})+\frac{q_{z} h_{L / T}(\mathbf{q})}{B_{L / T}}=0,
$$

where $\mathbf{v}_{L / T}^{\perp}(\mathbf{q})$ and $v_{L / T}^{z}(\mathbf{q})$ are the $\perp$ and $z$ components, respectively, of $\mathbf{v}_{L / T}(\mathbf{q})$. The next step is to solve Eq. (7.20), (7.14a), and (7.14b) for $\mathbf{v}_{L / T}(\mathbf{q})$. Doing this, one obtains

$$
\begin{aligned}
\mathbf{v}_{L / T}(\mathbf{q})= & \frac{i \mathbf{q} \times \mathbf{m}_{L / T}(\mathbf{q})}{q^{2}} \\
& +\frac{q_{z} \mathbf{q}}{\Gamma_{q}^{L / T}}\left(\frac{i\left[\mathbf{m}_{L / T}(\mathbf{q})\right]_{z}}{q^{2}}\left(1-\lambda_{L / T}^{2} q_{z}^{2}\right)-\frac{h_{L / T}(\mathbf{q})}{B_{L / T}}\right),
\end{aligned}
$$

where $\Gamma_{q}^{L / T}$ is defined as

$$
\Gamma_{q}^{L / T} \equiv q_{\perp}^{2}+\lambda_{L / T}^{2} q_{z}^{4} .
$$

These expressions for $\mathbf{v}_{L / T}^{\perp}(\mathbf{q})$ can then be inserted back into the original elastic Hamiltonian of Eq. (7.17), which gives the defect Hamiltonian

$$
H_{d}=H_{d}^{L}\left[\mathbf{m}_{L}(\mathbf{q}), h_{L}(\mathbf{q})\right]+H_{d}^{T}\left[\mathbf{m}_{T}(\mathbf{q}), h_{T}(\mathbf{q})\right],
$$

where

$$
\begin{aligned}
H_{d}^{L / T}\left[\mathbf{m}_{L / T}(\mathbf{q}), h_{L / T}(\mathbf{q})\right]= & \sum_{\mathbf{q}}\left[\frac{\kappa q_{z}^{2}\left|\mathbf{m}_{L / T}^{\perp}(\mathbf{q})\right|^{2}+B_{L / T}\left|\mathbf{m}_{L / T}^{z}(\mathbf{q})\right|^{2}}{\Gamma_{q}^{L / T}}\right. \\
& \left.+\mathbf{m}_{L / T}(\mathbf{q}) \cdot \mathbf{a}_{L / T}(-\mathbf{q})\right]
\end{aligned}
$$

and where $\mathbf{a}_{L / T}(\mathbf{q})$ is related to $h_{L / T}(\mathbf{q})$ via

$$
\mathbf{a}_{L / T}(\mathbf{q})=\frac{i h_{L / T}(\mathbf{q})(\hat{\mathbf{z}} \times \mathbf{q})}{\Gamma_{q}^{L / T}} .
$$

\section{B. Duality transformation}

The defect Hamiltonian of Eq. (7.24) makes it possible for us to study the statistical mechanics of the dislocations. We do this by performing a duality transformation. As will be seen below, to perform this transformation it is necessary to have a partition function in which the trace is taken over the real-space dislocation fields. However, as will also be seen below, although this is possible, it is not necessary to rewrite the defect Hamiltonian above [which is expressed in terms of the fields $\left.\mathbf{m}_{L / T}(\mathbf{q})\right]$ in terms of the fields $\mathbf{m}_{1}(\mathbf{q})$ and $\mathbf{m}_{2}(\mathbf{q})$. The reader is reminded that $\mathbf{m}_{1}(\mathbf{r})$ and $\mathbf{m}_{2}(\mathbf{r})$ are the dislocation loop fields associated with dislocations along $\mathbf{a}_{1}$ and $\mathbf{a}_{2}$, respectively, where $\mathbf{a}_{1}$ and $\mathbf{a}_{2}$ are basis vectors of the lattice.

Henceforth, for simplicity, a square lattice will be assumed, i.e., $\mathbf{a}_{1}=a \hat{\mathbf{x}}$ and $\mathbf{a}_{2}=a \hat{\mathbf{y}}$. This assumption makes certain parts of the calculation more straightforward than they would be for arbitrary $\hat{\mathbf{a}}_{1}$ and $\hat{\mathbf{a}}_{2}$, e.g., for a hexagonal lattice. This assumption may seem invalid given that the Hamiltonian which has been used thus far is that of a hexagonal lattice. However, the determination of topological stability is ultimately based on scaling arguments. Since the scaling (e.g., of the propagators and correlation functions) is the same in the hexagonal and square lattices, the answer, which depends only on this scaling, obtained using this innocuous simplification to a square lattice should be the same as that for the hexagonal lattice. This assumption would not, however, be so innocuous for a theory of the transition, where the structure of the lattice is important.

The first step in the duality transformation is to put the model on a simple cubic lattice (to make it well-defined at short distances); now, $\mathbf{m}_{1}(\mathbf{r})$ and $\mathbf{m}_{2}(\mathbf{r})$ are defined on the sites $\mathbf{r}$ of the lattice, and they take on values

$$
\mathbf{m}_{i}(\mathbf{r})=\frac{a_{i}}{d^{2}}\left(n_{i}^{x}(\mathbf{r}), n_{i}^{y}(\mathbf{r}), n_{i}^{z}(\mathbf{r})\right),
$$

where $n_{i}^{\alpha}$ is the $\alpha$ th component of $\mathbf{n}_{i}$ and the Einstein summation convention has been suspended. Each of $n_{i}^{x}, n_{i}^{y}$, and $n_{i}^{z}$ is an integer. For the square lattice considered here the lengths of the two basis vectors are the same so that $a_{1}=a_{2}$ $=a$. $d$ is the cubic lattice constant used in the discretization. The partition function for this model is then

$$
Z[\{\mathbf{h}\}]=\sum_{i} \sum_{\left\{\mathbf{m}_{i}(\mathbf{r})\right\}}^{\prime} e^{-S\left[\left\{\mathbf{m}_{i}\right\}\right]},
$$

where

$$
S\left[\left\{\mathbf{m}_{i}\right\}\right] \equiv \beta\left[H_{d}\left[\left\{\mathbf{m}_{1}, \mathbf{m}_{2}\right\}\right]+E_{c} \sum_{\mathbf{r}, i}\left|\mathbf{m}_{i}(\mathbf{r})\right|^{2}\right],
$$

and the sum is over all integer-valued configurations of $\mathbf{m}_{i}{ }^{\text {'s }}$ given by Eq. (7.26), satisfying the dislocation line continuity constraint, which is denoted by the prime,

$$
\nabla \cdot \mathbf{m}_{i}=0,
$$


where the divergence now represents a lattice divergence, $H_{d}$ is obtainable from Eq. (7.24), and a core energy term $E_{c} \Sigma_{\mathbf{r}, i}\left|\mathbf{m}_{i}(\mathbf{r})\right|^{2}$ has been added, to account for energies near the cores of the defect line that are not accurately treated by the above continuum elastic theory. The symmetry of the square lattice dictates that the core energies be the same for both $\mathbf{m}_{1}(\mathbf{r})$ and $\mathbf{m}_{2}(\mathbf{r})$. The reader's attention is directed to the fact that the partition function still depends implicitly on the configuration of the random fields $\{\mathbf{h}\}$.

To proceed, the constraints $\nabla \cdot \mathbf{m}_{1}=0$ and $\nabla \cdot \mathbf{m}_{2}=0$ are enforced by introducing auxiliary fields $\theta_{1}(\mathbf{r})$ and $\theta_{2}(\mathbf{r})$ and rewriting the partition function [Eq. (7.27)] as

$$
Z=\prod_{\mathbf{r}} \int d \theta_{1}(\mathbf{r}) d \theta_{2}(\mathbf{r}) \sum_{i} \sum_{\left\{\mathbf{m}_{i}(\mathbf{r})\right\}} e^{-S\left[\left\{\mathbf{m}_{i}\right\}\right]+i \Sigma_{\mathbf{r}, i} \theta_{i}(\mathbf{r}) \nabla \cdot \mathbf{m}_{i}(\mathbf{r}) d^{2} / a},
$$

where the sum over $\left\{\mathbf{m}_{i}\right\}$ is now unconstrained (and no longer has a prime), the constraint $\nabla \cdot \mathbf{m}_{i}=0$ is enforced by integration over $\theta_{i}$, since

$$
\delta\left(\nabla \cdot \mathbf{m}_{i}\right)=\int_{0}^{2 \pi} d \theta_{i}(\mathbf{r}) e^{i \theta_{i}(\mathbf{r}) \nabla \cdot \mathbf{m}_{i}(\mathbf{r}) d^{2} / a},
$$

where the $\delta$ is a Kronecker $\delta$ since $\mathbf{m}_{i}$, and, hence, $\nabla \cdot \mathbf{m}_{i}$, is integer valued.

Now one can "integrate" (actually sum) by parts and rewrite

$$
\sum_{\mathbf{r}, i} \theta_{i}(\mathbf{r}) \nabla \cdot \mathbf{m}_{i}(\mathbf{r})=-\sum_{\mathbf{r}, i} \mathbf{m}_{i}(\mathbf{r}) \cdot \nabla \theta_{i}(\mathbf{r})+\text { surface terms } .
$$

The next step is to introduce dummy gauge fields $\mathbf{A}_{1}$ and $\mathbf{A}_{2}$ to mediate the long-range interaction between the defect loops $\mathbf{m}_{1}$ and $\mathbf{m}_{2}$ in the Hamiltonian equation (7.23). This is accomplished using the Hubbard-Stratonovich transformation and rewriting the partition function as

$$
\begin{aligned}
Z= & \prod_{\mathbf{r}} \int d \theta_{1}(\mathbf{r}) d \theta_{2}(\mathbf{r}) d \mathbf{A}_{1}(\mathbf{r}) d \mathbf{A}_{2}(\mathbf{r}) \\
& \times \sum_{i} \sum_{\left\{\mathbf{m}_{i}(\mathbf{r})\right\}} e^{-S\left[\left\{\mathbf{m}_{i}\right\}, \theta_{i}, \mathbf{A}_{i}\right]} \delta\left(\nabla \cdot \mathbf{A}_{i}\right),
\end{aligned}
$$

with

$$
\begin{aligned}
S= & \frac{1}{T} \sum_{\mathbf{r}, i}\left\{\mathbf{m}_{i}(\mathbf{r}) \cdot\left[-i \frac{T d^{2}}{a} \boldsymbol{\nabla} \theta_{i}(\mathbf{r})+d^{3}\left[i \mathbf{A}_{i}(\mathbf{r})+a_{i}(\mathbf{r})\right]\right]\right. \\
& \left.+E_{c}\left|\mathbf{m}_{i}\right|^{2}\right\}+\frac{1}{2} \sum_{\mathbf{q}}\left[\frac{\Gamma_{q}^{L}}{\kappa q_{z}^{2}}\left|\mathbf{A}_{L}^{\perp}(\mathbf{q})\right|^{2}+\frac{\Gamma_{q}^{L}}{B_{L}}\left|\mathbf{A}_{L}^{z}(\mathbf{q})\right|^{2}\right. \\
& \left.+\frac{\Gamma_{q}^{T}}{\kappa q_{z}^{2}}\left|\mathbf{A}_{T}^{\perp}(\mathbf{q})\right|^{2}+\frac{\Gamma_{q}^{T}}{B_{T}}\left|\mathbf{A}_{T}^{z}(\mathbf{q})\right|^{2}\right],
\end{aligned}
$$

where $\mathbf{A}_{L}(\mathbf{q})$ and $\mathbf{A}_{T}(\mathbf{q})$ are defined in the same way as $\mathbf{v}_{L / T}(\mathbf{q})$ and $\mathbf{m}_{L / T}(\mathbf{q})$, i.e.,

$$
\mathbf{A}_{L}(\mathbf{q})=\hat{\mathbf{q}}_{1} \mathbf{A}_{1}(\mathbf{q})+\hat{\mathbf{q}}_{2} \mathbf{A}_{2}(\mathbf{q}),
$$

$$
\mathbf{A}_{T}(\mathbf{q})=(\hat{\mathbf{z}} \times \hat{\mathbf{q}})_{1} \mathbf{A}_{1}(\mathbf{q})+(\hat{\mathbf{z}} \times \hat{\mathbf{q}})_{2} \mathbf{A}_{1}(\mathbf{q}),
$$

where $\left(\hat{\mathbf{q}}_{\perp}\right)_{i}$ is the $i$ th component of $\hat{\mathbf{q}}_{\perp}$ and $\left(\hat{\mathbf{z}} \times \hat{\mathbf{q}}_{\perp}\right)_{i}$ is the $i$ th component of $\hat{\mathbf{z}} \times \hat{\mathbf{q}}_{\perp}$, and where $\mathbf{a}_{i}(\mathbf{r})$ is the Fourier transform of

$$
\mathbf{a}_{i}(\mathbf{q})=\mathbf{a}_{L}(\mathbf{q})\left(\hat{\mathbf{q}}_{\perp}\right)_{i}+\mathbf{a}_{T}(\mathbf{q})\left(\hat{\mathbf{z}} \times \hat{\mathbf{q}}_{\perp}\right)_{i} .
$$

The two goals of all these manipulations have now been achieved: the sum on $\left\{\mathbf{m}_{i}(\mathbf{r})\right\}$ is now unconstrained and the sum on each site over $\mathbf{m}_{i}(\mathbf{r})$ is now decoupled from that on every other site. Furthermore, this sum is readily recognized to be nothing more than the "periodic Gaussian" first used by Villain. ${ }^{38}$ The partition function [Eq. (7.33)] can thus be rewritten

$$
\begin{aligned}
Z= & \prod_{\mathbf{r}} \int d \theta_{1}(\mathbf{r}) d \theta_{2}(\mathbf{r}) d \mathbf{A}_{1}(\mathbf{r}) d \mathbf{A}_{2}(\mathbf{r}) \delta\left[\nabla \cdot \mathbf{A}_{1}(\mathbf{r})\right] \delta\left[\nabla \cdot \mathbf{A}_{1}(\mathbf{r})\right] \\
& \times \exp \left\{-\sum_{\mathbf{r}, i, \alpha} V_{p}\left[\theta_{i}\left(\mathbf{r}+\hat{\mathbf{x}}_{\alpha}\right)-\theta_{i}(\mathbf{r})-A_{i}^{\alpha}(\mathbf{r})+i a_{i}^{\alpha}(\mathbf{r})\right]\right. \\
& -\frac{1}{2} \sum_{\mathbf{q}}\left[\frac{\Gamma_{q}^{L}}{\kappa q_{z}^{2}}\left|\mathbf{A}_{L}^{\perp}(\mathbf{q})\right|^{2}+\frac{\Gamma_{q}^{L}}{B_{L}}\left|\mathbf{A}_{L}^{z}(\mathbf{q})\right|^{2}+\frac{\Gamma_{q}^{T}}{\kappa q_{z}^{2}}\left|\mathbf{A}_{T}^{\perp}(\mathbf{q})\right|^{2}\right. \\
& \left.\left.+\frac{\Gamma_{q}^{T}}{B_{T}}\left|\mathbf{A}_{T}^{z}(\mathbf{q})\right|^{2}\right]\right\},
\end{aligned}
$$

where $A_{i}^{\alpha}(\mathbf{r})$ is the $\alpha$ th component of $\mathbf{A}_{i}(\mathbf{r})$ and the wellknown $2 \pi$-periodic Villain potential $V_{p}(x)$

$$
e^{-V_{p}(x)} \equiv \sum_{n=-\infty}^{\infty} e^{-n^{2} E_{c} / T+i x n}
$$

has the usual property that the smaller $E_{c} / T$ is (i.e., the higher the temperature in the original random-tilt smectic model), the sharper the potential minima. This can be seen by looking at two extremes. When $E_{c} / T$ is zero, the Villain potential is a just a periodic set of $\delta$ functions. When it is very large, then the $n=0, \pm 1$ terms of the series dominate and the potential is a constant plus a cosine of $x$, which is obviously not as sharp as a set of $\delta$ delta functions. Furthermore, the amplitude of the cosine is proportional to $e^{-E_{c} / T}$ and hence gets smaller as $t$ is reduced. Thus, raising the temperature in the original model is like lowering the temperature in the dual model [Eq. (7.37)]. It is precisely this familiar temperature inversion associated with duality that leads to an inverted XY transition for bulk smectics ${ }^{55}$ and threedimensional disorder-free charged superfluids. ${ }^{56}$ It also plays an important role here, as we shall soon see.

Standard universality arguments imply that replacing the periodic potential $V_{p}(x)$ in Eq. (7.37) by any other nonsingular periodic function should not change the universality class of the transition. In particular, we could replace $V_{p}(x)$ by $\cos (x)$. The resultant model would be precisely the "fixedlength" version of the "soft spin," or Landau-GinsburgWilson model, with the complex "action" 


$$
\begin{aligned}
S= & \sum_{\{\mathbf{r}\}}\left\{\frac{c}{2}\left[\nabla+\frac{a d}{T}\left(i \mathbf{A}_{i}+\mathbf{a}_{i}\right)\right] \psi_{i} \cdot\left[\nabla-\frac{a d}{T}\left(i \mathbf{A}_{i}+\mathbf{a}_{i}\right)\right] \psi_{i}^{*}\right. \\
& \left.+t\left|\psi_{i}\right|^{2}+u\left|\psi_{i}\right|^{4}+w\left|\psi_{1}\right|^{2}\left|\psi_{2}\right|^{2}\right\}+\frac{1}{2} \sum_{\mathbf{q}}\left[\frac{\Gamma_{q}^{L}}{\kappa q_{z}^{2}}\left|\mathbf{A}_{L}^{\perp}(\mathbf{q})\right|^{2}\right. \\
& \left.+\frac{\Gamma_{q}^{L}}{B_{L}}\left|\mathbf{A}_{L}^{z}(\mathbf{q})\right|^{2}+\frac{\Gamma_{q}^{T}}{\kappa q_{z}^{2}}\left|\mathbf{A}_{T}^{\perp}(\mathbf{q})\right|^{2}+\frac{\Gamma_{q}^{T}}{B_{T}}\left|\mathbf{A}_{T}^{z}(\mathbf{q})\right|^{2}\right],
\end{aligned}
$$

where $\psi_{i}(\mathbf{r})$ is a complex field whose phase is $\theta_{i}(\mathbf{r})$, and the reduced temperature $t$ and quartic coupling $u$ are parameters of the model [and are the same for $\psi_{1}(\mathbf{r})$ and $\psi_{2}(\mathbf{r})$ because of the symmetry of the square lattice]. Because of the duality transformation's inversion of the temperature axis, the reduced temperature $t$ is a monotonically decreasing function of the temperature $T$ (of the original dislocation loop model), which vanishes at the mean-field transition temperature $T_{M F}$ of the fixed-length model [Eq. (7.37)].

Universality also implies that this soft-spin model should be in the same universality class as the fixed-length model [Eq. (7.37)]. Therefore Eq. (7.39), shall henceforth be used because it is more straightforward to analyze perturbatively.

Before undertaking that analysis, it is worth noting another consequence of duality inversion of the temperature axis: the ordered phase of the dual model [Eq. (7.39)] corresponds to the disordered (i.e., dislocation loops unbound) phase of the original dislocation loop gas model. That is, the low dual-temperature phase described by

$$
\langle\psi(\mathbf{r})\rangle \neq 0
$$

corresponds to the topologically disordered, dislocation unbound phase of the SV lattice.

\section{Analysis of the dual model}

Disorder is included in Eq. (7.39) through the quenched gauge fields $\mathbf{a}_{1}(\mathbf{r})$ and $\mathbf{a}_{2}(\mathbf{r})$, which are related to the random tilt field $\mathbf{h}(\mathbf{r})$ by Eqs. (7.25)-(7.36). The partition function

$$
\begin{aligned}
Z[\{\mathbf{h}\}]= & \int\left[d \psi_{1} d \psi_{2}\right]\left[d \mathbf{A}_{1} d \mathbf{A}_{2}\right] e^{-S\left[\psi_{i}, \mathbf{A}_{i}, \mathbf{h}\right]} \\
& \times \delta\left(\nabla \cdot \mathbf{A}_{1}\right) \delta\left(\nabla \cdot \mathbf{A}_{2}\right)
\end{aligned}
$$

with $S$ given by Eq. (7.39), is thus an implicit function of the random tilt field configuration $\{\mathbf{h}(\mathbf{r})\}$.

The dependence of the partition function $Z$ on the quenched field $\{\mathbf{h}\}$ is dealt with by using the replica trick [Eq. (5.9)]. Doing this leads to the replicated partition function

$$
\begin{aligned}
\overline{Z^{n}}= & \int\left[d \mathbf{a}_{1}\right]\left[d \mathbf{a}_{2}\right] \prod_{a=1}^{n}\left[d \psi_{1 a}\right]\left[d \psi_{2 a}\right]\left[d \mathbf{A}_{1 a}\right]\left[d \mathbf{A}_{2 a}\right] \\
& \times e^{-S_{r}\left[\psi_{i a}, \mathbf{A}_{i a}, \mathbf{a}_{i}\right]} P\left[\mathbf{a}_{1}\right] P\left[\mathbf{a}_{2}\right] \delta\left(\nabla \cdot \mathbf{A}_{1}\right) \delta\left(\nabla \cdot \mathbf{A}_{2}\right),
\end{aligned}
$$

with

$$
\begin{aligned}
S_{r}= & \sum_{\mathbf{r}} \sum_{a=1}^{n}\left\{\frac{c}{2}\left[\boldsymbol{\nabla}+\frac{a d}{T}\left(i \mathbf{A}_{i a}+\mathbf{a}_{i}\right)\right] \psi_{i a}\right. \\
& \times\left[\boldsymbol{\nabla}-\frac{a d}{T}\left(i \mathbf{A}_{i a}+\mathbf{a}_{i}\right)\right] \psi_{i a}^{*}+t\left|\psi_{i a}\right|^{2} \\
& \left.+u\left|\psi_{i a}\right|^{4}+w\left|\psi_{1 a}\right|^{2}\left|\psi_{2 a}\right|^{2}\right\}+\frac{1}{2} \sum_{\mathbf{q}}\left[\frac{\Gamma_{q}^{L}}{\kappa q_{z}^{2}}\left|\mathbf{A}_{L a}^{\perp}(\mathbf{q})\right|^{2}\right. \\
& \left.+\frac{\Gamma_{q}^{L}}{B_{L}}\left|\mathbf{A}_{L a}^{z}(\mathbf{q})\right|^{2}+\frac{\Gamma_{q}^{T}}{\kappa q_{z}^{2}}\left|\mathbf{A}_{T a}^{\perp}(\mathbf{q})\right|^{2}+\frac{\Gamma_{q}^{T}}{B_{T}}\left|\mathbf{A}_{T a}^{z}(\mathbf{q})\right|^{2}\right]
\end{aligned}
$$

Note that $\mathbf{A}_{1 a}$ is the $a$ th replica of the $\mathbf{A}_{1}$ field, not the $a$ th component of $\mathbf{A}_{1}$. The probability distribution $P\left[\mathbf{a}_{i}\right]$ of the field $\mathbf{a}_{i}$ in Eq. (7.42) is Gaussian, defined by Eq. (7.16). Thus the distributions of $P\left[\mathbf{a}_{1}\right]$ and $P\left[\mathbf{a}_{2}\right]$ are completely specified by the averages $\overline{a_{1}^{i}(\mathbf{q}) a_{1}^{j}(-\mathbf{q})}$ and $\overline{a_{2}^{i}(\mathbf{q}) a_{2}^{j}(-\mathbf{q})}$, respectively. These are easily evaluated using the relations (7.25) and (7.36). For $\mathbf{a}_{1}$,

$$
\begin{aligned}
\overline{a_{1}^{i}(\mathbf{q}) a_{1}^{j}(-\mathbf{q})} \\
=\overline{a_{L}^{i}(\mathbf{q}) a_{L}^{j}(-\mathbf{q})}\left[\left(\hat{\mathbf{q}}_{\perp}\right)_{1}\right]^{2}+\overline{a_{T}^{i}(\mathbf{q}) a_{T}^{j}(-\mathbf{q})}\left[\left(\hat{\mathbf{z}} \times \hat{\mathbf{q}}_{\perp}\right)_{1}\right]^{2} \\
\quad+2 \overline{a_{L}^{i}(\mathbf{q}) a_{T}^{j}(-\mathbf{q})}\left(\hat{\mathbf{q}}_{\perp}\right)_{1}\left(\hat{\mathbf{z}} \times \hat{\mathbf{q}}_{\perp}\right)_{1} \\
=\delta_{i j}^{\perp} \Delta_{t} q_{\perp}^{2}\left[\frac{\left[\left(\hat{\mathbf{q}}_{\perp}\right)_{1}\right]^{2}}{\left(\Gamma_{q}^{L}\right)^{2}}+\frac{\left[\left(\hat{\mathbf{z}} \times \hat{\mathbf{q}}_{\perp}\right)_{1}\right]^{2}}{\left(\Gamma_{q}^{T}\right)^{2}}\right]
\end{aligned}
$$

where the second equality was obtained using Eq. (7.16), and where we defined $\delta_{i j}^{\perp}=\delta_{i j}-\delta_{i z} \delta_{j z}$. Similarly,

$$
\overline{a_{2}^{i}(\mathbf{q}) a_{2}^{j}(-\mathbf{q})}=\delta_{i j}^{\perp} \Delta_{t} q_{\perp}^{2}\left[\frac{\left[\left(\hat{\mathbf{q}}_{\perp}\right)_{2}\right]^{2}}{\left(\Gamma_{q}^{L}\right)^{2}}+\frac{\left[\left(\hat{\mathbf{z}} \times \hat{\mathbf{q}}_{\perp}\right)_{2}\right]^{2}}{\left(\Gamma_{q}^{T}\right)^{2}}\right] .
$$

One now must consider the statistical mechanics of the model defined by Eqs. (7.42) through (7.45), in the limit $n$ $\rightarrow 0$.

Since it is only necessary to determine whether the topologically ordered columnar elastic-glass phase is stable against disorder induced dislocation loop unbinding, a complete analysis of the critical properties of the dislocation loop unbinding is unnecessary. Thus, it is sufficient to follow the example of Radzihovsky and Toner ${ }^{29}$ and consider only the limit in which $u \ll 1$. As a result, the $\psi_{i}$ fluctuations are subdominant to those of the gauge field $\mathbf{A}_{i}$ allowing a mean-field treatment of $\psi_{i}$. In this case the remaining gauge fluctuations may be treated exactly. The stability of the columnar elastic glass is assessed by studying the effect of diagrammatic corrections on the reduced dual temperature $t$. The lowest-order contribution to the renormalized dual temperature $t_{R}$ comes from the average of the "diamagnetic" terms

$$
\delta S=\frac{c a^{2} d^{2}}{2 T^{2}} \sum_{i} \sum_{\mathbf{q}}\left(\left\langle\left|\mathbf{A}_{i}\right|^{2}\right\rangle-\overline{\left|\mathbf{a}_{i}\right|^{2}}\right)\left|\psi_{i}\right|^{2},
$$

graphically illustrated in Fig. 6. 


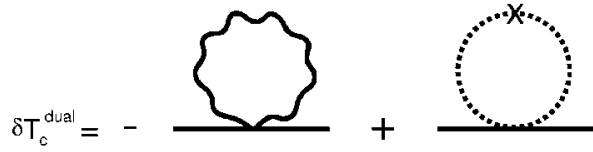

FIG. 6. The Feynman diagrams that dominate the renormalization of the reduced dual temperature $t$.

Taking into account the symmetry of the square lattice and first angular averaging in the $\perp$ plane, the correction to the renormalized reduced dual temperature is, in $d=3$ dimensions,

$$
\begin{aligned}
& t_{R}=t_{0}+\begin{array}{c}
c a^{2} d^{2} \\
T^{2}
\end{array} \int_{q}\left[\kappa q_{z}^{2}\left(\begin{array}{c}
1 \\
\Gamma_{q}^{L}
\end{array}+\begin{array}{c}
1 \\
\Gamma_{q}^{T}
\end{array}\right)+\begin{array}{l}
B_{L} \\
\Gamma_{q}^{L}
\end{array}+\begin{array}{l}
B_{T} \\
\Gamma_{q}^{T}
\end{array}\right. \\
& \left.-\Delta_{t} q_{\perp}^{2}\left(\begin{array}{cc}
1 & 1 \\
\left(\Gamma_{q}^{L}\right)^{2} & + \\
\left(\Gamma_{q}^{T}\right)^{2}
\end{array}\right)\right] \text {, }
\end{aligned}
$$

where the first four terms proportional to $1 / \Gamma_{q}^{L / T}$ come from the first graph in Fig. 6, with the wiggly line representing $\mathbf{A}_{i}$ fluctuations, while the terms proportional to $\Delta_{t}$ come from the second graph with the internal dotted line representing the quenched gauge field $\mathbf{a}_{i}$. The negative sign of the disorder contribution leads to an increase in the dual $T_{c}$ and can be traced back to the fact that the action $S_{r}$ in Eq. (7.43) is complex valued.

Performing this integral, one finds no infrared divergence in spatial dimension $d=3$ (the only dimension in which the analysis of this section applies), indicating that the renormalized $t_{r}$ remains finite as the system size diverges. A finite $t_{r}$ implies that there is a temperature regime in which the dual parameter is in its disordered phase, which, in turn, implies that there is a temperature regime in which dislocations in the columnar phase remain bound. Thus, in the harmonic model, there is a stable columnar elastic glass.

\section{Treating the nonlinearities}

Of course, one would like to also perform a full anharmonic theory of dislocations (since the anharmonic effects are obviously important), but unfortunately such a theory is simply intractable. In particular, the fact that, in an anharmonic theory, the interaction energy between dislocations cannot be written as a sum of pairwise interactions (since their fields do not simply add) makes it impossible to even write down a general expression for the energy of an arbitrary dislocation configuration. At best, one might hope to be able to write down the energy for a few simple, highsymmetry configurations (e.g., a simple, straight dislocation line). Such specialized results would be of no use in a full statistical theory of defect unbinding, which requires consideration of very complicated, tangled configurations of dislocations, which for entropic reasons dominate the free energy near the dislocation unbinding transition.

Furthermore, even if one could write down the anharmonic energy for an arbitrary dislocation configuration, it would presumably be anharmonic in the dislocation fields $\mathbf{m}_{i}(\mathbf{r})$, and hence, those fields would not be decoupled by a simple Hubbard-Stratonovich transformation, as they can in the harmonic model.
For all these reasons, a completely honest treatment of dislocations in the full, anharmonic model is extremely difficult. The next best, tractable (but uncontrolled) approach, introduced by Radzihovsky and Toner, ${ }^{29}$ is to simply replace the elastic constants $\mu, \lambda$, and $\kappa$, and the tilt disorder variance $\Delta_{t}$ in Eq. (7.47) for the renormalized dislocation unbinding transition temperature, with the renormalized wavevector dependent moduli $\mu(\mathbf{q}), \lambda(\mathbf{q}), \kappa(\mathbf{q})$, and $\Delta_{t}(\mathbf{q})$, derived in Sec. VI B. One can hope to justify this procedure by doing an RG matching calculation.

Doing this, simple power counting shows that in the IR limit (i.e., at long length scales) it is the negative, disorder contribution to the renormalization of the dual temperature that dominates, i.e.,

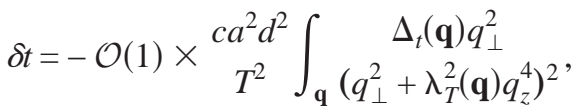

where $\Delta_{t}(\mathbf{q})$ is given by Eq. (6.22) and $\lambda_{T}^{2}(\mathbf{q})=\kappa(\mathbf{q}) / \mu(\mathbf{q})$. The contribution of the piece proportional to $1 /\left(\Gamma_{q}^{L}\right)^{2}$ is absorbed into the $\mathcal{O}(1)$ coefficient, since in the IR limit $\Gamma_{q}^{L}$ and $\Gamma_{q}^{T}$ scale the same way with $\mathbf{q}$ (because $\mu(\mathbf{q}) / \lambda(\mathbf{q})$ is independent of q). Imposing an infrared cutoff $q_{z}>L^{-1}$ on the wave-vector integral in Eq. (7.48), one finds that the infrared piece scales with the linear size of the system $L$ like

$$
\delta t_{I R} \approx-\left(\begin{array}{c}
L \\
\xi_{z}^{N L}
\end{array}\right)^{\gamma} c a^{2} d^{2} \Delta_{t}
$$

with

$$
\gamma=\eta_{\Delta}-1
$$

If $\gamma>0$, the negative correction to $t$ diverges, corresponding to an infinite downward renormalization of the dual temperature, even for arbitrarily weak disorder, implying that dislocations in the SV lattice are always unbound. On the other hand, if $\gamma<0$, then there is no infinite negative divergence of the dual temperature, implying that the columnar elastic glass is stable in a finite range of temperatures.

Using the exact scaling relation in $d=3$, Eq. (6.38) allows $\gamma$ to be reexpressed in terms of the more experimentally accessible $\eta_{\kappa}$ exponent. Doing this one finds

$$
\gamma=2\left(\eta_{\kappa}-1\right)
$$

Thus for $\eta_{\kappa}<1$ the columnar elastic-glass phase is stable. In $d=3$, the estimate of $\eta_{\kappa}$, found in the earlier $\epsilon=7 / 2-d$ expansion, is $\eta_{\kappa}=0.74$, so that a columnar elastic glass is indeed predicted to be stable against dislocation unbinding.

Thus, in summary, for a topologically ordered phase to exist the anomalous exponents must satisfy three distinct criteria, corresponding to a destruction of long-range translational order, the existence of long-range orientational order and stability against dislocation unbinding. These three criteria are, respectively,

$$
\begin{aligned}
& \eta_{\mu}+\eta_{\kappa}>0, \\
& \eta_{\mu}+\eta_{\kappa}<2,
\end{aligned}
$$




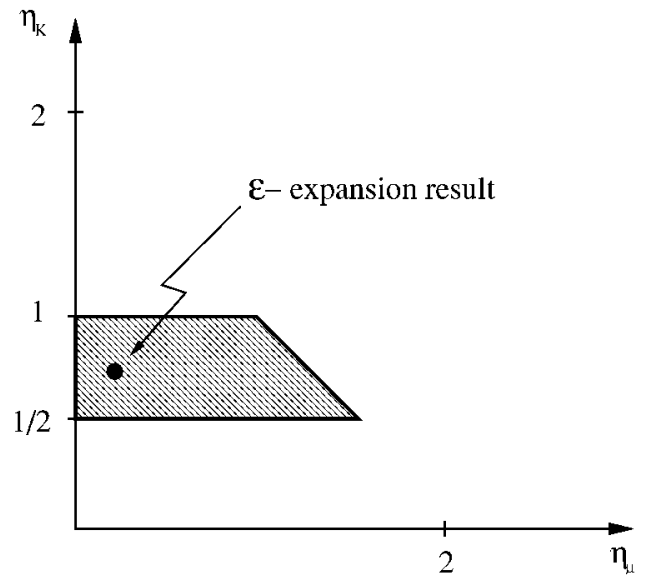

FIG. 7. The region of $\eta_{\kappa}$ and $\eta_{\mu}$ that satisfy the criteria Eqs. (7.52a), (7.52b), and (7.52c) for a long-range orientationally ordered, stable (at sufficiently low disorder strength) columnar elastic-glass phase.

$$
\eta_{\kappa}<1
$$

and are along with a fourth bound that $\eta_{\kappa}>1 / 2$ (which is equivalent, via the exact scaling relation in $d=3$, to the condition that $\eta_{\Delta_{t}}>0$ ), summarized graphically, in Fig. 7. The exponents found in the $\epsilon=7 / 2-d$ expansion satisfy all of these bounds; hence, a topologically ordered phase of the SV lattice is possible in three dimensions. We shall term this phase (which is analogous to the Bragg glass phase of vortices in disordered type-II superconductors ${ }^{34}$ ) a columnar elastic glass (CEG) phase.

\section{MAGNETIZATION CURVE AND OTHER PHENOMENOLOGY}

We expect that for length scales longer than $\xi^{N L}$ the strong, power-law anomalous elasticity will manifest itself in the behavior of all physical observables of the SV solid, such as the width of the structure function peak $S(\mathbf{q})$ measured in neutron scattering and the behavior in its tails. Anomalous elasticity also implies that the stress-strain $\left(\sigma-\boldsymbol{\epsilon}_{c}\right)$ relation becomes non-Hookean, i.e., nonlinear, even for arbitrarily small applied stress $\sigma$. (Note here, that $\sigma$ and $\epsilon_{c}$ should not be confused with the Poisson ratio $\sigma_{p}$ and $\epsilon$-expansion parameter, respectively). In this section we will discuss the expected behavior of the magnetization curve and the structure function.

\section{A. Nonlinear magnetization curve}

The origin of the non-Hookean stress-strain behavior can be seen most clearly for a purely compressive stress, $\sigma_{i j}$ $=\sigma \delta_{i j}$, which, as we shall see shortly, arises from an externally applied magnetic field $\mathbf{H}$ along the flux lines. Such a stress adds a term proportional to

$$
-\sigma \nabla \cdot \mathbf{u}
$$

and a term proportional to

$$
\sigma\left(\partial_{z} \mathbf{u}\right)^{2}
$$

(which explicitly breaks rotational invariance) to the original Hamiltonian. The actual values of the dimensionless constants of proportionality are not important and for simplicity we set them equal to unity. In Fourier space, the second, symmetry-breaking term becomes

$$
\sigma q_{z}^{2}|\mathbf{u}(\mathbf{q})|^{2}
$$

and begins to dominate over the $\kappa(\mathbf{q}) q_{z}^{4}|\mathbf{u}(\mathbf{q})|^{2}$ term once

$$
\sigma q_{z}^{2} \geqslant \kappa(\mathbf{q}) q_{z}^{4}
$$

This clearly happens for $q_{z}$ 's smaller than a critical $q_{c}$ given by

$$
\sigma q_{c}^{2}=\kappa\left(q_{z}=q_{c}, \mathbf{q}_{\perp}=0\right) q_{c}^{4} .
$$

Using Eq. (6.20) for $\kappa(\mathbf{q})$ in this expression and solving for $q_{c}$, we find

$$
q_{c}(\sigma)=\left(\begin{array}{l}
\sigma \\
\kappa
\end{array}\right)^{1 / \delta}\left(\xi_{z}^{N L}\right)^{\eta_{\kappa} / \delta},
$$

where we defined

$$
\delta=2-\eta_{\kappa}
$$

The important thing to note about this result is that it (singularly) depends on the applied stress $\sigma$.

Now, for $q_{z}>q_{c}(\sigma)$ the stress-induced rotational symmetry-breaking term $\sigma q_{z}^{2}|\mathbf{u}(\mathbf{q})|^{2}$ in the Hamiltonian is negligible (subdominant) relative to the vortex curvature energy $\kappa(\mathbf{q}) q_{z}^{4}|\mathbf{u}(\mathbf{q})|^{2}$. However, on longer length scales, $q_{z}$ $\ll q_{c}(\sigma)$, the $\sigma$ term dominates, suppressing the SV solid fluctuations and hence cutting off the anomalous elasticity. Therefore, the elastic moduli saturate, for $q_{z} \ll q_{c}(\sigma)$, at their values at $q_{z}=q_{c}(\sigma), q_{\perp}=0$, given by

$$
\begin{aligned}
& \mu(\mathbf{q} \rightarrow 0) \rightarrow \mu\left(q_{z}=q_{c}, \mathbf{q}_{\perp}=0\right), \\
& \lambda(\mathbf{q} \rightarrow 0) \rightarrow \lambda\left(q_{z}=q_{c}, \mathbf{q}_{\perp}=0\right),
\end{aligned}
$$

and, hence, both are proportional to $q_{c}^{\eta_{\mu}}$. Since $q_{c}$ is a function of the applied stress $\sigma$, as shown in Eq. (8.6), this implies that the long-wavelength limit of the $2 d$ bulk modulus

$$
\begin{aligned}
C(\sigma) & =\mu\left(q_{c}\right)+\lambda\left(q_{c}\right), \\
& =\mu\left(1+x_{*}\right)\left[q_{c}(\sigma) \xi_{z}^{N L}\right]^{\eta_{\mu}}, \\
& \approx \mu\left[q_{c}(\sigma) \xi_{z}^{N L}\right]^{\eta_{\mu}}, \\
& =\mu\left(\begin{array}{c}
\sigma \\
\sigma_{N L}
\end{array}\right)^{\beta},
\end{aligned}
$$

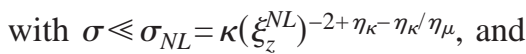

$$
\beta=\stackrel{\eta_{\mu}}{2-\eta_{\kappa}}
$$

To obtain the response of the system to a uniform, isotropic compressive stress within the plane transverse to the 
spontaneous vortex axis, the $2 d$ bulk modulus $C$ times the compressive strain $\epsilon_{c} \equiv \nabla \cdot \mathbf{u}$ must be balanced against the applied compressive stress, i.e., $C(\sigma) \epsilon_{c}=\sigma$. Hence, we find that for a stress $\sigma \leqslant \sigma_{N L}$, the SV glass exhibits nonlinear (non-Hookean) strain-stress relation

$$
\begin{aligned}
\epsilon_{c}(\sigma) & =\sigma / C(\sigma), \\
& \propto \sigma^{\alpha},
\end{aligned}
$$

with

$$
\begin{aligned}
\alpha & =1-\beta, \\
& =1-\frac{\eta_{\mu}}{2-\eta_{\kappa}}, \\
& \approx 0.72,
\end{aligned}
$$

where in the last equality, the numerical $3 d$ values of $\eta_{\mu}$ and $\eta_{\kappa}$ obtained from the $\epsilon$ expansion have been used.

To see that the non-Hookean stress-strain relation, Eq. (8.11), leads to a nonlinear relation between $B$ and $H$, note that the presence of an external field, $\mathbf{H}$, necessitates the inclusion of an additional -B $\cdot \mathbf{H}$ term in the Hamiltonian. A field $\mathbf{H}$ along the $\hat{\mathbf{z}}$ direction [the direction of the initial magnetic induction $\mathbf{B}(H=0)$ ] will therefore induce an increase in the magnetic induction, $\delta B(H) \equiv B(H)-B(H=0)$. In the vortex lattice state, however, since each flux line carries exactly one flux quantum $\phi_{0}$, the only way to increase $B$ is to move the vortex lines closer together, i.e., compress the lattice. This implies that the external field leads to a compressive strain term, proportional to $-H \nabla \cdot \mathbf{u}$. The external field $\mathbf{H}$ also explicitly breaks rotational symmetry, leading to the addition of a term $\sim H\left|\partial_{z} \mathbf{u}\right|^{2}$. Thus, the application of a field $\mathbf{H}$ leads to a compressional strain (proportional to $\delta B$ ) and to explicit rotational symmetry breaking, just as a uniform compressive stress does. The two cases are exactly analogous, with $\sigma$ replaced $^{57}$ by $H$, and the non-Hookean stress-strain relation [Eq. (8.11)] immediately translates into a nonlinear $\delta B(H)-H$ relation of exactly the same form; i.e., into

$$
\delta B(H) \propto H^{\alpha},
$$

with the universal exponent $\alpha=0.72 \pm 0.04$. This relation is shown in Fig. 1.

Now, of course, in a real crystalline ferromagnet, there are always crystalline symmetry-breaking fields, which pick out a preferred direction for the magnetization, thereby explicitly breaking the rotational invariance. These symmetry breaking fields lead to a nonzero tilt modulus, i.e., to a term

$$
\frac{1}{2} V_{\times}\left|\partial_{z} \mathbf{u}\right|^{2}
$$

In the Hamiltonian, which cuts off the anomalous elasticity even in the absence of an external field $\mathbf{H}$. Consequently, we expect the predictions of our rotationally invariant theory to break down beyond a crossover length scale

$$
\xi_{z}^{\times}=\left(\frac{\kappa}{V_{\times}}\right)^{1 / \delta}\left(\xi_{z}^{N L}\right)^{-\eta_{\kappa} / \delta} .
$$

If the crystal symmetry-breaking fields are weak, $V_{\times}$will be small and our anomalous elastic theory will be valid for a wide range of length scales $L_{z, \perp}$ satisfying

$$
\xi_{z, \perp}^{N L} \ll L_{z, \perp} \ll \xi_{z, \perp}^{\times} .
$$

For longer length scales the soft anisotropic elasticity of the SV lattice will cross over to the conventional "tension" elasticity of ordinary vortex lattices. This crossover in length scales manifests itself in a crossover value $H_{\times}$for the applied magnetic field $H$ below which the non-Hookean law [Eq. (1.2)] breaks down, to be replaced by a conventional, Hookean relation $\delta B \propto H$ (as illustrated in Fig. 1). The value of the crossover field $H_{\times}$is proportional to the crystal symmetry-breaking field $V_{\times}$.

The order of magnitude of $H_{\times}$is determined by $V_{\times}$, which, in turn, can be estimated from the width of the hysteresis loops for the magnetic superconductor in the spontaneous vortex lattice state as $V_{\times} \sim M_{0} H_{0}$, where $M_{0}$ is the remnant (spontaneous) magnetization and $H_{0}$ is the coercive field. Although we were not able to find any hysteresis data of ferromagnetic superconductors in the literature, we hope that our work will stimulate such experiments as well as other attempts to estimate $H_{\times}$, such as microscopic models and numerical methods.

The presence of the crystal symmetry-breaking fields also makes it experimentally possible to apply the external magnetic field opposite to the direction of the spontaneous magnetization. Although the true ground state clearly has a "spontaneous" magnetization, which is parallel to the externally applied field, this antiparallel configuration is naively metastable if $|\mathbf{H}|<V_{\times}$. Writing $\mathbf{H}=-H \hat{\mathbf{z}}$, we readily see that, when $H$ reaches the metastability limit $V_{\times}$, the $\frac{1}{2} V_{\times}\left|\partial_{z} \mathbf{u}\right|^{2}$ term is exactly canceled by the $-\frac{1}{2} H\left|\partial_{z} \mathbf{u}\right|^{2}$ term arising from the $\mathbf{H} \cdot \mathbf{M}$ interaction. Thus, at precisely this metastable limit, the flux lattice becomes soft in almost exactly the sense we have been discussing throughout this paper. Therefore, we expect anomalous elasticity out to arbitrarily long length scales at this metastable point, even in the presence of crystal symmetry-breaking fields.

Of course, the exact metastability limit can never be reached; as it is approached, the metastable barrier gets smaller and smaller, and before it can be reduced to zero the system will thermally tunnel over the barrier. Thus, it might appear that the point of exact softness can never be reached, and, hence, that there is no regime with anomalous elasticity out to arbitrarily long length scales.

However, this is not necessarily the case. Recently, it has been found ${ }^{32}$ that if the crystal symmetry-breaking field has the right mixture of higher spherical harmonics in its dependence on the orientation of the flux lattice, it is possible for the metastable state to remain metastable right up to the point of perfect softness. In a sense, the metastability actually persists beyond this point; in fact, as we move beyond the point, of perfect softness, the coefficient $D(H)$ of $\left|\partial_{z} \mathbf{u}\right|^{2}$ in the Hamiltonian becomes negative, inducing an instability not to the true ground state with the flux lattice tipped $180^{\circ}$ relative 
to the metastable state, but, rather, to a local minimum very near the original metastable state, which moves continuously away from the old metastable position as we continue to make $D(H)$ more negative. Indeed, this looks very much like the tilting of the molecules away from the layer normals in a smectic- $A$ to smectic- $C$ transition. We will discuss this fascinating transition more in Sec. IX below.

It should also be noted that many of the ferromagnetic superconductor materials are characterized by strong easyplane anisotropy and only much weaker in-plane crystal symmetry-breaking fields. Our theory can easily accommodate such systems by confining fluctuations of the spontaneous vortex lattice to lie in a plane (that we define to be the $x z$ plane) by setting $u_{y}=0$. The symmetry-breaking field $V_{\times}$ then describes the remnant in-plane $(x z)$ anisotropy. The remnant anisotropy and associated $V_{\times}$should be quite small in such layered easy plane materials. In this case the system is in the universality class of the "hybrid columnar Bragg glass" phase described in Ref. 58, which mixes the properties of an " $m=1$ smectic elastic glass" 31 with those of a conventional Bragg glass.

\section{B. Spontaneous vortex lattice structure function}

Even though the neutron-scattering peaks of the SV lattice will be broad (because of the absence of long-range translational order), they will still contain a great deal of information about the anomalous elasticity of the CEG phase. Since, as demonstrated in Sec. VII (see also Ref. 21), the CEG phase possesses long-range orientational order, in single domain, rather than powdered samples, neutron scattering should be possible..$^{59}$ Thus, the anisotropic scaling information, which is usually lost in a powder-averaged x-rayscattering experiment, would be retained, allowing detailed tests of the quantitative predictions for $\eta_{\kappa}, \eta_{\mu}$, and $\eta_{\Delta_{t}}$.

The neutron scattering will have broadened spots in the $\perp$ plane centered at the reciprocal lattice vectors. The intensity of a Bragg spot centered at reciprocal lattice vector $\mathbf{Q}$ is

$$
I(\mathbf{q})=\overline{\left\langle\left|\rho_{\mathbf{Q}}(\mathbf{q})\right|\right\rangle^{2}},
$$

where $\rho_{\mathbf{Q}}(\mathbf{q})$ is the Fourier transform piece of the spatially varying density of vortex lines modulated along $\mathbf{Q}$. For any lattice $\mathbf{u}$ can be defined via

$$
\rho_{\mathbf{Q}}(\mathbf{r})=\left|\rho_{\mathbf{Q}}\right| e^{i \mathbf{Q} \cdot[\mathbf{r}+\mathbf{u}(\mathbf{r})]},
$$

and so the scattering intensity of Eq. (8.17) can be expressed as

$$
\begin{aligned}
I(\mathbf{q}) & =\left|\rho_{\mathbf{Q}}\right|^{2}\left\langle\int_{\mathbf{r}}\left|e^{i \mathbf{Q} \cdot[\mathbf{u}(\mathbf{r})-\mathbf{u}(\mathbf{0})]} e^{i(\mathbf{Q}-\mathbf{q}) \cdot \mathbf{r}}\right|\right\rangle, \\
& =\left|\rho_{\mathbf{Q}}\right|^{2} \int_{\mathbf{r}} e^{i(\mathbf{Q}-\mathbf{q}) \cdot \mathbf{r}} e^{-\overline{\left\langle[\mathbf{Q} \cdot(\mathbf{u}(\mathbf{r})-\mathbf{u}(\mathbf{0}))]^{2}\right\rangle / 2}},
\end{aligned}
$$

where in going from the first to the second line the property of a Gaussian-distributed variable $x,\left\langle e^{i x}\right\rangle=e^{-\left\langle x^{2}\right\rangle / 2}$, has been used. The effect of the $\mathbf{u}$-dependent exponential is to broaden the peak at $\mathbf{Q}$. This exponential can be expressed in terms of the correlation function $C_{\alpha \beta}(\mathbf{r})=\left\langle\left[u_{\alpha}(\mathbf{r})-u_{\alpha}(0)\right]\left[u_{\beta}(\mathbf{r})\right.\right.$ $\left.\left.-u_{\beta}(0)\right]\right\rangle$

$$
e^{-\overline{\left\langle[\mathbf{Q} \cdot(\mathbf{u}(\mathbf{r})-\mathbf{u}(\mathbf{0}))]^{2}\right\rangle / 2}}=e^{-Q_{\alpha} Q_{\beta} C_{\alpha \beta}(\mathbf{r}) / 2} .
$$

From Eq. (8.20) the widths in the $\hat{q}_{z}$ and $\hat{q}_{\perp}$ directions of the broadened peaks can be obtained. The peak widths in the $\hat{q}_{z}$ and $\hat{q}_{\perp}$ directions are defined as the inverse of the values of $z$ and $r_{\perp}$, respectively, at which the exponent in Eq. (8.20) is $\approx 1 / 2$. Using the expressions for $C_{s}\left(\mathbf{r}_{\perp}, 0\right)$ and $C_{s}\left(\mathbf{0}_{\perp}, z\right)$, given by Eqs. (4.26) (with the appropriate wave-vector anomalous elastic constants and disorder variance) the peak widths $\left(\xi_{z}^{X}\right)^{-1}$ and $\left(\xi_{\perp}^{X}\right)^{-1}$ can be obtained by solving

$$
\begin{gathered}
Q^{-2}=\left(\frac{\mu}{\kappa}\right)\left(\frac{\xi_{z}^{X}}{\xi_{z}^{N L}}\right)^{\eta_{\kappa}+\eta_{\mu}}, \\
Q^{-2}=\left(\frac{\mu}{\kappa}\right)\left(\frac{\xi_{\perp}^{X}}{\xi_{\perp}^{N L}}\right)^{\left(\eta_{\kappa}+\eta_{\mu}\right) / \zeta},
\end{gathered}
$$

which gives

$$
\begin{aligned}
& \xi_{z}^{X}=\xi_{z}^{N L}\left(\frac{\kappa}{G^{2} \mu}\right)^{1 /\left(\eta_{\kappa}+\eta_{\mu}\right)}, \\
& \xi_{\perp}^{X}=\xi_{\perp}^{N L}\left(\frac{\kappa}{G^{2} \mu}\right)^{\zeta /\left(\eta_{\kappa}+\eta_{\mu}\right)} .
\end{aligned}
$$

The temperature dependence of $\xi_{z}^{X}$ and $\xi_{\perp}^{X}$ could be used to determine the exponents $\eta_{\kappa}, \eta_{\mu}$, and $\eta_{\Delta_{t}}$ since the bulk $\kappa(T)$, $\mu(T)$ in Eqs. (8.21a) and (8.21b) have temperature dependences that can be extracted from data on bulk materials.

It is also possible to extract information from the scattering in the tails of the peak. By "tails" one means at wave vectors $\mathbf{q}=\mathbf{Q}+\delta \mathbf{q}$ such that

$$
\left(\xi_{\alpha}^{X}\right)^{-1} \ll\left|\delta q_{\alpha}\right| \ll\left(\xi^{N L}\right)^{-1},
$$

where $\alpha$ is either $\perp$ or $z$. In this regime, the scattering

$$
I(\delta \mathbf{q})=\left|\rho_{\mathbf{Q}}\right|^{2} \int_{\mathbf{r}} e^{-i \delta \mathbf{q} \cdot \mathbf{r}} e^{-\overline{\left\langle[\mathbf{Q} \cdot(\mathbf{u}(\mathbf{r})-\mathbf{u}(\mathbf{0}))]^{2}\right\rangle / 2},}
$$

is dominated by $r<(\delta q)^{-1}$. If $\left|\delta q_{\alpha}\right| \gg\left(\xi_{\alpha}^{X}\right)^{-1}$ [the first part of the condition Eq. (8.23)] then the exponent $\overline{\left\langle[\mathbf{Q} \cdot(\mathbf{u}(\mathbf{r})-\mathbf{u}(\mathbf{0}))]^{2}\right\rangle}$ is still small, because $\xi_{\alpha}^{X}$ is by definition the length one must go to before $\overline{\left\langle[\mathbf{Q} \cdot(\mathbf{u}(\mathbf{r})-\mathbf{u}(\mathbf{0}))]^{2}\right\rangle}$ becomes appreciable. This means that the exponential can be expanded to give

$$
I(\delta \mathbf{q}) \sim \int_{\mathbf{r}} e^{-i \delta \mathbf{q} \cdot \mathbf{r}} \overline{\left\langle[\mathbf{Q} \cdot(\mathbf{u}(\mathbf{r})-\mathbf{u}(\mathbf{0}))]^{2}\right\rangle},
$$

which is just the Fourier transform of the quantity between angular brackets at wave vector $\delta \mathbf{q}$. If $\left|\delta q_{\alpha}\right| \ll\left(\xi^{N L}\right)^{-1}$ [the second part of the condition Eq. (8.23)], then at this wave vector the fluctuations will be anomalous and the intensity will be given by 


$$
I(\mathbf{q}) \sim \frac{\Delta_{t}(\delta \mathbf{q}) \delta q_{z}^{2}}{\left[\mu(\delta \mathbf{q}) \delta q_{\perp}^{2}+\kappa(\delta \mathbf{q}) \delta q_{z}^{4}\right]^{2}},
$$

where the longitudinal and transverse pieces of $\overline{\left\langle u_{i}(\mathbf{q}) u_{j}(-q)\right\rangle}$ have, for simplicity, been set equal, since at $\left|\delta q_{\alpha}\right| \ll \xi_{N L}^{1}$, the longitudinal and transverse propagators are essentially the same [since $\lambda(\delta \mathbf{q}) / \mu(\delta \mathbf{q}) \rightarrow$ const]. Thus, by examining the tails of the broadened peaks one could directly observe the anomalous elasticity.

\section{FUTURE THEORETICAL WORK}

There are a number of interesting questions that remain to be investigated experimentally. One of them is the problem touched on in Sec. VIII of applying a magnetic field in the opposite direction to the spontaneous magnetization to cancel off the effects of the crystal field. The state thereby produced, though only metastable, could, in principle, be exactly, rather than approximately, soft. This exact anomalous elasticity is probably not, however, controlled by the columnar elastic glass fixed point we have been discussing up to now. The reason for this is that the CEG fixed point described a model with exact rotation invariance, to all orders in the rotation angle $\theta$. In contrast, at the metastable fixed point of a model with crystal symmetry-breaking fields, we have simply tuned $H$ to cancel off the leading order in $\theta$ (i.e., the $\left.\left|\partial_{z} \mathbf{u}\right|^{2}\right)$ term in the Hamiltonian. The full model, however, is not rotation invariant to all orders in $\theta$, since, for large $\theta$, the crystal symmetry-breaking field has a different dependence on $\theta$ than the $\mathbf{H} \cdot \mathbf{M}$ term (e.g., $\cos (\theta)$ vs $\cos (4 \theta)$ for an underlying cubic crystal). This lower symmetry of the metastable-limit model allows, e.g., the coefficients of the cubic $(\nabla \cdot \mathbf{u})\left|\partial_{z} \mathbf{u}\right|^{2}$ and quartic $\left|\partial_{z} \mathbf{u}\right|^{4}$ nonlinearities in the Hamiltonian to be different from one another and from those of the quadratic $|\nabla \mathbf{u}|^{2}$ terms. This should be contrasted with the columnar elastic glass Hamiltonian, in which these cubic and quartic terms arise only from the square of the nonlinear strain tensor $v_{i j}$ and must, therefore, have the same coefficients (up to known factors of order unity) as each other and the quadratic terms.

A recent study of this problem ${ }^{32}$ has found that the departure of these cubic and quartic coefficients away from equality with the quadratic terms is a relevant perturbation away from the rotationally invariant fixed point to a nonrotationally invariant fixed point. This fixed point exhibits anomalous elasticity with different universal exponents than those given above for the rotationally invariant problem. Further details will be given in a forthcoming publication. ${ }^{32}$

We have focused in this paper exclusively on static, equilibrium properties of (putative) spontaneous vortex lattices in ferromagnetic superconductors in presence of positional and random tilt disorder. Since, in the presence of tilt disorder these lattices are glassy, their dynamics should exhibit all of the interesting slow phenomenology associated with glasses. The interplay of this slow dynamics with the anomalous elasticity we have studied here should make the dynamic behavior even more fascinating. In particular, the dynamics of depinning of these flux lattices, which determines their voltagecurrent $(I-V)$ characteristics, should be quite interesting.
Note added in proof. It has recently been shown ${ }^{60}$ that the elastic theory developed here for spontaneous flux lattices in $s$-wave superconductors also applies, without modification, to superconductors of arbitrary (e.g., $p$-wave) symmetry.

\section{ACKNOWLEDGMENTS}

A.M.E. wishes to thank the Department of Physics, University of Florida, for financial support. L.R. acknowledges support by the NSF under Grants No. DMR-0213918 and DMR-0321848 and by the David and Lucile Packard Foundation. J.T. thanks the Aspen Center for Physics, and the Kavli Institute for Theoretical Physics, Santa Barbara, for their hospitality while a portion of this work was being completed, and D. Belitz and S. Tewari for many valuable discussions.

\section{APPENDIX A: LARGE-SCALE BEHAVIOR OF CORRELATION FUNCTIONS}

In this Appendix, we give some technical details on the solution of the saddle-point equation (4.20) of the replica formalism of Sec. IV B, which we rewrite here for definiteness

$$
\sigma(u) \int \frac{d^{d_{\perp}} \mathbf{q}_{\perp}}{(2 \pi)^{d_{\perp}}} \int_{-\infty}^{\infty} \frac{d q_{z}}{2 \pi} \frac{T Q_{0}^{2}}{\left[\kappa q_{z}^{4}+K q_{\perp}^{2}+[\sigma](u)\right]^{2}}=1,
$$

with $[\sigma](u)=u \sigma(u)-\int_{0}^{u} d v \sigma(v)$. Using the fact that

$$
\int_{-\infty}^{\infty} \frac{d x}{\left(x^{4}+a^{4}\right)^{2}}=\frac{3 \pi \sqrt{2}}{a^{7}}, \quad a>0
$$

we obtain

$$
\int_{-\infty}^{\infty} \frac{d q_{z}}{\left[\kappa q_{z}^{4}+K q_{\perp}^{2}+[\sigma](v)\right]^{2}}=\frac{3 \pi \sqrt{2}}{8 \kappa^{1 / 4}} \frac{1}{\left[K q_{\perp}^{2}+[\sigma](v)\right]^{7 / 4}},
$$

and hence, Eq. (4.20) becomes

$$
\frac{3 \sqrt{2} T Q_{0}^{2} \sigma(u)}{16 \kappa^{1 / 4}} \int \frac{d^{d}{ }^{\perp} \mathbf{q}_{\perp}}{(2 \pi)^{d_{\perp}}} \frac{1}{\left[K q_{\perp}^{2}+[\sigma](u)\right]^{7 / 4}}=1 .
$$

Performing the change of variables

$$
\mathbf{q}_{\perp}=\mathbf{q} \sqrt{\frac{[\sigma]}{K}},
$$

Eq. (A4) becomes

$$
\frac{3 \sqrt{2} c_{d_{\perp}}}{16 \kappa^{1 / 4} K^{d_{\perp} / 2}} T Q_{0}^{2} \sigma(u)[\sigma(u)]^{\left(2 d_{\perp}-7\right) / 4}=1,
$$

where $c_{d_{\perp}}$ is a numerical constant given by

$$
c_{d_{\perp}}=\int \frac{d^{d_{\perp}} \mathbf{q}_{\perp}}{(2 \pi)^{d_{\perp}}} \frac{1}{\left(q_{\perp}^{2}+1\right)^{7 / 4}},
$$

where, in Eq. (A6), the ultraviolet cutoff $\Lambda=2 \pi / a$ was sent to infinity. Now, Eq. (A5) can be rewritten in the form 


$$
[\sigma(u)]^{\left(7-2 d_{\perp}\right) / 4}=\frac{3 \sqrt{2} c_{d_{\perp}}}{16 \kappa^{1 / 4} K^{d_{\perp} / 2}} T Q_{0}^{2} \sigma(u) .
$$

Taking the derivative of this last expression with respect to $u$ and taking into account the fact that $[\sigma]^{\prime}(u)=u \sigma^{\prime}(u)$, we obtain

$$
\frac{7-2 d_{\perp}}{4}[\sigma(u)]^{\left(3-2 d_{\perp}\right) / 4}=\left(\frac{3 \sqrt{2} c_{d_{\perp}} T Q_{0}^{2}}{16 \kappa^{1 / 4} K^{d_{\perp} / 2}}\right) \frac{1}{u} .
$$

Solving for $[\sigma](u)$, we obtain

$$
[\sigma](u)=\left(\frac{u}{u_{0}}\right)^{2 / \theta},
$$

where we called $\theta=d_{\perp}-3 / 2$ and where $u_{0}$ is given by

$$
u_{0}=\frac{3 \sqrt{2} c_{d_{\perp}} T Q_{0}^{2}}{4\left(7-2 d_{\perp}\right) \kappa^{1 / 4} K^{d_{\perp} / 2}} .
$$

In terms of $[\sigma](u)$, the elastic propagator in the presence of disorder $\tilde{G}(\mathbf{q})$ is given by

$$
\begin{aligned}
\tilde{G}(\mathbf{q}) & =\frac{1}{K q_{\perp}^{2}+\kappa q_{z}^{4}}\left(1+\int_{0}^{1} \frac{d u}{u^{2}} \frac{[\sigma](u)}{K q_{\perp}^{2}+\kappa q_{z}^{4}+[\sigma](u)}\right), \\
& =\frac{1}{K q_{\perp}^{2}+\kappa q_{z}^{4}}\left(1+\int_{0}^{1} \frac{d u}{u^{2}} \frac{\left(u / u_{0}\right)^{2 / \theta}}{K q_{\perp}^{2}+\kappa q_{z}^{4}+\left(u / u_{0}\right)^{2 / \theta}}\right),
\end{aligned}
$$

where, in going from the first to the second line, we replaced $[\sigma](u)$ by its expression [Eq. (A9)]. Now, using the change of variables $x=u^{2 / \theta}$, we can write

$$
\begin{aligned}
\int_{0}^{1} \frac{d u}{u^{2}} \frac{\left(u / u_{0}\right)^{2 / \theta}}{K q_{\perp}^{2}+\kappa q_{z}^{4}+\left(u / u_{0}\right)^{2 / \theta}} \\
\quad=\frac{\theta}{2} \int_{0}^{1} d x \frac{x^{-\theta / 2}}{x+u_{0}^{2 / \theta}\left(K q_{\perp}^{2}+\kappa q_{z}^{4}\right)} \\
\quad=\frac{\theta}{2-\theta} \frac{1}{u_{0}^{2 / \theta}\left(K q_{\perp}^{2}+\kappa q_{z}^{4}\right)} \\
\quad \times{ }_{2} F^{1}\left[1,1-\frac{\theta}{2}, 2-\frac{\theta}{2},-\frac{1}{u_{0}^{2 / \theta}\left(K q_{\perp}^{2}+\kappa q_{z}^{4}\right)}\right],
\end{aligned}
$$

where ${ }_{2} F^{1}$ is a hypergeometric function. Replacing this last expression back into Eq. (A11) and using the following limiting behavior when $a \rightarrow 0$ :

$$
{ }_{2} F^{1}\left(1,1-\frac{\theta}{2}, 2-\frac{\theta}{2},-\frac{1}{a}\right)=\Gamma\left(2-\frac{\theta}{2}\right) \Gamma\left(\frac{\theta}{2}\right) a^{1-(\theta / 2)}+\mathcal{O}(a)
$$

we obtain the following long-wavelength behavior of the correlation function:

$$
\widetilde{G}(\mathbf{q}) \simeq \frac{\theta}{2-\theta} \frac{\Gamma[2-\theta / 2] \Gamma(\theta / 2)}{u_{0}\left(K q_{\perp}^{2}+\kappa q_{z}^{4}\right)^{1+\theta / 2}} .
$$

We now are in a position to calculate the large-scale behavior of the correlation function $C^{p}(\mathbf{r})=\left\langle[\mathbf{u}(\mathbf{r})-\mathbf{u}(0)]^{2}\right\rangle$, which is now given by

$$
C^{p}(\mathbf{r})=\int \frac{d^{d}{ } \mathbf{q}_{\perp} d q_{z}}{(2 \pi)^{d_{\perp}+1}}\left(1-e^{i \mathbf{q} \cdot \mathbf{r}}\right) \tilde{G}(\mathbf{q}),
$$

$$
\begin{aligned}
= & \frac{2 T}{u_{0}} \frac{\theta}{2-\theta} \Gamma(2-\theta / 2) \Gamma(\theta / 2) \\
& \times \int \frac{d^{d}{ }_{\mathbf{q}_{\perp}} d q_{z}}{(2 \pi)^{d_{\perp}+1}} \frac{1-e^{i \mathbf{q} \cdot \mathbf{r}}}{\left(K q_{\perp}^{2}+\kappa q_{z}^{4}\right)^{1+\theta / 2}} .
\end{aligned}
$$

As we did in the last paragraph, we shall evaluate $C^{p}\left(\mathbf{r}_{\perp}\right)$ and $C^{p}(z)$ separately. We have

$$
\begin{aligned}
C^{p}\left(\mathbf{r}_{\perp}\right)= & \frac{2 T}{u_{0} \kappa^{1+\theta / 2}} \frac{\theta}{2-\theta} \Gamma(2-\theta / 2) \Gamma(\theta / 2) \\
& \times \int \frac{d^{d} \mathbf{q}_{\perp}}{(2 \pi)^{d_{\perp}}} \int_{-\infty}^{\infty} \frac{d q_{z}}{2 \pi} \frac{1-e^{i \mathbf{q} \cdot \mathbf{r}}}{\left(q_{z}^{4}+K q_{\perp}^{2} / \kappa\right)^{1+\theta / 2}}, \\
= & \frac{2 T}{\pi u_{0} \kappa^{1+\theta / 2}}\left(\frac{\theta}{2-\theta}\right) \Gamma\left(2-\frac{\theta}{2}\right) \Gamma\left(\frac{\theta}{2}\right) \\
& \times \frac{\Gamma(5 / 4) \Gamma(3 / 4+\theta / 2)}{\Gamma\left(\frac{\kappa}{K}\right)^{(3+2 \theta) / 4}} \\
& \times \int \frac{d^{d_{\perp}}\left(\mathbf{q}_{\perp}\right.}{(2 \pi)^{d_{\perp}}} \frac{1-\mathrm{e}^{i \mathbf{q}_{\perp} \cdot \mathbf{r}_{\perp}}}{q_{\perp}^{(3+2 \theta) / 2}}
\end{aligned}
$$

where, in going from the first to the second line we used the fact that

$$
\int_{-\infty}^{\infty} \frac{d x}{\left(x^{4}+a\right)^{1+(\theta / 2)}}=\frac{2 \Gamma(5 / 4) \Gamma(3 / 4+\theta / 2)}{\Gamma(1+\theta / 2)} a^{-(3 / 4+\theta / 2)} .
$$

For a spontaneous vortex lattice in three dimensions, $d_{\perp}=2$, $\theta=d_{\perp}-3 / 2=1 / 2$, and we obtain

$$
\begin{aligned}
C^{p}\left(r_{\perp}\right) & =\frac{2 T \Gamma(7 / 4) \Gamma(1 / 4)}{3 \pi u_{0} \kappa^{1 / 4} K} \int_{0}^{\Lambda} \frac{d q_{\perp}}{2 \pi} \frac{1-J_{0}\left(q_{\perp} r_{\perp}\right)}{q_{\perp}} \\
& \simeq \frac{T \sqrt{2}}{2 u_{0} \kappa^{1 / 4} K} \ln \left(\Lambda r_{\perp}\right)
\end{aligned}
$$

where, in going from the first to the second line, we used the fact that $\Gamma(7 / 4) \Gamma(1 / 4)=3 \pi \sqrt{2} / 4$.

We now turn our attention to the calculation of $C^{p}(z)$. We have

$$
C^{p}(z)=2 T \int \frac{d^{d_{\perp}} \mathbf{q} d q_{z}}{(2 \pi)^{d_{\perp}+1}}\left(1-e^{i q_{z} z}\right) \tilde{G}(\mathbf{q}),
$$




$$
\begin{aligned}
= & \frac{T}{\pi u_{0} K^{1+\theta / 2}}\left(\frac{\theta}{2-\theta}\right) \Gamma(2-\theta / 2) \Gamma(\theta / 2) \\
& \times \int_{-\infty}^{\infty} d q_{z}\left(1-e^{i q_{z} z}\right) K_{d_{\perp}} \\
& \times \int_{0}^{\Lambda} d q_{\perp} \frac{q_{\perp}^{d_{\perp}-1}}{\left(q_{\perp}^{2}+\kappa q_{z}^{4} / K\right)^{1+\theta / 2}},
\end{aligned}
$$

where $K_{d}=\left[2 \pi^{d / 2} / \Gamma(d / 2)\right]$ is the total solid angle (surface area of a unit sphere) in $d$ dimensions. Now, using the fact that

$$
\begin{aligned}
\int_{0}^{\Lambda} & d q_{\perp} \frac{q_{\perp}^{d_{\perp}-1}}{\left(q_{\perp}^{2}+\kappa q_{z}^{4} / K\right)^{1+\theta / 2}} \\
& =\frac{\Lambda^{d_{\perp}}}{d_{\perp}}\left(\frac{\kappa}{K} q_{z}^{4}\right)^{-(1+\theta / 2)}{ }_{2} F^{1}\left(\frac{d_{\perp}}{2}, 1+\frac{\theta}{2}, 1+\frac{d_{\perp}}{2},-\frac{\Lambda^{2} K}{\kappa q_{z}^{4}}\right),
\end{aligned}
$$

and the limiting behavior when $q_{z} \rightarrow 0$

$$
\begin{aligned}
{ }_{2} F^{1} & \left(\frac{d_{\perp}}{2}, 1+\frac{\theta}{2}, 1+\frac{d_{\perp}}{2},-\frac{\Lambda^{2} K}{\kappa q_{z}^{4}}\right) \\
& \approx \frac{\Gamma\left(1+d_{\perp} / 2\right) \Gamma\left(1+\theta / 2-d_{\perp} / 2\right)}{\Gamma(1+\theta / 2)}\left(\frac{\kappa q_{z}^{4}}{K \Lambda^{2}}\right)^{d_{\perp} / 2},
\end{aligned}
$$

we obtain, in three dimensions $\left(d_{\perp}=2\right)$,

$$
\int_{0}^{\Lambda} d q_{\perp} \frac{q_{\perp}^{d_{\perp}-1}}{\left(q_{\perp}^{2}+\kappa q_{z}^{4} / K\right)^{1+\theta / 2}}=2\left(\frac{K}{\kappa}\right)^{1 / 4} \frac{1}{q_{z}} .
$$

Replacing this last expression into Eq. (A18), we obtain [here we use the fact that $K_{2}=1 /(2 \pi)$ ]

$$
\begin{aligned}
C^{p}(z) & =\frac{2 T \Gamma(7 / 4) \Gamma(1 / 4)}{3 \pi^{2} u_{0} K \kappa^{1 / 4}} \int_{0}^{\Lambda_{z}} d q_{z} \frac{1-\cos \left(q_{z} z\right)}{q_{z}}, \\
& =\frac{T \sqrt{2}}{2 \pi u_{0} K \kappa^{1 / 4}} \int_{0}^{\Lambda_{z} z} d q_{z} \frac{1-\cos (x)}{x},
\end{aligned}
$$

where we introduced the cutoff $\Lambda_{z} \simeq 1 / \xi$ for the integration over $q_{z}$, and where, in going from the first to the second line, we used the change of variables $x=q_{z} z$. Now, from the definition of the cosine-integral function ${ }^{61}$

$$
\operatorname{Ci}(x)=\ln |x|+\gamma+\int_{0}^{x} \frac{\cos t-1}{t} d t,
$$

where $\gamma \simeq 0.577$ is Euler's constant and the fact that $\operatorname{Ci}(x)$ $\rightarrow 0$ when $x \rightarrow \infty$, we see that $\int_{0}^{\Lambda_{z} z} d x(1-\cos x) / x \simeq \ln \left(\Lambda_{z}|z|\right)$ $+\gamma$ for $\Lambda_{z}|z| \gg 1$, and hence the long-distance behavior of $C^{p}(z)$ in three dimensions is given by

$$
C^{p}(z) \simeq \frac{T \sqrt{2}}{2 \pi u_{0} K \kappa^{1 / 4}}[\ln (\Lambda|z|)+\gamma]
$$

as claimed in the text.

\section{APPENDIX B: ELASTIC PROPAGATOR IN THE PRESENCE OF DISORDER}

In this appendix, we show how to derive the expression of the propagator $G_{\alpha \beta}^{a b}(\mathbf{q})$ of the theory defined by the Hamiltonian $H_{0 n}$ of Eq. (5.12),

$$
H_{0 n}=\sum_{a, b} \int_{\mathbf{q}} \frac{1}{2} \Gamma_{\alpha \beta}^{a b}(\mathbf{q}) u_{\alpha}^{a}(\mathbf{q}) u_{\beta}^{b}(-\mathbf{q}),
$$

where

$$
\Gamma_{\alpha \beta}^{a b}(\mathbf{q})=\Gamma_{\alpha \beta}(\mathbf{q}) \delta_{a b}-\frac{\Delta_{t}}{T} q_{z}^{2} \delta_{\alpha \beta}
$$

Finding $G_{\alpha \beta}^{a b}(\mathbf{q})$ amounts to finding the inverse of the matrix $\Gamma_{\alpha \beta}^{a b}(\mathbf{q})$ such that

$$
G_{\alpha \beta}^{a b}(\mathbf{q}) \Gamma_{\beta \gamma}^{b c}(\mathbf{q})=\Gamma_{\alpha \beta}^{a b}(\mathbf{q}) G_{\beta \gamma}^{b c}(\mathbf{q})=\delta_{\alpha \gamma} \delta_{b c} .
$$

It is easy to verify that, in the limit $n \rightarrow 0, G_{\alpha \beta}^{a b}(\mathbf{q})$ is given by

$$
G_{\alpha \beta}^{a b}(\mathbf{q})=T\left(\Gamma^{-1}\right)_{\alpha \beta}(\mathbf{q}) \delta_{a b}+\Delta_{t} q_{z}^{2}\left[\left(\Gamma^{-1}\right)^{2}\right]_{\alpha \beta}(\mathbf{q}) .
$$

But, since

$$
\Gamma_{\alpha \beta}(\mathbf{q})=\Gamma_{L}(\mathbf{q}) P_{\alpha \beta}^{L}\left(\mathbf{q}_{\perp}\right)+\Gamma_{T}(\mathbf{q}) P_{\alpha \beta}^{T}\left(\mathbf{q}_{\perp}\right),
$$

we see that

$$
\left(\Gamma^{-1}\right)_{\alpha \beta}(\mathbf{q})=\Gamma_{L}^{-1}(\mathbf{q}) P_{\alpha \beta}^{L}\left(\mathbf{q}_{\perp}\right)+\Gamma_{T}^{-1}(\mathbf{q}) P_{\alpha \beta}^{T}\left(\mathbf{q}_{\perp}\right),
$$

and

$$
\begin{aligned}
{\left[\left(\Gamma^{-1}\right)^{2}\right]_{\alpha \beta}(\mathbf{q})=} & \left(\Gamma^{-1}\right)_{\alpha \gamma}(\mathbf{q})\left(\Gamma^{-1}\right)_{\gamma \beta}(\mathbf{q}), \\
= & {\left[\Gamma_{L}^{-1}(\mathbf{q}) P_{\alpha \gamma}^{L}\left(\mathbf{q}_{\perp}\right)+\Gamma_{T}^{-1}(\mathbf{q}) P_{\alpha \gamma}^{T}\left(\mathbf{q}_{\perp}\right)\right] } \\
& \times\left[\Gamma_{L}^{-1}(\mathbf{q}) P_{\gamma \beta}^{L}\left(\mathbf{q}_{\perp}\right)+\Gamma_{T}^{-1}(\mathbf{q}) P_{\gamma \beta}^{T}\left(\mathbf{q}_{\perp}\right)\right], \\
= & \Gamma_{L}^{-2}(\mathbf{q}) P_{\alpha \beta}^{L}\left(\mathbf{q}_{\perp}\right)+\Gamma_{T}^{-2}(\mathbf{q}) P_{\alpha \beta}^{T}\left(\mathbf{q}_{\perp}\right),
\end{aligned}
$$

where, in going from the second to the third line, we used the following results from projection operators algebra:

$$
\begin{gathered}
P_{\alpha \gamma}^{L}\left(\mathbf{q}_{\perp}\right) P_{\gamma \beta}^{L}\left(\mathbf{q}_{\perp}\right)=P_{\alpha \beta}^{L}\left(\mathbf{q}_{\perp}\right), \\
P_{\alpha \gamma}^{T}\left(\mathbf{q}_{\perp}\right) P_{\gamma \beta}^{T}\left(\mathbf{q}_{\perp}\right)=P_{\alpha \beta}^{T}\left(\mathbf{q}_{\perp}\right), \\
P_{\alpha \gamma}^{L}\left(\mathbf{q}_{\perp}\right) P_{\gamma \beta}^{T}\left(\mathbf{q}_{\perp}\right)=0 .
\end{gathered}
$$

We, therefore, obtain for $G_{\alpha \beta}^{a b}(\mathbf{q})$, in the limit $n \rightarrow 0$

$$
G_{\alpha \beta}^{a b}(\mathbf{q})=G_{L}^{a b}(\mathbf{q}) P_{\alpha \beta}^{L}\left(\mathbf{q}_{\perp}\right)+G_{T}^{a b}(\mathbf{q}) P_{\alpha \beta}^{T}\left(\mathbf{q}_{\perp}\right),
$$

where

$$
\begin{aligned}
& G_{L}^{a b}(\mathbf{q})=T \Gamma_{L}^{-1}(\mathbf{q}) \delta_{a b}+\Delta_{t} q_{z}^{2} \Gamma_{L}^{-2}(\mathbf{q}), \\
& G_{T}^{a b}(\mathbf{q})=T \Gamma_{T}^{-1}(\mathbf{q}) \delta_{a b}+\Delta_{t} q_{z}^{2} \Gamma_{T}^{-2}(\mathbf{q}) .
\end{aligned}
$$

We, therefore, can write

$$
\left\langle u_{\alpha}^{a}(\mathbf{q}) u_{\beta}^{b}\left(\mathbf{q}^{\prime}\right)\right\rangle_{0}=(2 \pi)^{d} \delta\left(\mathbf{q}+\mathbf{q}^{\prime}\right) G_{\alpha \beta}^{a b}(\mathbf{q}),
$$

where the subscript 0 indicates that the average is taken with the statistical weight $\exp \left(-\beta H_{0 n}\right) / Z_{0}$, with $Z_{0}$ $=\operatorname{Tr}\left[\exp \left(-\beta H_{0 n}\right)\right]$. 


\section{APPENDIX C: PERTURBATION THEORY IN THE ELASTIC NONLINEARITIES}

In this appendix, we fill in a few details on the perturbative calculation of Sec. V. We shall start by looking at the perturbative corrections to $\lambda$ and $\mu$, before addressing the relatively more involved corrections to $\kappa$ and $\Delta$.

\section{Correction to $\lambda$ and $\mu$}

Starting from Eq. (5.20) and using Wick's theorem, we find that the part of the connected average $\left\langle H_{\text {int }}^{2}\right\rangle_{0>}^{c}$, which corrects $\lambda$ and $\mu$ is given by

$$
\begin{aligned}
\left\langle H_{i n t}^{2}\right\rangle_{0>}^{c}[\lambda, \mu] \equiv & \sum_{a, b} \int d \mathbf{r} d \mathbf{r}^{\prime}\left[\mu^{2} \partial_{\alpha} u_{\beta}^{a}(\mathbf{r}) \partial_{\gamma} u_{\delta}^{b}\left(\mathbf{r}^{\prime}\right)\right. \\
& \times\left\langle\partial_{z} u_{\alpha}^{a}(\mathbf{r}) \partial_{z} u_{\beta}^{a}(\mathbf{r}) \partial_{z} u_{\gamma}^{b}\left(\mathbf{r}^{\prime}\right) \partial_{z} u_{\delta}^{b}\left(\mathbf{r}^{\prime}\right)\right\rangle_{0>}^{c} \\
& +\frac{\mu \lambda}{2} \partial_{\alpha} u_{\beta}^{a}(\mathbf{r}) \partial_{\gamma} u_{\gamma}^{b}\left(\mathbf{r}^{\prime}\right)\left\langle\partial_{z} u_{\alpha}^{a}(\mathbf{r}) \partial_{z} u_{\beta}^{a}(\mathbf{r})\right. \\
& \left.\times\left(\partial_{z} u_{\delta}^{b}\left(\mathbf{r}^{\prime}\right)\right)^{2}\right\rangle_{0>}^{c}+\frac{\lambda \mu}{2} \partial_{\alpha} u_{\alpha}^{a}(\mathbf{r}) \partial_{\gamma} u_{\delta}^{b}\left(\mathbf{r}^{\prime}\right) \\
& \times\left\langle\left[\partial_{z} u_{\beta}^{a}(\mathbf{r})\right]^{2} \partial_{z} u_{\gamma}^{b}\left(\mathbf{r}^{\prime}\right) \partial_{z} u_{\delta}^{b}\left(\mathbf{r}^{\prime}\right)\right\rangle_{0>}^{c} \\
& +\frac{\lambda^{2}}{4} \partial_{\alpha} u_{\alpha}^{a}(\mathbf{r}) \partial_{\gamma} u_{\gamma}^{b}\left(\mathbf{r}^{\prime}\right) \\
& \left.\times\left\langle\left[\partial_{z} u_{\beta}^{a}(\mathbf{r})\right]^{2}\left[\partial_{z} u_{\delta}^{b}\left(\mathbf{r}^{\prime}\right)\right]^{2}\right\rangle_{0>}^{c}\right],
\end{aligned}
$$

where we have used the symbol $\equiv$ to emphasize the fact that we only show the part that corrects $\lambda$ and $\mu$. Evaluating the above averages using Wick's theorem, we find

$$
-\frac{1}{2 T}\left\langle H_{i n t}^{2}\right\rangle_{0>}^{c}=\frac{1}{2} \int_{\mathbf{q}}^{<} u_{i}^{a<}(\mathbf{q}) \delta \Gamma_{i j}^{a b}(\mathbf{q}) u_{j}^{b<}(-\mathbf{q}),
$$

with the kernel

$$
\begin{aligned}
\delta \Gamma_{i j}^{a b}\left(\mathbf{q}_{\perp}\right)= & -\frac{1}{T} \int_{\mathbf{q}^{\prime}}^{>} q_{z}^{\prime 4}\left\{\frac{\lambda^{2}}{2} q_{i} q_{j} G_{\beta \delta}^{a b}\left(\mathbf{q}^{\prime}\right) G_{\beta \delta}^{a b}\left(\mathbf{q}+\mathbf{q}^{\prime}\right)+\mu^{2} q_{\alpha} q_{\gamma}\right. \\
& \times\left[G_{\alpha \gamma}^{a b}\left(\mathbf{q}^{\prime}\right) G_{i j}^{a b}\left(\mathbf{q}+\mathbf{q}^{\prime}\right)+G_{\alpha j}^{a b}\left(\mathbf{q}^{\prime}\right) G_{i \gamma}^{a b}\left(\mathbf{q}+\mathbf{q}^{\prime}\right)\right] \\
& +\mu \lambda q_{i} q_{\gamma} G_{\beta j}^{a b}\left(\mathbf{q}^{\prime}\right) G_{\beta \gamma}^{a b}\left(\mathbf{q}+\mathbf{q}^{\prime}\right) \\
& \left.+\lambda \mu q_{\alpha} q_{j} G_{\alpha \delta}^{a b}\left(\mathbf{q}^{\prime}\right) G_{i \delta}^{a b}\left(\mathbf{q}+\mathbf{q}^{\prime}\right)\right\}, \\
= & -\frac{1}{T} \int_{\mathbf{q}^{\prime}} q_{z}^{\prime 4}\left\{\frac{\lambda^{2}}{2} q_{i} q_{j} G_{\beta \delta}^{a b}\left(\mathbf{q}^{\prime}\right) G_{\beta \delta}^{a b}\left(\mathbf{q}^{\prime}\right)+\mu^{2} q_{\alpha} q_{\gamma}\right. \\
& \times\left[G_{\alpha \gamma}^{a b}\left(\mathbf{q}^{\prime}\right) G_{i j}^{a b}\left(\mathbf{q}^{\prime}\right)+G_{\alpha j}^{a b}\left(\mathbf{q}^{\prime}\right) G_{i \gamma}^{a b}\left(\mathbf{q}^{\prime}\right)\right] \\
& +\mu \lambda q_{i} q_{\gamma} G_{\beta j}^{a b}\left(\mathbf{q}^{\prime}\right) G_{\beta \gamma}^{a b}\left(\mathbf{q}^{\prime}\right) \\
& \left.+\lambda \mu q_{\alpha} q_{j} G_{\alpha \delta}^{a b}\left(\mathbf{q}^{\prime}\right) G_{i \delta}^{a b}\left(\mathbf{q}^{\prime}\right)\right\},
\end{aligned}
$$

where $G_{\alpha \beta}^{a b}(\mathbf{q})$ is the propagator of the theory defined by the Hamiltonian $\widetilde{H}_{0 n}$ of Eq. (5.12), which can be written in terms of transverse and longitudinal parts in the following manner:

$$
G_{\alpha \beta}^{a b}(\mathbf{q})=G_{L}^{a b}(\mathbf{q}) P_{\alpha \beta}^{L}\left(\mathbf{q}_{\perp}\right)+G_{T}^{a b}(\mathbf{q}) P_{\alpha \beta}^{T}\left(\mathbf{q}_{\perp}\right),
$$

where (in the $n \rightarrow 0$ limit)

$$
\begin{aligned}
& G_{L}^{a b}(\mathbf{q})=T \Gamma_{L}^{-1}(\mathbf{q}) \delta_{a b}+\Delta_{t} q_{z}^{2} \Gamma_{L}^{-2}(\mathbf{q}), \\
& G_{T}^{a b}(\mathbf{q})=T \Gamma_{T}^{-1}(\mathbf{q}) \delta_{a b}+\Delta_{t} q_{z}^{2} \Gamma_{T}^{-2}(\mathbf{q}) .
\end{aligned}
$$

In going from Eq. (C3) to (C3), we approximated $G_{\rho \sigma}^{a b}(\mathbf{q}$ $\left.+\mathbf{q}^{\prime}\right) \simeq G_{\rho \sigma}^{a b}\left(\mathbf{q}^{\prime}\right)$, since we only need terms of order $q_{\perp}^{2}$ in $\delta \Gamma_{i j}^{a b}\left(\mathbf{q}_{\perp}\right)$. Now, it is easy to verify, using the algebra of projection operators [Eqs. (B8a)-(B8c)], that

$$
\begin{gathered}
G_{\beta \delta}^{a b}\left(\mathbf{q}^{\prime}\right) G_{\beta \delta}^{a b}\left(\mathbf{q}^{\prime}\right)=\left[G_{L}^{a b}\left(\mathbf{q}^{\prime}\right)\right]^{2}+\left(d_{\perp}-1\right)\left[G_{T}^{a b}\left(\mathbf{q}^{\prime}\right)\right]^{2}, \\
G_{\beta j}^{a b}\left(\mathbf{q}^{\prime}\right) G_{\beta \gamma}^{a b}\left(\mathbf{q}^{\prime}\right)=\left[G_{L}^{a b}\left(\mathbf{q}^{\prime}\right)\right]^{2} P_{j \gamma}^{L}\left(\mathbf{q}_{\perp}^{\prime}\right)+\left[G_{T}^{a b}\left(\mathbf{q}^{\prime}\right)\right]^{2} P_{j \gamma}^{T}\left(\mathbf{q}_{\perp}^{\prime}\right), \\
G_{\alpha \delta}^{a b}\left(\mathbf{q}^{\prime}\right) G_{i \delta}^{a b}\left(\mathbf{q}^{\prime}\right)=\left[G_{L}^{a b}\left(\mathbf{q}^{\prime}\right)\right]^{2} P_{i \alpha}^{L}\left(\mathbf{q}_{\perp}^{\prime}\right)+\left[G_{T}^{a b}\left(\mathbf{q}^{\prime}\right)\right]^{2} P_{i \alpha}^{T}\left(\mathbf{q}_{\perp}^{\prime}\right) .
\end{gathered}
$$

The expression of $\delta \Gamma_{i j}^{a b}\left(\mathbf{q}_{\perp}\right)$, therefore, becomes

$$
\begin{aligned}
\delta \Gamma_{i j}^{a b}\left(\mathbf{q}_{\perp}\right)= & -\frac{1}{T} \int_{\mathbf{q}^{\prime}} q_{z}^{\prime 4}\left\{q_{i} q_{j}\left(\frac{\lambda^{2}}{2}+\frac{2 \lambda \mu}{d_{\perp}}\right)\right. \\
& \times\left[\left[G_{L}^{a b}\left(\mathbf{q}^{\prime}\right)\right]^{2}+\left(d_{\perp}-1\right)\left[G_{T}^{a b}\left(\mathbf{q}^{\prime}\right)\right]^{2}\right] \\
& \left.+\mu^{2} q_{\alpha} q_{\gamma}\left[G_{\alpha \gamma}^{a b}\left(\mathbf{q}^{\prime}\right) G_{i j}^{a b}\left(\mathbf{q}^{\prime}\right)+G_{\alpha j}^{a b}\left(\mathbf{q}^{\prime}\right) G_{i \gamma}^{a b}\left(\mathbf{q}^{\prime}\right)\right]\right\},
\end{aligned}
$$

where we used the following rotational averages of single projection operators (Appendix D):

$$
\left\langle P_{\alpha \beta}^{L}\left(\mathbf{q}_{\perp}\right)\right\rangle=\frac{\delta_{\alpha \beta}}{d_{\perp}},
$$

$$
\left\langle P_{\alpha \beta}^{T}\left(\mathbf{q}_{\perp}\right)\right\rangle=\frac{d_{\perp}-1}{d_{\perp}} \delta_{\alpha \beta} .
$$

Now, in the second line on the right-hand side of Eq. (C7), using the decomposition (C4), we obtain

$$
\begin{aligned}
G_{\alpha \gamma}^{a b}\left(\mathbf{q}^{\prime}\right) G_{i j}^{a b}\left(\mathbf{q}^{\prime}\right)= & {\left[G_{L}^{a b}\left(\mathbf{q}^{\prime}\right)\right]^{2} P_{\alpha \gamma}^{L}\left(\mathbf{q}_{\perp}^{\prime}\right) P_{i j}^{L}\left(\mathbf{q}_{\perp}^{\prime}\right) } \\
& +\left[G_{L}^{a b}\left(\mathbf{q}^{\prime}\right)\right]^{2} P_{\alpha \gamma}^{L}\left(\mathbf{q}_{\perp}^{\prime}\right) P_{i j}^{L}\left(\mathbf{q}_{\perp}^{\prime}\right) \\
& +G_{L}^{a b}\left(\mathbf{q}^{\prime}\right) G_{T}^{a b}\left(\mathbf{q}^{\prime}\right)\left[P_{\alpha \gamma}^{L}\left(\mathbf{q}_{\perp}^{\prime}\right) P_{i j}^{T}\left(\mathbf{q}_{\perp}^{\prime}\right)\right. \\
& \left.+P_{\alpha \gamma}^{T}\left(\mathbf{q}_{\perp}^{\prime}\right) P_{i j}^{L}\left(\mathbf{q}_{\perp}^{\prime}\right)\right] .
\end{aligned}
$$

Performing the integration over polar angles, we obtain

$$
\begin{aligned}
\mu^{2} q_{\alpha} q_{\gamma} \int_{\mathbf{q}^{\prime}} q_{z}^{\prime 4}\left[G_{\alpha \gamma}^{a b}\left(\mathbf{q}^{\prime}\right) G_{i j}^{a b}\left(\mathbf{q}^{\prime}\right)+G_{\alpha j}^{a b}\left(\mathbf{q}^{\prime}\right) G_{i \gamma}^{a b}\left(\mathbf{q}^{\prime}\right)\right] \\
=\frac{\mu^{2} q_{\perp}^{2} \delta_{i j}}{d_{\perp}\left(d_{\perp}+2\right)} \int_{\mathbf{q}^{\prime}} q_{z}^{\prime 4}\left[2\left[G_{L}^{a b}\left(\mathbf{q}^{\prime}\right)\right]^{2}+\left(d_{\perp}^{2}-2\right)\left[G_{T}^{a b}\left(\mathbf{q}^{\prime}\right)\right]^{2}\right. \\
\left.\quad+2 d_{\perp} G_{L}^{a b}\left(\mathbf{q}^{\prime}\right) G_{T}^{a b}\left(\mathbf{q}^{\prime}\right)\right]
\end{aligned}
$$




$$
\begin{aligned}
& +\frac{\mu^{2} q_{i} q_{j}}{d_{\perp}\left(d_{\perp}+2\right)} \int_{\mathbf{q}^{\prime}} q_{z}^{\prime 4}\left[4\left[G_{L}^{a b}\left(\mathbf{q}^{\prime}\right)\right]^{2}+d_{\perp}^{2}\left[G_{T}^{a b}\left(\mathbf{q}^{\prime}\right)\right]^{2}\right. \\
& \left.+2\left(d_{\perp}-2\right) G_{L}^{a b}\left(\mathbf{q}^{\prime}\right) G_{T}^{a b}\left(\mathbf{q}^{\prime}\right)\right] .
\end{aligned}
$$

Now, using the fact that

$$
\begin{gathered}
q_{i} q_{j}=q_{\perp}^{2} P_{i j}^{L}\left(\mathbf{q}_{\perp}\right), \\
\delta_{i j}=P_{i j}^{L}\left(\mathbf{q}_{\perp}\right)+P_{i j}^{T}\left(\mathbf{q}_{\perp}\right),
\end{gathered}
$$

we obtain that $\delta \Gamma_{i j}^{a b}\left(\mathbf{q}_{\perp}\right)$ can be written in the form

$$
\delta \Gamma_{i j}^{a b}\left(\mathbf{q}_{\perp}\right)=\delta \Gamma_{L}^{a b}\left(\mathbf{q}_{\perp}\right) q_{\perp}^{2} P_{i j}^{L}\left(\mathbf{q}_{\perp}\right)+\delta \Gamma_{T}^{a b}\left(\mathbf{q}_{\perp}\right) q_{\perp}^{2} P_{i j}^{T}\left(\mathbf{q}_{\perp}\right),
$$

with

$$
\begin{aligned}
\delta \Gamma_{L}^{a b}\left(\mathbf{q}_{\perp}\right)= & -\frac{1}{T}\left(\frac{\lambda^{2}}{2}+\frac{2 \lambda \mu}{d_{\perp}}\right) \int_{\mathbf{q}^{\prime}} q_{z}^{\prime 4}\left\{\left[G_{L}^{a b}\left(\mathbf{q}^{\prime}\right)\right]^{2}+\left(d_{\perp}-1\right)\right. \\
& \left.\times\left[G_{T}^{a b}\left(\mathbf{q}^{\prime}\right)\right]^{2}\right\} \pm \frac{\mu^{2}}{T d_{\perp}\left(d_{\perp}+2\right)} \int_{\mathbf{q}^{\prime}} q_{z}^{\prime 4}\left\{6\left[G_{L}^{a b}\left(\mathbf{q}^{\prime}\right)\right]^{2}\right. \\
& +\left(2 d_{\perp}^{2}-2\right)\left[G_{T}^{a b}\left(\mathbf{q}^{\prime}\right)\right]^{2}+4\left(d_{\perp}-1\right) \\
& \left.\times G_{L}^{a b}\left(\mathbf{q}^{\prime}\right) G_{T}^{a b}\left(\mathbf{q}^{\prime}\right)\right\}, \\
\delta \Gamma_{T}^{a b}\left(\mathbf{q}_{\perp}\right)= & -\frac{\mu^{2}}{T d_{\perp}\left(d_{\perp}+2\right)} \int_{\mathbf{q}^{\prime}} q_{z}^{\prime 4}\left\{2\left[G_{L}^{a b}\left(\mathbf{q}^{\prime}\right)\right]^{2}+\left(d_{\perp}^{2}-2\right)\right. \\
& \left.\times\left[G_{T}^{a b}\left(\mathbf{q}^{\prime}\right)\right]^{2}+2 d_{\perp} G_{L}^{a b}\left(\mathbf{q}^{\prime}\right) G_{T}^{a b}\left(\mathbf{q}^{\prime}\right)\right\} . \quad(\mathrm{C} 11 \mathrm{~b})
\end{aligned}
$$

Now, $\delta \Gamma_{T}^{a b}\left(\mathbf{q}_{\perp}\right)$ is nothing, but the correction to the transverse part of the elastic tensor $B_{T}$, i.e., $\delta \Gamma_{T}^{a b}\left(\mathbf{q}_{\perp}\right)=\delta \mu$. On the other hand, $\delta \Gamma_{L}^{a b}\left(\mathbf{q}_{\perp}\right)$ is the correction to the longitudinal part $B_{L}=\lambda+2 \mu$. Hence,

$$
\delta \lambda=\delta(\lambda+2 \mu)-2 \delta \mu=\delta \Gamma_{L}^{a b}\left(\mathbf{q}_{\perp}\right)-2 \delta \Gamma_{T}^{a b}\left(\mathbf{q}_{\perp}\right)
$$

Performing the above $\mathbf{q}$ integrations, we find that both corrections $\delta \mu$ and $\delta \lambda$ behave like

$$
\delta \mu \sim \delta \lambda \sim \mu^{(3-d) / 2} \kappa^{(d-7) / 2} \Delta_{t} L_{z}^{7-2 d},
$$

where $L_{z}$ is the size of the system along the direction of the flux lines (which comes in as a result of imposing an infrared cutoff $1 / L_{z}$ on $q_{z}$ integrations), which is the result (5.22) quoted in the text.

In the RG analysis of Sec. VI, the integrals in Eqs. (C11a) and $(\mathrm{C} 11 \mathrm{~b})$ are evaluated within the momentum shell defined by Eqs. (6.1a) and (6.1b), and this leads to

$$
\begin{aligned}
& \delta D=D g F_{D}(x), \\
& \delta \mu=\mu g F_{\mu}(x),
\end{aligned}
$$

where $x \equiv \lambda / \mu$ and the functions $F_{D}$ and $F_{\mu}$ are the functions defined in Eqs. (6.7a) and (6.7b).

\section{Corrections to $\kappa$ and $\Delta_{t}$}

We now turn our attention to the calculation of the perturbative corrections to the curvature modulus $\kappa$ and to the disorder strength $\Delta_{t}$. These corrections will come from the following terms of the connected average $\left\langle H_{i n t, n}^{2}\right\rangle_{0>}^{c}$ :

$$
\begin{aligned}
\left\langle H_{i n t, n}^{2}\right\rangle_{0>}^{c}\left[\kappa, \Delta_{t}\right] \equiv & \sum_{a, b} \int d \mathbf{r} d \mathbf{r}^{\prime}\left\{\mu^{2} \partial_{z} u_{\alpha}^{a<}(\mathbf{r}) \partial_{z} u_{\gamma}^{b<}\left(\mathbf{r}^{\prime}\right)\left\langle\partial_{\alpha} u_{\beta}^{a>}(\mathbf{r}) \partial_{z} u_{\beta}^{a>}(\mathbf{r}) \partial_{\gamma} u_{\delta}^{b>}\left(\mathbf{r}^{\prime}\right) \partial_{z} u_{\delta}^{b>}\left(\mathbf{r}^{\prime}\right)\right\rangle_{0>}^{c}+\mu^{2} \partial_{z} u_{\alpha}^{a<}(\mathbf{r}) \partial_{z} u_{\delta}^{b<}\left(\mathbf{r}^{\prime}\right)\right. \\
& \times\left\langle\partial_{\alpha} u_{\beta}^{a>}(\mathbf{r}) \partial_{z} u_{\beta}^{a>}(\mathbf{r}) \partial_{\gamma} u_{\delta}^{b>}\left(\mathbf{r}^{\prime}\right) \partial_{z} u_{\gamma}^{b>}\left(\mathbf{r}^{\prime}\right)\right\rangle_{0>}^{c}+\mu^{2} \partial_{z} u_{\beta}^{a<}(\mathbf{r}) \partial_{z} u_{\gamma}^{b<}\left(\mathbf{r}^{\prime}\right)\left\langle\partial_{\alpha} u_{\beta}^{a>}(\mathbf{r}) \partial_{z} u_{\alpha}^{a>}(\mathbf{r}) \partial_{\gamma} u_{\delta}^{b>}\left(\mathbf{r}^{\prime}\right) \partial_{z} u_{\delta}^{b>}\left(\mathbf{r}^{\prime}\right)\right\rangle_{0>}^{c} \\
& +\mu^{2} \partial_{z} u_{\beta}^{a<}(\mathbf{r}) \partial_{z} u_{\delta}^{b<}\left(\mathbf{r}^{\prime}\right)\left\langle\partial_{\alpha} u_{\beta}^{a>}(\mathbf{r}) \partial_{z} u_{\alpha}^{a>}(\mathbf{r}) \partial_{\gamma} u_{\delta}^{b>}\left(\mathbf{r}^{\prime}\right) \partial_{z} u_{\gamma}^{b>}\left(\mathbf{r}^{\prime}\right)\right\rangle_{0>}^{c}+\mu \lambda \partial_{z} u_{\alpha}^{a<}(\mathbf{r}) \partial_{z} u_{\delta}^{b<}\left(\mathbf{r}^{\prime}\right) \\
& \times\left\langle\partial_{\alpha} u_{\beta}^{a>}(\mathbf{r}) \partial_{z} u_{\beta}^{a>}(\mathbf{r}) \partial_{\gamma} u_{\gamma}^{b>}\left(\mathbf{r}^{\prime}\right) \partial_{z} u_{\delta}^{b>}\left(\mathbf{r}^{\prime}\right)\right\rangle_{0>}^{c}+\mu \lambda \partial_{z} u_{\beta}^{a<}(\mathbf{r}) \partial_{z} u_{\delta}^{b<}\left(\mathbf{r}^{\prime}\right)\left\langle\partial_{\alpha} u_{\beta}^{a>}(\mathbf{r}) \partial_{z} u_{\alpha}^{a>}(\mathbf{r}) \partial_{\gamma} u_{\gamma}^{b>}\left(\mathbf{r}^{\prime}\right) \partial_{z} u_{\delta}^{b>}\left(\mathbf{r}^{\prime}\right)\right\rangle_{0>}^{c} \\
& \left.+\lambda^{2} \partial_{z} u_{\beta}^{a<}(\mathbf{r}) \partial_{z} u_{\delta}^{b<}\left(\mathbf{r}^{\prime}\right)\left\langle\partial_{\alpha} u_{\alpha}^{a>}(\mathbf{r}) \partial_{z} u_{\beta}^{a>}(\mathbf{r}) \partial_{\gamma} u_{\gamma}^{b>}\left(\mathbf{r}^{\prime}\right) \partial_{z} u_{\delta}^{b>}\left(\mathbf{r}^{\prime}\right)\right\rangle_{0>}^{c}\right\}
\end{aligned}
$$

All the connected averages on the right-hand side of the above equation are of the general form $\left\langle\partial_{\alpha} u_{\beta}^{a>}(\mathbf{r}) \partial_{z} u_{\rho}^{a>}(\mathbf{r}) \partial_{\gamma} u_{\delta}^{b>}\left(\mathbf{r}^{\prime}\right) \partial_{z} u_{\sigma}^{b>}\left(\mathbf{r}^{\prime}\right)\right\rangle_{0>}^{c}$. Using Wick's theorem, we can write

$$
\begin{aligned}
& \left\langle\partial_{\alpha} u_{\beta}^{a>}(\mathbf{r}) \partial_{z} u_{\rho}^{a>}(\mathbf{r}) \partial_{\gamma} u_{\delta}^{b>}\left(\mathbf{r}^{\prime}\right) \partial_{z} u_{\sigma}^{b>}\left(\mathbf{r}^{\prime}\right)\right\rangle_{0>}^{c} \\
& =\int_{\mathbf{q}, \mathbf{q}^{\prime}}^{>}\left[q_{\alpha} q_{\gamma} q_{z}^{\prime 2} G_{\beta \delta}^{a b}(\mathbf{q}) G_{\rho \sigma}^{a b}\left(\mathbf{q}^{\prime}\right)\right. \\
& \left.\quad+q_{\alpha} q_{z} q_{z}^{\prime} q_{\gamma}^{\prime} G_{\beta \sigma}^{a b}(\mathbf{q}) G_{\rho \delta}^{a b}\left(\mathbf{q}^{\prime}\right)\right] e^{i\left(\mathbf{q}+\mathbf{q}^{\prime}\right) \cdot\left(\mathbf{r}-\mathbf{r}^{\prime}\right)} .
\end{aligned}
$$

Integration over $\mathbf{r}$ and $\mathbf{r}^{\prime}$ leads to the result

$$
\begin{array}{rl}
\int d \mathbf{r} & d \mathbf{r}^{\prime} \partial_{z} u_{\mu}^{a<}(\mathbf{r}) \partial_{z} u_{\nu}^{b<}\left(\mathbf{r}^{\prime}\right) \\
& \times\left\langle\partial_{\alpha} u_{\beta}^{a>}(\mathbf{r}) \partial_{z} u_{\rho}^{a>}(\mathbf{r}) \partial_{\gamma} u_{\delta}^{b>}\left(\mathbf{r}^{\prime}\right) \partial_{z} u_{\sigma}^{b>}\left(\mathbf{r}^{\prime}\right)\right\rangle_{0>}^{c} \\
= & \int_{\mathbf{q}}^{>} q_{z}^{2} u_{\mu}^{a<}(\mathbf{q}) u_{\nu}^{b<}(-\mathbf{q})
\end{array}
$$




$$
\begin{aligned}
& \times \int_{\mathbf{q}^{\prime}}^{>}\left[q_{\alpha} q_{\gamma}^{\prime}\left(q_{z}+q_{z}^{\prime}\right)^{2} G_{\beta \delta}^{a b}\left(\mathbf{q}^{\prime}\right)\right. \\
& \times G_{\rho \sigma}^{a b}\left(-\mathbf{q}-\mathbf{q}^{\prime}\right)+q_{\alpha} q_{z}^{\prime}\left(q_{z}+q_{z}^{\prime}\right)\left(q_{\gamma}+q_{\gamma}^{\prime}\right) \\
& \left.\times G_{\beta \sigma}^{a b}\left(\mathbf{q}^{\prime}\right) G_{\rho \delta}^{a b}\left(-\mathbf{q}-\mathbf{q}^{\prime}\right)\right] .
\end{aligned}
$$

Using the above general result, we calculate the seven terms appearing on the right-hand side of Eq. (C15), and then Taylor expand the result in $q_{z}$ near $q_{z}=0$. Tedious but straightforward calculations (which are best carried out using a symbolic math processor such as MATHEMATICA) lead to the result

$$
-\frac{1}{2 T}\left\langle H_{i n t}^{2}\right\rangle_{0>}=\int_{\mathbf{q}}^{>} u_{i}^{a<}(\mathbf{q}) \delta \Gamma_{i j}^{a b}(\mathbf{q}) u_{j}^{b<}(-\mathbf{q}),
$$

where $\delta \Gamma_{i j}^{a b}\left(q_{z}\right)$ is given by

$$
\delta \Gamma_{i j}^{a b}\left(q_{z}\right)=\delta \kappa q_{z}^{4} \delta_{a b}-\delta\left(\frac{\Delta_{t}}{T}\right) q_{z}^{2} \delta_{i j} .
$$

Again, it can be shown that evaluating the $\mathbf{q}^{\prime}$ integrals in Eq. (C16) with an IR cutoff $1 / L_{z}$ on $q_{z}$ integrations leads to same conclusion as in the previous paragraph, namely, that $\delta \kappa$ and $\delta\left(\Delta_{t} / T\right)$ both diverge with the system size in the fashion

$$
\delta \kappa \sim \delta\left(\Delta_{t} / T\right) \sim L_{z}^{7-2 d} .
$$

On the other hand, if the $\mathbf{q}^{\prime}$ integrals on the right-hand side of Eq. (C16) are evaluated within the momentum shell of Eqs. (6.1a) and (6.1b), then it follows that:

$$
\begin{gathered}
\delta \kappa=\kappa g F_{\kappa}(x), \\
\delta\left(\frac{\Delta_{t}}{T}\right)=\Delta_{t} g F_{\Delta_{t}}(x),
\end{gathered}
$$

where the functions $F_{\kappa}$ and $F_{\Delta_{t}}$ are the functions defined in Eqs. (6.7c) and (6.7d).

\section{APPENDIX D: ROTATIONAL AVERAGES OF PROJECTION OPERATORS}

In this appendix, for completeness we evaluate angular averages of projection operators, necessary for the RG computations in the main text and Appendix C. More explicitly, we want to express the following $d_{\perp}$-dimensional integrals:

$$
\begin{gathered}
I_{\alpha \beta}^{L}[f]=\int_{\mathbf{q}_{\perp}} f\left(q_{\perp}^{2}\right) P_{\alpha \beta}^{L}\left(\mathbf{q}_{\perp}\right), \\
I_{\alpha \beta}^{T}[f]=\int_{\mathbf{q}_{\perp}} f\left(q_{\perp}^{2}\right) P_{\alpha \beta}^{T}\left(\mathbf{q}_{\perp}\right), \\
I_{\alpha \beta \gamma \delta}^{L L}[f]=\int_{\mathbf{q}_{\perp}} f\left(q_{\perp}^{2}\right) P_{\alpha \beta}^{L}\left(\mathbf{q}_{\perp}\right) P_{\gamma \delta}^{L}\left(\mathbf{q}_{\perp}\right), \\
I_{\alpha \beta \gamma \delta}^{T T}[f]=\int_{\mathbf{q}_{\perp}} f\left(q_{\perp}^{2}\right) P_{\alpha \beta}^{T}\left(\mathbf{q}_{\perp}\right) P_{\gamma \delta}^{L}\left(\mathbf{q}_{\perp}\right),
\end{gathered}
$$

$$
I_{\alpha \beta \gamma \delta}^{L T}[f]=\int_{\mathbf{q}_{\perp}} f\left(q_{\perp}^{2}\right) P_{\alpha \beta}^{L}\left(\mathbf{q}_{\perp}\right) P_{\gamma \delta}^{T}\left(\mathbf{q}_{\perp}\right),
$$

where $f\left(q_{\perp}^{2}\right)$ is an arbitrary function of $q_{\perp}^{2}$, in terms of the spherically symmetric integral

$$
I[f]=\int_{\mathbf{q}_{\perp}} f\left(q_{\perp}^{2}\right) .
$$

We start with the integrals $I_{\alpha \beta}^{L}[f]$ and $I_{\alpha \beta}^{T}[f]$, which can easily be evaluated. We have (in what follows, $\hat{q}_{\alpha}$ denotes the quantity $\left.q_{\alpha} / q\right)$

$$
\begin{aligned}
I_{\alpha \beta}^{L}[f] & =\int_{\mathbf{q}_{\perp}} f\left(q_{\perp}^{2}\right) \hat{q}_{\alpha} \hat{q}_{\beta}, \\
& =\delta_{\alpha \beta} \int_{\mathbf{q}_{\perp}} f\left(q_{\perp}^{2}\right) \frac{q_{\alpha}^{2}}{q^{2}},
\end{aligned}
$$

where no summation is implied on the index $\alpha$ in the second line. Using the rotational symmetry of $f$, we can write

$$
\begin{aligned}
I_{\alpha \beta}^{L}[f] & =\frac{\delta_{\alpha \beta}}{d_{\perp}} \int_{\mathbf{q}_{\perp}} f\left(q_{\perp}^{2}\right) \frac{1}{q_{\perp}^{2}}\left(q_{1}^{2}+\cdots+q_{d_{\perp}}^{2}\right), \\
& =\frac{\delta_{\alpha \beta}}{d_{\perp}} \int_{\mathbf{q}_{\perp}} f\left(q_{\perp}^{2}\right),
\end{aligned}
$$

i.e.,

$$
I_{\alpha \beta}^{L}[f]=\frac{\delta_{\alpha \beta}}{d_{\perp}} I[f] .
$$

Similarly, we have for $I_{\alpha \beta}^{T}$

$$
\begin{aligned}
I_{\alpha \beta}^{T}[f] & =\int_{\mathbf{q}_{\perp}} f\left(q_{\perp}^{2}\right)\left[\delta_{\alpha \beta}-P_{\alpha \beta}^{L}\left(\mathbf{q}_{\perp}\right)\right], \\
& =\delta_{\alpha \beta}\left(1-\frac{1}{d_{\perp}}\right) \int_{\mathbf{q}_{\perp}} f\left(q_{\perp}^{2}\right),
\end{aligned}
$$

thus,

$$
I_{\alpha \beta}^{T}[f]=\frac{d_{\perp}-1}{d_{\perp}} \delta_{\alpha \beta} I[f] .
$$

We now turn our attention to the integral $I_{\alpha \beta, \gamma \delta}^{L L}[f]$. We first consider the case where the indices are all equal, i.e., we want to calculate the integral

$$
\begin{aligned}
I_{\alpha \alpha, \alpha \alpha}^{L L}[f] & =\int_{\mathbf{q}_{\perp}} \hat{q}_{\alpha}^{4} f\left(q_{\perp}^{2}\right), \\
& =\int_{\mathbf{q}_{\perp}} \frac{q_{\alpha}^{4}}{q_{\perp}^{4}} f\left(q_{\perp}^{2}\right) .
\end{aligned}
$$

Because of the rotational symmetry of the function $f$, this integral will be the same for all possible values of the index $\alpha=1, \ldots, d_{\perp}$. The decomposition of the vector $\mathbf{q}_{\perp}$ in spheri- 
cal coordinates in $d_{\perp}$ dimensions is given by

$$
\left\{\begin{array}{l}
q_{1}=q \sin \theta_{d_{\perp}-1} \sin \theta_{d_{\perp}-2} \cdots \sin \theta_{2} \cos \theta_{1} \\
q_{2}=q \sin \theta_{d_{\perp}-1} \sin \theta_{d_{\perp}-2} \cdots \sin \theta_{2} \sin \theta_{1} \\
q_{3}=q \sin \theta_{d_{\perp}-1} \sin \theta_{d_{\perp}-2} \cdots \sin \theta_{3} \cos \theta_{2} \\
q_{4}=q \sin \theta_{d_{\perp}-1} \sin \theta_{d_{\perp}-2} \cdots \sin \theta_{4} \cos \theta_{3} \\
\cdots \\
q_{d_{\perp}-1}=q \sin \theta_{d_{\perp}-1} \cos \theta_{d_{\perp}-2} \\
q_{d_{\perp}}=q \cos \theta_{d_{\perp}-1}
\end{array}\right.
$$

and hence we see that the most convenient choice of $\alpha$ for calculating the integral (D8) is $\alpha=d_{\perp}$. Taking $q_{\alpha}=q_{d_{\perp}}$ results in the following expression:

$$
I_{\alpha \alpha, \alpha \alpha}^{L L}[f]=\int \frac{d^{d_{\perp}} \mathbf{q}_{\perp}}{(2 \pi)^{d_{\perp}}} \cos ^{4} \theta_{d_{\perp}-1} f\left(q_{\perp}^{2}\right) .
$$

Using the fact that

$$
\begin{aligned}
d^{d_{\perp}} \mathbf{q}_{\perp}= & q^{d_{\perp}-1} \sin ^{d_{\perp}-2} \theta_{d_{\perp}-1} d \theta_{d_{\perp}-1} \sin ^{d_{\perp}-3} \theta_{d_{\perp}-2} d \theta_{d_{\perp}-2} \cdots \\
& \times \sin \theta_{2} d \theta_{2} d \theta_{1},
\end{aligned}
$$

(where $0 \leqslant \theta_{k}<\pi$ for $k \neq 1$, and $0 \leqslant \theta_{1} \leqslant 2 \pi$ ), we obtain

$$
\begin{aligned}
I_{\alpha \alpha, \alpha \alpha}^{L L}[f]= & \frac{1}{(2 \pi)^{d_{\perp}}} \int q_{\perp}^{d_{\perp}-1} f\left(q_{\perp}^{2}\right) d q_{\perp} \\
& \times \int_{0}^{\pi} \sin ^{d_{\perp}-2} \theta_{d_{\perp}-1} \cos ^{4} \theta_{d_{\perp}-1} d \theta_{d_{\perp}-1} \cdots \\
& \times \int_{0}^{\pi} \sin \theta_{2} d \theta_{2} \int_{0}^{2 \pi} d \theta_{1} \\
= & C_{d_{\perp}} \int_{\mathbf{q}_{\perp}} f\left(q_{\perp}^{2}\right)
\end{aligned}
$$

where

$$
C_{d_{\perp}}=\frac{\int_{0}^{\pi} \sin ^{d_{\perp}-2} \theta \cos ^{4} \theta d \theta}{\int_{0}^{\pi} \sin ^{d_{\perp}-2} \theta d \theta} .
$$

The integrals in the numerator and denominator of $C_{d_{\perp}}$ can be evaluated analytically. MATHEMATICA, for example, gives

$$
\begin{aligned}
C_{d_{\perp}}= & \frac{\Gamma\left(d_{\perp} / 2\right)}{\sqrt{\pi}}\left[-\frac{3 \sqrt{\pi}}{\Gamma\left(d_{\perp} / 2\right)}+2^{d_{\perp}+1}\left(\frac{3 \Gamma\left(3+d_{\perp} / 2\right)}{\Gamma\left(1+d_{\perp}\right)}\right.\right. \\
& \left.\left.-\frac{4 \Gamma\left(5+d_{\perp} / 2\right)}{\Gamma\left(2+d_{\perp}\right)}+\frac{2 \Gamma\left(7+d_{\perp} / 2\right)}{\Gamma\left(3+d_{\perp}\right)}\right)\right]
\end{aligned}
$$

Expanding this last expression using the following results for the $\Gamma$ function:

$$
\Gamma(1+z)=z \Gamma(z)
$$

$$
\sqrt{\pi} \Gamma(2 z)=2^{2 z-1} \Gamma(z) \Gamma\left(z+\frac{1}{2}\right)
$$

leads, after a few manipulations, to the very simple result

$$
C_{d_{\perp}}=\frac{3}{d_{\perp}\left(d_{\perp}+2\right)} \text {. }
$$

Hence,

$$
I_{\alpha \alpha, \alpha \alpha}^{L L}[f]=\frac{3}{d_{\perp}\left(d_{\perp}+2\right)} I[f] .
$$

We now consider the case where the indices $\alpha, \beta, \gamma$, and $\delta$ in $I_{\alpha \beta, \gamma \delta}^{L L}$ are not all equal. In that case, we can write

$$
\begin{aligned}
I_{\alpha \beta, \gamma \delta}^{L L}= & \int_{\mathbf{q}_{\perp}} f\left(q_{\perp}^{2}\right) P_{\alpha \beta}^{L}\left(\mathbf{q}_{\perp}\right) P_{\gamma \delta}^{L}\left(\mathbf{q}_{\perp}\right), \\
= & \int_{\mathbf{q}_{\perp}} f\left(q_{\perp}^{2}\right) \hat{q}_{\alpha} \hat{q}_{\beta} \hat{q}_{\gamma} \hat{q}_{\delta}, \\
= & \delta_{\alpha \beta} \delta_{\gamma \delta} \int_{\mathbf{q}_{\perp}} f\left(q_{\perp}^{2}\right) \hat{q}_{\alpha}^{2} \hat{q}_{\gamma}^{2} \\
& +\delta_{\alpha \gamma} \delta_{\beta \delta} \int_{\mathbf{q}_{\perp}} f\left(q_{\perp}^{2}\right) \hat{q}_{\alpha}^{2} \hat{q}_{\beta}^{2} \\
& +\delta_{\alpha \delta} \delta_{\beta \gamma} \int_{\mathbf{q}_{\perp}} f\left(q_{\perp}^{2}\right) \hat{q}_{\alpha}^{2} \hat{q}_{\beta}^{2},
\end{aligned}
$$

where the two remaining indices in each integral are distinct from each other. Let us evaluate one such integral. We have

$$
\begin{aligned}
\int_{\mathbf{q}_{\perp}} f\left(q^{2}\right) \hat{q}_{\alpha}^{2} \hat{q}_{\beta}^{2} & =\int_{\mathbf{q}_{\perp}} f\left(q_{\perp}^{2}\right) \frac{q_{\alpha}^{2} q_{\beta}^{2}}{q_{\perp}^{4}}, \\
& =\frac{1}{d_{\perp}-1} \int_{\mathbf{q}_{\perp}} \frac{1}{q_{\perp}^{4}} f\left(q_{\perp}^{2}\right) q_{\beta}^{2} \sum_{\alpha(\neq \beta)} q_{\alpha}^{2}, \\
& =\frac{1}{d_{\perp}-1} \int_{\mathbf{q}_{\perp}} \frac{q_{\beta}^{2}}{q_{\perp}^{4}} f\left(q_{\perp}^{2}\right)\left(q_{\perp}^{2}-q_{\beta}^{2}\right), \\
& =\frac{1}{d_{\perp}-1}\left[\int_{\mathbf{q}_{\perp}} \frac{q_{\beta}^{2}}{q_{\perp}^{2}} f\left(q_{\perp}^{2}\right)\right. \\
& \left.-\int_{\mathbf{q}_{\perp}} \frac{q_{\beta}^{4}}{q_{\perp}^{4}} f\left(q_{\perp}^{2}\right)\right] .
\end{aligned}
$$

But

$$
\int_{\mathbf{q}_{\perp}} f\left(q_{\perp}^{2}\right) \frac{q_{\beta}^{2}}{q_{\perp}^{2}}=\int_{\mathbf{q}_{\perp}} f\left(q_{\perp}^{2}\right) \hat{q}_{\beta}^{2}=\frac{1}{d_{\perp}} \int_{\mathbf{q}_{\perp}} f\left(q_{\perp}^{2}\right),
$$

and

$$
\int_{\mathbf{q}_{\perp}} f\left(q_{\perp}^{2}\right) \frac{q_{\beta}^{4}}{q_{\perp}^{4}}=\int_{\mathbf{q}_{\perp}} f\left(q_{\perp}^{2}\right) \hat{q}_{\beta}^{4}=C_{d_{\perp}} \int_{\mathbf{q}_{\perp}} f\left(q_{\perp}^{2}\right),
$$


so we obtain

$$
\begin{aligned}
\int_{\mathbf{q}_{\perp}} f\left(q_{\perp}^{2}\right) \hat{q}_{\alpha}^{2} \hat{q}_{\beta}^{2} & =\frac{1}{d_{\perp}-1}\left[\frac{1}{d_{\perp}}-C_{d_{\perp}}\right] \int_{\mathbf{q}_{\perp}} f\left(q_{\perp}^{2}\right), \\
& =\frac{1-d_{\perp} C_{d_{\perp}}}{d_{\perp}\left(d_{\perp}-1\right)} \int_{\mathbf{q}_{\perp}} f\left(q_{\perp}^{2}\right) .
\end{aligned}
$$

Using the result (D15) for $C_{d_{\perp}}$, we finally obtain

$$
\int_{\mathbf{q}_{\perp}} f\left(q_{\perp}^{2}\right) \hat{q}_{\alpha}^{2} \hat{q}_{\beta}^{2}=\frac{1}{d_{\perp}\left(d_{\perp}+2\right)} \int_{\mathbf{q}_{\perp}} f\left(q_{\perp}^{2}\right),
$$

and hence, Eq. (D17) gives ( $\alpha, \beta, \gamma$, and $\delta$ not all equal)

$$
I_{\alpha \beta, \gamma \delta}^{L L}=\frac{1}{d_{\perp}\left(d_{\perp}+2\right)}\left(\delta_{\alpha \beta} \delta_{\gamma \delta}+\delta_{\alpha \gamma} \delta_{\beta \delta}+\delta_{\alpha \delta} \delta_{\beta \gamma}\right) I[f] .
$$

It is not difficult to see that Eq. (D16) is a special case of Eq. (D23). Hence, the final result for $I_{\alpha \beta, \gamma \delta}^{L L}[f]$ is just Eq. (D23), which describes all possible combinations for indices.

Now, let us find $I_{\alpha \beta, \gamma \delta}^{L T}[f]$. We have

$$
\begin{aligned}
I_{\alpha \beta, \gamma \delta}^{L T}[f]= & \int_{\mathbf{q}_{\perp}} f\left(q_{\perp}^{2}\right) P_{\alpha \beta}^{L}\left(\mathbf{q}_{\perp}\right) P_{\gamma \delta}^{T}\left(\mathbf{q}_{\perp}\right), \\
= & \int_{\mathbf{q}_{\perp}} f\left(q_{\perp}^{2}\right) P_{\alpha \beta}^{L}\left(\mathbf{q}_{\perp}\right)\left[\delta_{\gamma \delta}-P_{\gamma \delta}^{L}\left(\mathbf{q}_{\perp}\right)\right], \\
= & \delta_{\gamma \delta} \int_{\mathbf{q}_{\perp}} f\left(q_{\perp}^{2}\right) P_{\alpha \beta}^{L}\left(\mathbf{q}_{\perp}\right)-I_{\alpha \beta, \gamma \delta}^{L L}, \\
= & \frac{1}{d_{\perp}} \delta_{\alpha \beta} \delta_{\gamma \delta} I[f]-\frac{1}{d_{\perp}\left(d_{\perp}+2\right)} \\
& \times\left(\delta_{\alpha \beta} \delta_{\gamma \delta}+\delta_{\alpha \gamma} \delta_{\beta \delta}+\delta_{\alpha \delta} \delta_{\beta \gamma}\right) I[f],
\end{aligned}
$$

hence,

$$
\begin{aligned}
I_{\alpha \beta, \gamma \delta}^{L T}[f]= & \left\{\frac{d_{\perp}+1}{d_{\perp}\left(d_{\perp}+2\right)} \delta_{\alpha \beta} \delta_{\gamma \delta}\right. \\
& \left.-\frac{1}{d_{\perp}\left(d_{\perp}+2\right)}\left(\delta_{\alpha \gamma} \delta_{\beta \delta}+\delta_{\alpha \delta} \delta_{\beta \gamma}\right)\right\} I[f] .
\end{aligned}
$$

The last rotational average that we need to calculate is $I_{\alpha \beta, \gamma \delta}^{T T}[f]$. We have

$$
I_{\alpha \beta, \gamma \delta}^{T T}[f]=\int_{\mathbf{q}_{\perp}} f\left(q_{\perp}^{2}\right) P_{\alpha \beta}^{T}\left(\mathbf{q}_{\perp}\right) P_{\gamma \delta}^{T}\left(\mathbf{q}_{\perp}\right),
$$

$$
\begin{aligned}
= & \int_{\mathbf{q}_{\perp}} f\left(q_{\perp}^{2}\right)\left[\delta_{\alpha \beta}-P_{\alpha \beta}^{L}\left(\mathbf{q}_{\perp}\right)\right]\left[\delta_{\gamma \delta}\right. \\
& \left.-P_{\gamma \delta}^{L}\left(\mathbf{q}_{\perp}\right)\right], \\
= & \delta_{\gamma \delta} \int_{\mathbf{q}_{\perp}} f\left(q_{\perp}^{2}\right)\left[\delta_{\alpha \beta} \delta_{\gamma \delta}-\delta_{\alpha \beta} P_{\gamma \delta}^{L}\left(\mathbf{q}_{\perp}\right)\right. \\
& \left.-\delta_{\gamma \delta} P_{\alpha \beta}^{L}\left(\mathbf{q}_{\perp}\right)+P_{\alpha \beta}^{L}\left(\mathbf{q}_{\perp}\right) P_{\gamma \delta}^{L}\left(\mathbf{q}_{\perp}\right)\right], \\
= & \delta_{\alpha \beta} \delta_{\gamma \delta} I[f]-\frac{2}{d_{\perp}} \delta_{\alpha \beta} \delta_{\gamma \delta} I[f] \\
& +\frac{1}{d_{\perp}\left(d_{\perp}+2\right)}\left(\delta_{\alpha \beta} \delta_{\gamma \delta}+\delta_{\alpha \gamma} \delta_{\beta \delta}\right. \\
& \left.+\delta_{\alpha \delta} \delta_{\beta \gamma}\right) I[f],
\end{aligned}
$$

hence,

$$
\begin{aligned}
I_{\alpha \beta, \gamma \delta}^{T T}[f]= & \left\{\frac{d_{\perp}^{2}-3}{d_{\perp}\left(d_{\perp}+2\right)} \delta_{\alpha \beta} \delta_{\gamma \delta}\right. \\
& \left.+\frac{1}{d_{\perp}\left(d_{\perp}+2\right)}\left(\delta_{\alpha \gamma} \delta_{\beta \delta}+\delta_{\alpha \delta} \delta_{\beta \gamma}\right)\right\} I[f] .
\end{aligned}
$$

${ }^{1}$ O. Fisher, A. Treyvand, R. Chevrel, and M. Sergent, Solid State Commun. 17, 721 (1975); R. N. Shelton, R. W. McCallum and H. Adrain, Phys. Lett. 56A, 213 (1976); W. A. Fertig, D. C. Johnston, L. E. DeLong, R. W. McCallum, M. B. Maple, and B. T. Matthias, Phys. Rev. Lett. 38, 987 (1977).

${ }^{2}$ C. Stassis, M. Bullock, J. Zarestky, P. Canfield, A. I. Goldman, G. Shirane, and S. M. Shapiro, Phys. Rev. B 55, R8678 (1997).

${ }^{3}$ P. Canfield et al. Phys. Today 51, 40 (1998).

${ }^{4}$ U. Yaron et al., Nature (London) 386, 236 (1996).

${ }^{5}$ P. C. Canfield, S. L. Bud'ko, and B. K. Cho, Physica C 262, 249 (1996).

${ }^{6}$ J. Tallon et al., IEEE Trans. Appl. Supercond. 9, 1696 (1999).

${ }^{7}$ S. S. Saxena et al., Nature (London) 406, 587 (2000); A. Huxley, I. Sheikin, E. Ressouche, N. Kernavanois, D. Braithwaite, R. Calemczuk, and J. Flouquet, Phys. Rev. B 63, 144519 (2001).
${ }^{8}$ C. Pfleiderer et al., Nature (London) 412, 58 (2001).

${ }^{9}$ D. Aoki et al., Nature (London) 413, 613 (2001).

${ }^{10}$ E. I. Blount and C. M. Varma, Phys. Rev. Lett. 42, 1079, 1979.

${ }^{11}$ M. Tachiki, H. Matsumoto, and H. Umezawa, Phys. Rev. B 20, 1915 (1979).

${ }^{12}$ C. G. Kuper, M. Revzen, and A. Ron, Phys. Rev. Lett. 44, 1545 (1980).

${ }^{13}$ M. Tachiki, H. Matsumoto, T. Koyama, and H. Umezawa, Solid State Commun. 34, 19 (1980).

${ }^{14}$ H. S. Greenside. E. I. Blount, and C. M. Varma, Phys. Rev. Lett. 46, 49 (1981).

${ }^{15}$ T. K. Ng and C. M. Varma, Phys. Rev. Lett. 78, 330 (1997).

${ }^{16}$ T. K. Ng and C. M. Varma, Phys. Rev. Lett. 78, 3745 (1997).

${ }^{17}$ H. Kawano-Furukawa, E. Habuta, T. Nagata, M. Nagao, H. 
Yoshizawa, N. Furukawa, H. Takeya, and K. Kadowaki, condmat/0106273 (unpublished).

${ }^{18}$ As discussed in detail in Sec. VIII, in real materials rotational invariance is explicitly broken by a crystal-field anisotropy. As we show there, this cuts off the physics discussed here at a length scale that is long if the anisotropy is weak, diverging with a vanishing crystal pinning field. This proviso is not special to our theory and is a property of any crystalline ferromagnet. The fully rotationally invariant theory might be best applicable to finely powdered ferromagnetic-superconductor samples.

${ }^{19}$ A. I. Larkin and Yu. N. Ovchinnikov, Sov. Phys. JETP 38, 854 (1974); J. Low Temp. Phys. 34, 409 (1979).

${ }^{20}$ E. H. Brandt, J. Low Temp. Phys. 26, 709 (1977); 26, 735 (1977); 28, 263 (1977); 28, 291 (1977).

${ }^{21}$ Karl Saunders, Ph.D. thesis, University of Oregon, 2001 (unpublished).

${ }^{22}$ A class of soft solids in which (as a result of symmetry-enforced strict vanishing of a harmonic elastic constant) nonlinear elastic terms are qualitatively important, leading to anomalous universal elasticity includes smectic $23,29,31$ and columnar liquid crystals, ${ }^{33,21}$ a flat phase of thermal and disordered polymerized membranes, ${ }^{24}$ and nematic elastomers. ${ }^{24-27}$

${ }^{23}$ G. Grinstein and R. A. Pelcovits, Phys. Rev. Lett. 47, 856 (1981).

${ }^{24}$ D. R. Nelson and L. Peliti, J. Phys. (France) 48, 1085 (1987); J. A. Aronovitz and T. C. Lubensky, Phys. Rev. Lett. 60, 2634 (1988); P. Le Doussal and L. Radzihovsky, ibid. 69, 1209 (1992).

${ }^{25}$ X. Xing, R. Mukhopadhyay, T. C. Lubensky, and L. Radzihovsky, Phys. Rev. E 68, 021108 (2003).

${ }^{26}$ T. C. Lubensky, R. Mukhapadhyay, L. Radzihovsky, and X. Xing, Phys. Rev. E 66, 011702 (2002).

${ }^{27}$ X. Xing and L. Radzihovsky, Phys. Rev. Lett. 90, 168301 (2003); Europhys. Lett. 61, 769 (2003); O. Stenull and T. C. Lubensky, ibid. 61, 776 (2003).

${ }^{28}$ P. G. de Gennes, Superconductivity of Metals and Alloys (Addison-Wesley, Reading, MA, 1966).

${ }^{29}$ L. Radzihovsky and J. Toner, Phys. Rev. Lett. 78, 4414 (1997); Phys. Rev. Lett. 79, 4214 (1997); Phys. Rev. B 60, 206 (1999).

${ }^{30}$ L. Radzihovsky, A. M. Ettouhami, K. Saunders, and J. Toner, Phys. Rev. Lett. 87, 027001 (2001).

${ }^{31}$ B. Jacobsen, K. Saunders, L. Radzihovsky, and J. Toner, Phys. Rev. Lett. 83, 1363 (1999).

${ }^{32}$ L. Chen and J. Toner, nlin.SI/0407066 (unpublished).

${ }^{33}$ K. Saunders, L. Radzihovsky, and J. Toner, Phys. Rev. Lett. 85, 4309 (2000).

${ }^{34}$ T. Giamarchi and P. Le Doussal, Phys. Rev. B 52, 1242 (1995).

${ }^{35}$ The Ginzburg-Landau functional [Eq. (2.1)] focuses on a uniform singlet superconducting order parameter, thereby from the start omitting the possibility of a Fulde-Ferrell-Larkin-Ovchinikov state expected to be induced at sufficiently large magnetization, corresponding to Zeeman energy on order of the quasiparticle gap, so as to make it favorable to break up a fraction of Cooper pairs into spin-polarized electrons.

${ }^{36}$ G. Blatter, M. V. Feigel'man, V. B. Geshkenbein, A. I. Larkin, and V. M. Vinokur, Rev. Mod. Phys. 66, 1125 (1994).

${ }^{37}$ T. Nattermann and S. Scheidl, Adv. Phys. 49, 607 (2000).

${ }^{38}$ J. Villain and J. F. Fernandez, Z. Phys. B: Condens. Matter 54, 416 (1986); T. Nattermann, Phys. Rev. Lett. 64, 2454 (1990); S. E. Korshunov, Phys. Rev. B 483969 (1993); S. Bogner, T. Emig, and T. Nattermann, ibid. 63, 174501 (2001).
${ }^{39}$ M. Mézard and G. Parisi, J. Phys. I 1, 809 (1991).

${ }^{40}$ The reader should be warned that this variational replica calculation is somewhat of an academic one, in the sense that it is valid only, strictly speaking, for $7 / 2<d<9 / 2$, where nonlinear elasticity and tilt disorder can be ignored. Including these will result in a significantly different and richer behavior for the relative displacement correlator $\overline{\left\langle[\mathbf{u}(\mathbf{r})-\mathbf{u}(\mathbf{0})]^{2}\right\rangle}$, as we shall explore in the rest of this paper.

${ }^{41}$ The effects of positional pinning on lengths scales longer than the Larkin length can be most carefully treated via a functional renormalization group method, ${ }^{51,34}$ which takes into account multiple minima of the random potential. However, here, for simplicity, we employ a less rigorous variational approach, ${ }^{39,34}$ which in conventional vortex lattices, leads to results in agreement with predictions of the functional renormalization group. ${ }^{34,38}$ The only qualitatively important result that we are after here is the logarithmic scaling of phonon correlation functions for purely positional disorder treated within harmonic elastic approximation. This is expected by general symmetry arguments and is, indeed, reproduced by replica variational approach that we take here.

${ }^{42}$ S. F. Edwards and P. W. Anderson, J. Phys. F: Met. Phys. 5, 965 (1975).

${ }^{43}$ One might worry that dominance by the tilt disorder does not survive in the presence of both positional and tilt disorder, and anharmonic elasticity. However, as was shown in a closely related randomly pinned smectic liquid-crystal system, ${ }^{29}$ here too, tilt disorder continues to dominate even if feedback effects of positional disorder are taken into account.

${ }^{44}$ As discussed in more detail in Refs. 26 and 45 the elasticity of a $d$-dimensional isotropic solid embedded in a $d$-dimensional isotropic environment can be formulated equally well in terms of the (Lagrangian) left Cauchy-Green tensor $v_{\alpha \beta}=\frac{1}{2}\left(\partial_{i} R_{\alpha} \partial_{i} R_{\beta}\right.$ $-\delta_{\alpha \beta}$ ) or the (more common, Lagrangian) right Cauchy-Green tensor $u_{i j}=\frac{1}{2}\left(\partial_{i} R_{\alpha} \partial_{j} R_{\alpha}-\delta_{i j}\right)$, with the former a scalar in the reference space and a rank- 2 tensor in the embedding space, and the latter a rank-2 tensor in the reference space and a scalar in the embedding space. However, phenomena in which an external field breaks spatial symmetry of the embedding space (as the pinning disorder does in the problem at hand), quite clearly can only be formulated in terms of the left Cauchy-Green strain tensor. Furthermore, the elasticity of solids with a dimension $d_{\perp}$ of the elastic displacements field $u_{\alpha}$ smaller than the reference space dimension $d$ (as, for example, in a line-vortex solid or a columnar liquid crystal phase, with $d_{\perp}=2$ and $d=3$ ) is also most easily formulated in terms of the left Cauchy-Green strain tensor $v_{\alpha \beta}$.

${ }^{45}$ X. Xing and L. Radzihovsky, Europhys. Lett. 61, 769 (2003); and unpublished.

${ }^{46}$ We stress that here replica trick is used purely as a technical diagrammatic device, which allows an efficient way of excluding annealed disorder contributions, that can be equally well (but less efficiently) done without the replica trick.

${ }^{47}$ It is easy to see that the first-order cumulant $\left\langle H_{\text {int }}\right\rangle_{0}$ does not yield any corrections to the parameters of the original Hamiltonian $H_{0 n}$. These corrections arise starting only at the second-order cumulant $-\left\langle H_{\text {int }}^{2}\right\rangle_{0\rangle}^{c} / 2 T$. For details, see Appendix C.

${ }^{48}$ K. G. Wilson and J. Kogut, Phys. Rep., Phys. Lett. 12, 75 (1974).

${ }^{49}$ The underlying rotational invariance of the spontaneous solid ensures that graphical (perturbative) corrections leave the nonlin- 
ear strain tensor $v_{\alpha \beta}$, Eq. (5.2), invariant, independent of the arbitrary choice of the rescaling exponents, $\phi$ and $\omega$. It is a matter of convenience to have the arbitrary rescaling to also preserve the form of the nonlinear strain tensor $v_{\alpha \beta}$, by choosing $\phi=2-\omega$ [Eq. (6.4)].

${ }^{50}$ J. Rudnick and D. R. Nelson, Phys. Rev. B 13, 2208 (1976).

${ }^{51}$ D. S. Fisher, Phys. Rev. Lett. 56, 1964 (1986).

${ }^{52}$ M. J. P. Gingras and D. A. Huse, Phys. Rev. B 53, 15193 (1996).

${ }^{53}$ J. M. Kosterlitz and D. J. Thouless, J. Phys. C 6, 1181 (1973); V. L. Berezinskii, Zh. Eksp. Teor. Fiz. 59, 907 (1970) [Sov. Phys. JETP 32, 493 (1971)]; B. I. Halperin and D. R. Nelson, Phys. Rev. Lett. 41, 121 (1978); D. R. Nelson and B. I. Halperin, Phys. Rev. B 19, 2457 (1979); A. P. Young, ibid. 19, 1855 (1979).

${ }^{54}$ P. E. Lammert, D. S. Rokhsar, and J. Toner, Phys. Rev. Lett. 70, 1650 (1993); Phys. Rev. E 52, 1778 (1995).

${ }^{55}$ J. Toner, Phys. Rev. B R26, 462 (1982).
${ }^{56}$ B. I. Halperin, T. Lubensky, and S. K. Ma, Phys. Rev. Lett. 32, 292 (1974); J. H. Chen, T. C Lubensky, and D. R. Nelson, Phys. Rev. B 17, 4274 (1978); B. I. Halperin and T. C. Lubensky Solid State Commun. 14, 997 (1973); L. Radzihovsky, Europhys. Lett. 29, 227 (1995).

${ }^{57}$ More precisely, $\sigma \sim H B(H) / 4 \pi=H\left(H_{0}+H\right) / 4 \pi$, where $H_{0}$ is the coercive field.

${ }^{58}$ K. Saunders, L. Radzihovsky, and J. Toner, Phys. Rev. Lett. 87, 027001 (2001).

${ }^{59}$ Because, as is usually the case with glassy systems, we expect equilibration into the orientationally ordered ground state to be slow; such equilibration will therefore require cooling under field alignment.

${ }^{60}$ S. Tewari, D. Belitz, T. R. Kirkpatrick, and J. Toner, Phys. Rev. Lett. 93, 177002 (2004).

${ }^{61}$ Handbook of Mathematical Functions, edited by M. Abramowitz and I. A. Stegun (Dover, New York, 1965). 